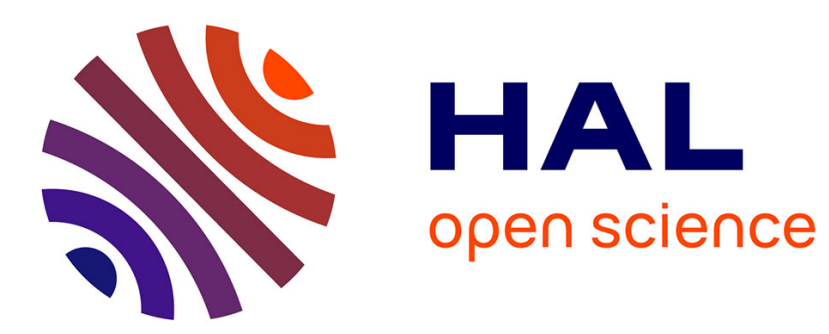

\title{
Hésitation vaccinale et corticophobie dans la dermatite atopique de l'enfant: étude observationnelle unicentrique
}

Bertille Miane

\section{To cite this version:}

Bertille Miane. Hésitation vaccinale et corticophobie dans la dermatite atopique de l'enfant: étude observationnelle unicentrique. Sciences du Vivant [q-bio]. 2020. dumas-02952003

\section{HAL Id: dumas-02952003 https://dumas.ccsd.cnrs.fr/dumas-02952003}

Submitted on 29 Sep 2020

HAL is a multi-disciplinary open access archive for the deposit and dissemination of scientific research documents, whether they are published or not. The documents may come from teaching and research institutions in France or abroad, or from public or private research centers.
L'archive ouverte pluridisciplinaire HAL, est destinée au dépôt et à la diffusion de documents scientifiques de niveau recherche, publiés ou non, émanant des établissements d'enseignement et de recherche français ou étrangers, des laboratoires publics ou privés. 
Hésitation vaccinale et corticophobie dans la dermatite atopique de l'enfant : étude observationnelle unicentrique.

\section{T H È S E}

Présentée et publiquement soutenue devant

LA FACULTÉ DES SCIENCES MEDICALES ET PARAMEDICALES

DE MARSEILLE

Le 27 Avril 2020

Par Madame Bertille MIANE

Née le 6 novembre 1990 à Marseille 08eme (13)

Pour obtenir le grade de Docteur en Médecine

D.E.S. de PÉDIATRIE

Membres du Jury de la Thèse :

Monsieur le Professeur DUBUS Jean-Christophe

Président

Madame le Professeur GAUDY-MARQUESTE

Assesseur

Caroline Monsieur le Docteur (MCU-PH) FABRE

Assesseur

Alexandre Madame le Docteur MALLET Stéphanie

Directeur

Madame le Docteur MORAND Aurélie

Assesseur 

Hésitation vaccinale et corticophobie dans la dermatite atopique de l'enfant : étude observationnelle unicentrique.

\section{T H È S E}

Présentée et publiquement soutenue devant

LA FACULTÉ DES SCIENCES MEDICALES ET PARAMEDICALES

DE MARSEILLE

Le 27 Avril 2020

Par Madame Bertille MIANE

Née le 6 novembre 1990 à Marseille 08eme (13)

Pour obtenir le grade de Docteur en Médecine

D.E.S. de PÉDIATRIE

Membres du Jury de la Thèse :

Monsieur le Professeur DUBUS Jean-Christophe

Président

Madame le Professeur GAUDY-MARQUESTE

Assesseur

Caroline Monsieur le Docteur (MCU-PH) FABRE

Assesseur

Alexandre Madame le Docteur MALLET Stéphanie

Directeur

Madame le Docteur MORAND Aurélie

Assesseur 


\section{FACULTÉ DES SCIENCES MÉDICALES \& PARAMÉDICALES}

\section{Doyen}

Vice-Doyen aux affaires générales

Vice-Doyen aux professions paramédicales

Conseiller

\section{Assesseurs :}

$>$ aux études

$>$ à la recherche

$>$ à l'unité mixte de formation continue en santé

$>$ pour le secteur NORD

$>$ Groupements Hospitaliers de territoire

$>$ aux masters
Pr. Georges LEONETTI

Pr. Patrick DESSI

Pr. Philippe BERBIS

Pr. Patrick VILLANI

Pr. Kathia CHAUMOITRE

Pr. Jean-Louis MEGE

Pr. Justin MICHEL

Pr. Stéphane BERDAH

Pr. Jean-Noël ARGENSON

Pr. Pascal ADALIAN

\section{Chargés de mission :}

sciences humaines et sociales

$>$ relations internationales

$>$ DU/DIU

$>\mathrm{DPC}$, disciplines médicales \& biologiques

$>\mathrm{DPC}$, disciplines chirurgicales
Pr. Pierre LE COZ

Pr. Stéphane RANQUE

Pr. Véronique VITTON

Pr. Frédéric CASTINETTI

Dr. Thomas GRAILLON

\section{ÉCOLE DE MEDECINE}

\section{Directeur}

\section{Chargés de mission}

- PACES - Post-PACES

- DFGSM

- DFASM

- DFASM

- Préparation aux ECN

- DES spécialités

- DES stages hospitaliers

- DES MG

- Démographie médicale

- Etudiant
Pr. Jean-Michel VITON

Pr. Régis GUIEU

Pr. Anne-Laure PELISSIER

Pr. Marie-Aleth RICHARD

Pr. Marc BARTHET

Dr Aurélie DAUMAS

Pr. Pierre-Edouard FOURNIER

Pr. Benjamin BLONDEL

Pr. Christophe BARTOLI

Dr. Noémie RESSEGUIER

Elise DOMINJON 


\section{ÉCOLE DE DE MAIEUTIQUE}

Directrice

Chargés de mission

- $1^{\mathrm{er}}$ cycle

- $2^{\text {ème }}$ cycle
Madame Carole ZAKARIAN

Madame Estelle BOISSIER

Madame Cécile NINA

\section{ÉCOLE DES SCIENCES DE LA RÉADAPTATION}

Directeur

\section{Chargés de mission}

- Masso- kinésithérapie $1^{\text {er }}$ cycle

- Masso-kinésithérapie $2^{\text {ème }}$ cycle

- Mutualisation des enseignements
Monsieur Philippe SAUVAGEON

Madame Béatrice CAORS

Madame Joannie HENRY

Madame Géraldine DEPRES

\section{ÉCOLE DES SCIENCES INFIRMIERES}

Directeur

\section{Chargés de mission}

- Chargée de mission

- Chargé de mission
Monsieur Sébastien COLSON

Madame Sandrine MAYEN RODRIGUES Monsieur Christophe ROMAN 


\section{PROFESSEURS HONORAIRES}

ATINI Serge

ALDIGHIERI René

ALESSANDRINI Pierre

ALLIEZ Bernard

AQUARON Robert

ARGEME Maxime

ASSADOURIAN Robert

AUFFRAY Jean-Pierre

AUTILLO-TOUATI Amapola

AZORIN Jean-Michel

BAILLE Yves

BARDOT Jacques

BARDOT André

BERARD Pierre

BERGOIN Maurice

BERLAND Yvon

BERNARD Dominique

BERNARD Jean-Louis

BERNARD Pierre-Marie

BERTRAND Edmond

BISSET Jean-Pierre

BLANC Bernard

BLANC Jean-Louis

BOLLINI Gérard

BONGRAND Pierre

BONNEAU Henri

BONNOIT Jean

BORY Michel

BOTTA Alain

BOURGEADE Augustin

BOUVENOT Gilles

BOUYALA Jean-Marie

BREMOND Georges

BRICOT René

BRUNET Christian

BUREAU Henri

CAMBOULIVES Jean

CANNONI Maurice

CARTOUZOU GuY

CAU Pierre

CHABOT Jean-Michel

CHAMLIAN Albert

CHARPIN Denis

CHARREL Michel

CHAUVEL Patrick

CHOUX Maurice

CIANFARANI François

CLAVERIE Jean-Michel

CLEMENT Robert

COMBALBERT André

CONTE-DEVOLX Bernard

CORRIOL Jacques

COULANGE Christian

DALMAS Henri

DE MICO Philippe

DESSEIN Alain

DELARQUE Alain

DEVIN Robert

DEVRED Philippe

DJIANE Pierre

DONNET Vincent

DUCASSOU Jacques
MM DUFOUR Michel

DUMON Henri

ENJALBERT Alain

FAVRE Roger

FIECHI Marius

FARNARIER Georges

FIGARELLA Jacques

FONTES Michel

FRANCES Yves

FRANCOIS Georges

FUENTES Pierre

GABRIEL Bernard

GALINIER Louis

GALLAIS Hervé

GAMERRE Marc

GARCIN Michel

GARNIER Jean-Marc

GAUTHIER André

GERARD Raymond

GEROLAMI-SANTANDREA André

GIUDICELLI Roger

GIUDICELLI Sébastien

GOUDARD Alain

GOUIN François

GRILLO Jean-Marie

GRISOLI François

GROULIER Pierre

HADIDA/SAYAG Jacqueline

HASSOUN Jacques

HEIM Marc

HOUEL Jean

HUGUET Jean-François

JAQUET Philippe

JAMMES Yves

JOUVE Paulette

JUHAN Claude

JUIN Pierre

KAPHAN Gérard

KASBARIAN Michel

KLEISBAUER Jean-Pierre

LACHARD Jean

LAFFARGUE Pierre

LAUGIER René

LE TREUT Yves

LEVY Samuel

LOUCHET Edmond

LOUIS René

LUCIANI Jean-Marie

MAGALON Guy

MAGNAN Jacques

MALLAN- MANCINI Josette

MALMEJAC Claude

MARANINCHI Dominique

MARTIN Claude

MATTEI Jean François

MERCIER Claude

METGE Paul

MICHOTEY Georges

MIRANDA François

MONFORT Gérard

MONGES André

MONGIN Maurice 
MM MONTIES Jean-Raoul NAZARIAN Serge

NICOLI René

NOIRCLERC Michel

OLMER Michel

OREHEK Jean

PAPY Jean-Jacques

PAULIN Raymond

PELOUX Yves

PENAUD Antony

PENE Pierre

PIANA Lucien

PICAUD Robert

PIGNOL Fernand

POGGI Louis

POITOUT Dominique

PONCET Michel

POUGET Jean

PRIVAT Yvan

QUILICHINI Francis

RANQUE Jacques

RANQUE Philippe

RICHAUD Christian

RIDINGS Bernard

ROCHAT Hervé

ROHNER Jean-Jacques

ROUX Hubert

ROUX Michel

RUFO Marcel

SAHEL José

SALAMON Georges

SALDUCCI Jacques

SAN MARCO Jean-Louis

SANKALE Marc

SARACCO Jacques

SARLES Jacques

SASTRE Bernard

SCHIANO Alain

SCOTTO Jean-Claude

SEBAHOUN Gérard

SERMENT Gérard

SOULAYROL René

STAHL André

TAMALET Jacques

TARANGER-CHARPIN Colette

THOMASSIN Jean-Marc

UNAL Daniel

VAGUE Philippe

VAGUE/JUHAN Irène
VANUXEM Paul

VERVLOET Daniel

VIALETTES Bernard

WEILLER Pierre-Jean 
2008

M. le Professeur Mme le Professeur M. le Professeur M. le Professeur M. le Professeur

\section{9}

M. le Professeur

M. le Professeur

\section{0}

M. le Professeur

2011

M. le Professeur

M. le Professeur

M. le Professeur

2012

M. le Professeur

M. le Professeur

M. le Professeur

M. le Professeur

M. le Professeur

M. le Professeur

M. le Professeur

\section{3}

M. le Professeur M. le Professeur M. le Professeur M. le Professeur M. le Professeur M. le Professeur M. le Professeur M. le Professeur

\section{4}

M. le Professeur M. le Professeur M. le Professeur M. le Professeur M. le Professeur

\section{5}

M. le Professeur M. le Professeur M. le Professeur M. le Professeur M. le Professeur M. le Professeur
LEVY Samuel

$31 / 08 / 2011$

$31 / 08 / 2011$

$31 / 08 / 2011$

$31 / 08 / 2011$

$31 / 08 / 2011$

ROBERTOUX Pierre

$31 / 08 / 2011$

$31 / 08 / 2012$

$31 / 12 / 2014$

$31 / 08 / 2015$

$31 / 08 / 2015$

$31 / 08 / 2015$

$31 / 08 / 2015$

$31 / 08 / 2015$

$31 / 08 / 2015$

$31 / 08 / 2015$

$31 / 08 / 2015$

$31 / 08 / 2015$

$31 / 08 / 2015$

$31 / 08 / 2016$

$31 / 08 / 2016$

$31 / 08 / 2016$

$31 / 08 / 2016$

$31 / 08 / 2016$

$31 / 08 / 2016$

$31 / 08 / 2016$

$31 / 08 / 2016$

$31 / 08 / 2017$

$31 / 08 / 2017$

$31 / 08 / 2017$

$31 / 08 / 2017$

$31 / 08 / 2017$

$31 / 08 / 2018$

$31 / 08 / 2018$

$31 / 08 / 2016$

$31 / 08 / 2016$

$31 / 08 / 2016$

$31 / 08 / 2016$ 
EMERITAT

2016

M. le Professeur M. le Professeur M. le Professeur M. le Professeur M. le Professeur M. le Professeur M. le Professeur M. le Professeur M. le Professeur M. le Professeur M. le Professeur M. le Professeur M. le Professeur 2017

M. le Professeur M. le Professeur M. le Professeur M. le Professeur M. le Professeur M. le Professeur M. le Professeur M. le Professeur

\section{8}

M. le Professeur M. le Professeur M. le Professeur M. le Professeur M. le Professeur M. le Professeur

2019

M. le Professeur M. le Professeur M. le Professeur M. le Professeur M. le Professeur M. le Professeur M. le Professeur M. le Professeur M. le Professeur M. le Professeur M. le Professeur M. le Professeur M. le Professeur
BONGRAND Pierre BOUVENOT Gilles BRUNET Christian

CAU Pierre

COZZONE Patrick

FAVRE Roger

FONTES Michel

JAMMES Yves

NAZARIAN Serge

OLIVER Charles

POITOUT Dominique

SEBAHOUN Gérard

VIALETTES Bernard

ALESSANDRINI Pierre

$31 / 08 / 2020$

$31 / 08 / 2018$

$31 / 08 / 2020$

$31 / 08 / 2018$

$31 / 08 / 2018$

$31 / 08 / 2018$

$31 / 08 / 2018$

$31 / 08 / 2018$

MARANINCHI Dominique

$31 / 08 / 2021$

$31 / 08 / 2019$

$31 / 08 / 2019$

$31 / 08 / 2019$

$31 / 08 / 2019$

$31 / 08 / 2019$

$31 / 08 / 2022$

$31 / 08 / 2022$

$31 / 08 / 2022$

$31 / 08 / 2022$

$31 / 08 / 2020$

$31 / 08 / 2020$

$31 / 08 / 2020$

$31 / 08 / 2020$

$31 / 08 / 2020$

$31 / 08 / 2020$

$31 / 08 / 2020$

$31 / 08 / 2020$

$31 / 08 / 2020$ 


\section{PROFESSEURS DES UNIVERSITES-PRATICIENS HOSPITALIERS}

AGOSTINI FERRANDES Aubert

ALBANESE Jacques

ALIMI Yves

AMABILE Philippe

AMBROSI Pierre

ANDRE Nicolas

ARGENSON Jean-Noël

ASTOUL Philippe

ATTARIAN Shahram

AUDOUIN Bertrand

AUQUIER Pascal

AVIERINOS Jean-François

AZULAY Jean-Philippe

BAILLY Daniel

BARLESI Fabrice

BARLIER-SETTI Anne

BARTHET Marc

BARTOLI Christophe

BARTOLI Jean-Michel

BARTOLI Michel

BARTOLOMEI Fabrice

BASTIDE Cyrille

BENSOUSSAN Laurent

BERBIS Philippe

BERBIS Julie

BERDAH Stéphane

BERNARD Jean-Paul Retraite au 25/11/2019

BEROUD Christophe

BERTUCCI François

BLAISE Didier

BLIN Olivier

BLONDEL Benjamin

BONIN/GUILLAUME Sylvie

BONELLO Laurent

BONNET Jean-Louis

BOTTA/FRIDLUND Danielle Surnombre

BOUBLI Léon Surnombre

BOUFI Mourad

BOYER Laurent

BREGEON Fabienne

BRETELLE Florence

BROUQUI Philippe

BRUDER Nicolas

BRUE Thierry

BRUNET Philippe

BURTEY Stéphane

CARCOPINO-TUSOLI Xavier

CASANOVA Dominique

CASTINETTI Frédéric

CECCALDI Mathieu

CHAGNAUD Christophe

CHAMBOST Hervé

CHAMPSAUR Pierre

CHANEZ Pascal

CHARAFFE-JAUFFRET Emmanuelle

CHARREL Rémi

CHAUMOITRE Kathia

CHIARONI Jacques

CHINOT Olivier
CHOSSEGROS Cyrille

COLLART Frédéric

COSTELLO Régis

COURBIERE Blandine

COWEN Didier

CRAVELLO Ludovic

CUISSET Thomas

CURVALE Georges Surnombre

DA FONSECA David

DAHAN-ALCARAZ Laetitia

DANIEL Laurent

DARMON Patrice

D'ERCOLE Claude

D'JOURNO Xavier

DEHARO Jean-Claude

DELAPORTE Emmanuel

DELPERO Jean-Robert Surnombre

DENIS Danièle

DISDIER Patrick

DODDOLI Christophe

DRANCOURT Michel

DUBUS Jean-Christophe

DUFFAUD Florence

DUFOUR Henry

DURAND Jean-Marc

DUSSOL Bertrand

EBBO Mikaël

EUSEBIO Alexandre

FAKHRY Nicolas

FAUGERE Gérard Surnombre

FELICIAN Olvier

FENOLLAR Florence

FIGARELLA/BRANGER Dominique

FLECHER Xavier

FOURNIER Pierre-Edouard

FRANCESCHI Frédéric

FUENTES Stéphane

GABERT Jean

GABORIT Bénédicte

GAINNIER Marc

GARCIA Stéphane

GARIBOLDI Vlad

GAUDART Jean

GAUDY-MARQUESTE Caroline

GENTILE Stéphanie

GERBEAUX Patrick

GEROLAMI/SANTANDREA René

GILBERT/ALESSI Marie-Christine

GIORGI Roch

GIOVANNI Antoine

GIRARD Nadine

GIRAUD/CHABROL Brigitte

GONCALVES Anthony

GRANEL/REY Brigitte

GRANVAL Philippe

GREILLIER Laurent

GRIMAUD Jean-Charles

GROB Jean-Jacques
GUEDJ Eric

GUIEU Régis

GUIS Sandrine

GUYE Maxime

GUYOT Laurent

GUYS Jean-Michel Surnombre

HABIB Gilbert

HARDWIGSEN Jean

HARLE Jean-Robert

HOFFART Louis Disponibilité

HOUVENAEGHEL Gilles

JACQUIER Alexis

JOURDE-CHICHE Noémie

JOUVE Jean-Luc

KAPLANSKI Gilles

KARSENTY Gilles

KERBAUL François détachement

KRAHN Martin

LAFFORGUE Pierre

LAGIER Jean-Christophe

LAMBAUDIE Eric

LANCON Christophe

LA SCOLA Bernard

LAUNAY Franck

LAVIEILLE Jean-Pierre

LE CORROLLER Thomas

LECHEVALLIER Eric

LEGRE Régis

LEHUCHER-MICHEL Marie-Pascale

LEONE Marc

LEONETTI Georges

LEPIDI Hubert

LEVY Nicolas

MACE LoÏc

MAGNAN Pierre-Edouard

MANCINI Julien

MATONTI Frédéric Disponibilité

MEGE Jean-Louis

MERROT Thierry

METZLER/GUILLEMAIN Catherine

MEYER/DUTOUR Anne

MICCALEF/ROLL Joëlle

MICHEL Fabrice

MICHEL Gérard

MICHEL Justin

MICHELET Pierre

MILH Mathieu

MILLION Matthieu

MOAL Valérie

MORANGE Pierre-Emmanuel

MOULIN GuY

MOUTARDIER Vincent

MUNDLER Olivier Surnombre

NAUDIN Jean

NICOLAS DE LAMBALLERIE Xavier

NICOLLAS Richard

OLIVE Daniel

OUAFIK L'Houcine

OVAERT-REGGIO Caroline 


\title{
PROFESSEURS DES UNIVERSITES-PRATICIENS HOSPITALIERS
}

PAGANELLI Franck

PANUEL Michel

PAPAZIAN Laurent

PAROLA Philippe

PARRATTE Sébastien Disponibilité

PELISSIER-ALICOT Anne-Laure

PELLETIER Jean

PERRIN Jeanne

PETIT Philippe

PHAM Thao

PIERCECCHI/MARTI Marie-Dominique

PIQUET Philippe

PIRRO Nicolas

POINSO François

RACCAH Denis

RANQUE Stéphane

RAOULT Didier

REGIS Jean

REYNAUD/GAUBERT Martine

REYNAUD Rachel

RICHARD/LALLEMAND Marie-Aleth

ROCHE Pierre-Hugues
ROCH Antoine

ROCHWERGER Richard

ROLL Patrice

ROSSI Dominique

ROSSI Pascal

ROUDIER Jean

SALAS Sébastien

SAMBUC Roland Surnombre

SARLES/PHILIP Nicole

SARLON-BARTOLI Gabrielle

SCAVARDA Didier

SCHLEINITZ Nicolas

SEBAG Frédéric

SEITZ Jean-François

SIELEZNEFF Igor

SIMON Nicolas

STEIN Andréas

TAIEB David

THIRION Xavier

THOMAS Pascal

THUNY Franck

TREBUCHON-DA FONSECA Agnès
TRIGLIA Jean-Michel TROPIANO Patrick TSIMARATOS Michel TURRINI Olivier

VALERO René VAROQUAUX Arthur Damien VELLY Lionel

VEY Norbert

VIDAL Vincent

VIENS Patrice

VILLANI Patrick

VITON Jean-Michel

VITTON Véronique

VIEHWEGER Heide Elke

VIVIER Eric

XERRI LUC

\section{PROFESSEUR DES UNIVERSITES}

\author{
ADALIAN Pascal \\ AGHABABIAN Valérie \\ BELIN Pascal \\ CHABANNON Christian \\ CHABRIERE Eric \\ FERON François \\ LE COZ Pierre \\ LEVASSEUR Anthony \\ RANJEVA Jean-Philippe \\ SOBOL Hagay \\ PROFESSEUR CERTIFIE
}

BRANDENBURGER Chantal

PRAG

TANTI-HARDOUIN Nicolas

PROFESSEUR DES UNIVERSITES MEDECINE GENERALE

GENTILE Gaëtan

PROFESSEUR ASSOCIE DE MEDECINE GENERALE $A$ MI-TEMPS

ADNOT Sébastien

GUIDA Pierre

PROFESSEUR ASSOCIE DES UNIVERSITES (disciplines médicales) 


\title{
MAITRES DE CONFERENCES DES UNIVERSITES-PRATICIENS HOSPITALIERS
}

AHERFI Sarah

ELDIN Carole

ANGELAKIS Emmanouil (disponibilité) FABRE Alexandre

ATLAN Catherine (disponibilité)

FAURE Alice

FOLETTI Jean- Marc

FOUILLOUX Virginie

FRANKEL Diane

FROMONOT Julien

GASTALDI Marguerite

GELSI/BOYER Véronique

GIUSIANO Bernard

GIUSIANO COURCAMBECK Sophie

GONZALEZ Jean-Michel

GOURIET Frédérique

GRAILLON Thomas

GUERIN Carole

GUENOUN MEYSSIGNAC Daphné

GUIDON Catherine

GUIVARCH Jokthan

HAUTIER/KRAHN Aurélie

HRAIECH Sami

KASPI-PEZZOLI Elise

L'OLLIVIER Coralie

LABIT-BOUVIER Corinne

LAFAGE/POCHITALOFF-HUVALE Marina

LAGIER Aude (disponibilité)

LAGOUANELLE/SIMEONI Marie-Claude

LEVY/MOZZICONACCI Annie

LOOSVELD Marie

MAAROUF Adil

MACAGNO Nicolas

MAUES DE PAULA André

MOTTOLA GHIGO Giovanna

NGUYEN PHONG Karine
NINOVE Laetitia

NOUGAIREDE Antoine

OLLIVIER Matthieu

PAULMYER/LACROIX Odile

PESENTI Sébastien

RADULESCO Thomas

RESSEGUIER Noémie

ROBERT Philippe

ROMANET Pauline

SABATIER Renaud

SARI-MINODIER Irène

SAVEANU Alexandru

SECQ Véronique (disponibilité)

STELLMANN Jan-Patrick

SUCHON Pierre

TABOURET Emeline

TOGA Caroline

TOGA Isabelle

TOMASINI Pascale

TOSELLO Barthélémy

TROUSSE Delphine

TUCHTAN-TORRENTS Lucile

VELY Frédéric

VION-DURY Jean

ZATTARA/CANNONI Hélène

MAITRES DE CONFERENCES DES UNIVERSITES

(mono-appartenants)

ABU ZAINEH Mohammad BARBACARU/PERLES T. A. BERLAND/BENHAIM Caroline BOUCAULT/GARROUSTE Françoise BOYER Sylvie COLSON Sébastien

\author{
DESNUES Benoît \\ MARANINCHI Marie \\ MERHEJ/CHAUVEAU Vicky \\ MINVIELLE/DEVICTOR Bénédicte \\ POGGI Marjorie
}

DEGIOANNI/SALLE Anna
POUGET Benoît

RUEL Jérôme

THOLLON Lionel

THIRION Sylvie

VERNA Emeline

MAITRE DE CONFERENCES DES UNIVERSITES DE MEDECINE GENERALE

CASANOVA Ludovic

MAITRES DE CONFERENCES ASSOCIES DE MEDECINE GENERALE à MI-TEMPS

\author{
BARGIER Jacques \\ BONNET Pierre-André \\ CALVET-MONTREDON Céline \\ JANCZEWSKI Aurélie \\ NUSSLI Nicolas \\ ROUSSEAU-DURAND Raphaëlle \\ THERY Didier (nomination au 1/10/2019)
}


MAITRE DE CONFERENCES ASSOCIE à MI-TEMPS

BOURRIQUEN Maryline

EVANS-VIALLAT Catherine

LUCAS Guillaume

MATHIEU Marion

MAYENS-RODRIGUES Sandrine

MELLINAS Marie

REVIS Joana

ROMAN Christophe

TRINQUET Laure 


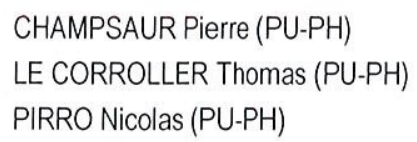

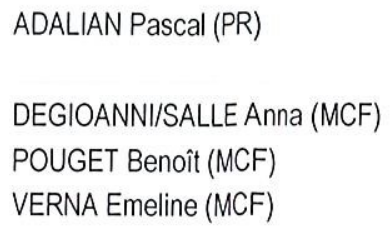

BACTERIOLOGIE-VIROLOGIE ; HYGIENE HOSPITALIERE 4501

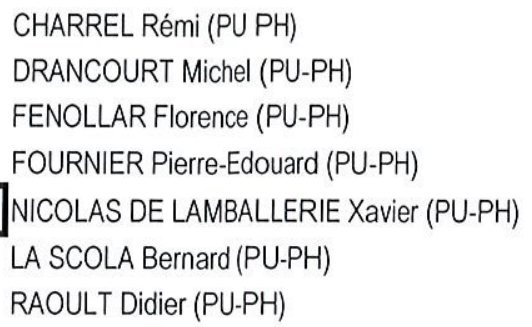

CHABRIERE Eric (PR) (64ème section)

LEVASSEUR Anthony (PR) (64ème section) DESNUES Benoit (MCF) ( 65ème section ) MERHEJ/CHAUVEAU Vicky (MCF) (87ème section)

BIOCHIMIE ET BIOLOGIE MOLECULAIRE 4401

BARLIER/SETTI Anne (PU-PH)

GABERT Jean (PU-PH)

GUIEU Régis (PU-PH)

OUAFIK L'Houcine (PU-PH)

BUFFAT Christophe (MCU-PH)

FROMONOT Julien (MCU-PH)

MOTTOLA GHIGO Giovanna (MCU-PH)

ROMANET Pauline (MCU-PH)

SAVEANU Alexandru (MCU-PH)
ROLL Patrice (PU-PH)

FRANKEL Diane (MCU-PH)

GASTALDI Marguerite (MCU-PH)

KASPI-PEZZOLI Elise (MCU-PH)

BIOLOGIE ET MEDECINE DU DEVELOPPEMENT

LEVY-MOZZICONNACCI Annie (MCU-PH) 


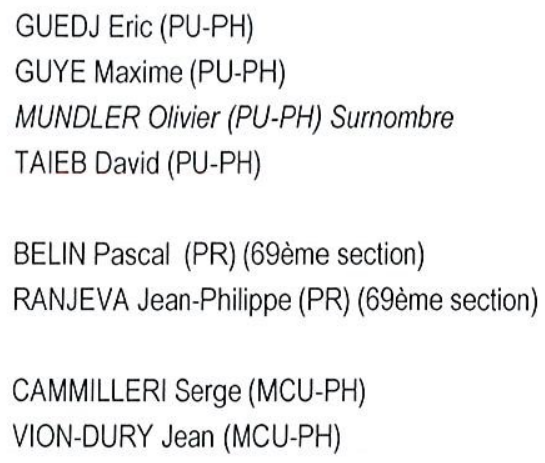

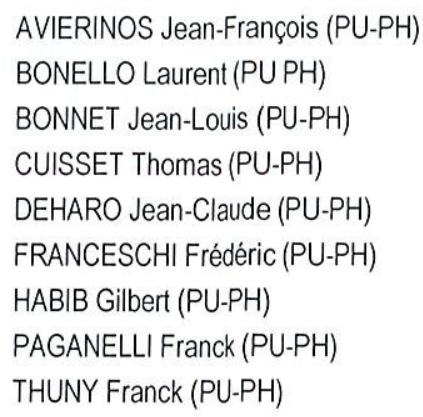

\section{CHIRURGIE ORTHOPEDIQUE ET TRAUMATOLOGIQUE 5002}

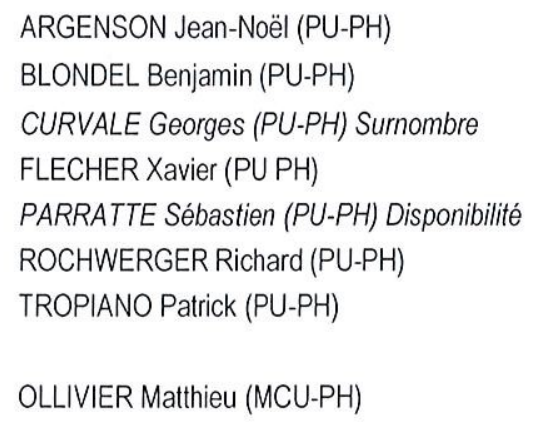

BERDAH Stéphane (PU-PH)

DELPERO Jean-Robert (PU-PH) Surnombre HARDWIGSEN Jean (PU-PH)

MOUTARDIER Vincent (PU-PH)

SEBAG Frédéric (PU-PH)

SIELEZNEFF Igor (PU-PH)

TURRINI Olivier (PU-PH)

BEGE Thierry (MCU-PH)

BEYER-BERJOT Laura (MCU-PH)

BIRNBAUM David (MCU-PH)

DUCONSEIL Pauline (MCU-PH)

GUERIN Carole (MCU PH)

\section{HIRURGIE VISCERALE ET DIGESTIVE 5202}

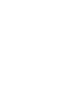




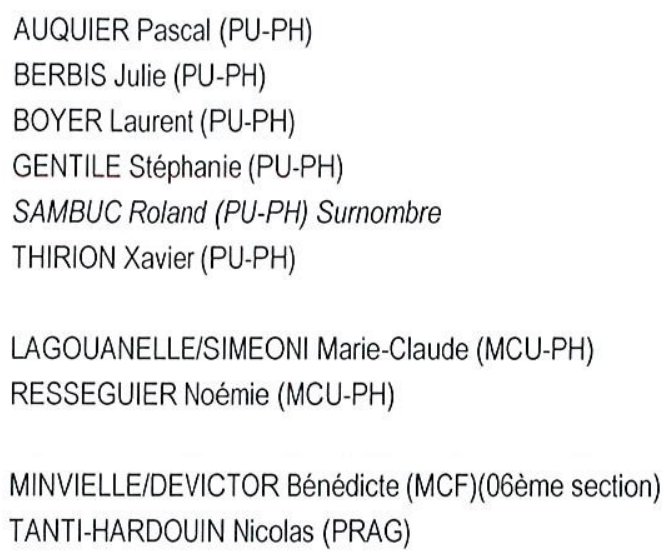

\section{MEDECINE LEGALE ET DROIT DE LA SANTE 4603}

KAPLANSKI Gilles (PU-PH)

MEGE Jean-Louis (PU-PH)

OLIVE Daniel (PU-PH)

VIVIER Eric (PU-PH)

FERON François (PR) (69ème section)

BOUCRAUT Joseph (MCU-PH)

CHRETIEN Anne-Sophie (MCU PH)

DEGEORGES/VITTE Joëlle (MCU-PH)

DESPLAT/JEGO Sophie (MCU-PH)

ROBERT Philippe (MCU-PH)

VELY Frédéric (MCU-PH)

BOUCAULT/GARROUSTE Françoise (MCF) 65ème section)

\section{BARTOLI Christophe (PU-PH)}

LEONETTI Georges (PU-PH)

PELISSIER-ALICOT Anne-Laure (PU-PH)

PIERCECCHI-MARTI Marie-Dominique (PU-PH)

TUCHTAN-TORRENTS Lucile (MCU-PH)

BERLAND/BENHAIM Caroline (MCF) (1ère section)

\section{MALADIES INFECTIEUSES ; MALADIES TROPICALES 4503}

BROUQUI Philippe (PU-PH)

LAGIER Jean-Christophe (PU-PH)

MILLION Matthieu (PU-PH)

PAROLA Philippe (PU-PH)

STEIN Andréas (PU-PH)

ELDIN Carole (MCU-PH) MEDECINE D'URGENCE 4805
MEDECINE PHYSIQUE ET DE READAPTATION 4905

BENSOUSSAN Laurent (PU-PH)

VITON Jean-Michel (PU-PH)

MEDECINE ET SANTE AU TRAVAIL 4602

LEHUCHER/MICHEL Marie-Pascale (PU-PH)

BERGE-LEFRANC Jean-Louis (MCU-PH) SARI/MINODIER Irène (MCU-PH)

KERBAUL François (PU-PH) détachement

MICHELET Pierre (PU-PH)

MEDECINE INTERNE ; GERIATRIE ET BIOLOGIE DU VIEILLISSEMENT ; ADDICTOLOGIE 5301

BONIN/GUILLAUME Sylvie (PU-PH)

DISDIER Patrick (PU-PH)

DURAND Jean-Marc (PU-PH)

GRANEL/REY Brigitte (PU-PH)

HARLE Jean-Robert (PU-PH)

ROSSI Pascal (PU-PH)

SCHLEINITZ Nicolas (PU-PH) 


\section{GENTILE Gaëtan (PR Méd. Gén. Temps plein) \\ CASANOVA Ludovic (MCF Méd. Gén. Temps plein) \\ ADNOT Sébastien (PR associé Méd. Gén. à mi-temps) \\ GUIDA Pierre (PR associé Méd. Gén. à mi-temps)}

BARGIER Jacques (MCF associé Méd. Gén. À mi-temps)

BONNET Pierre-André (MCF associé Méd. Gén à mi-temps)

CALVET-MONTREDON Céline (MCF associé Méd. Gén. à temps plein)

JANCZEWSKI Aurélie (MCF associé Méd. Gén. À mi-temps)

NUSSLI Nicolas (MCF associé Méd. Gén. À mi-temps)

ROUSSEAU-DURAND Raphaëlle (MCF associé Méd. Gén. À mi-temps)

THERY Didier (MCF associé Méd. Gén. À mi-temps) (nomination au 1/10/2019)
BRUNET Philippe (PU-PH)

BURTEY Stépahne (PU-PH)

DUSSOL Bertrand (PU-PH)

JOURDE CHICHE Noémie (PU PH)

MOAL Valérie (PU-PH)

\section{NUTRITION 4404}

\section{NEUROCHIRURGIE 4902}

DUFOUR Henry (PU-PH)

FUENTES Stéphane (PU-PH)

REGIS Jean (PU-PH)

ROCHE Pierre-Hugues (PU-PH)

SCAVARDA Didier (PU-PH)

CARRON Romain (MCU PH)

GRAILLON Thomas (MCU PH)

DARMON Patrice (PU-PH)

RACCAH Denis (PU-PH)

VALERO René (PU-PH)

\section{ONCOLOGIE 65 (BIOLOGIE CELLULAIRE)}

CHABANNON Christian (PR) (66ème section)

SOBOL Hagay (PR) (65ème section)

\section{OPHTALMOLOGIE 5502}

DENIS Danièle (PU-PH)

HOFFART Louis (PU-PH) Disponibilité

MATONTI Frédéric (PU-PH) Disponibilité
ATTARIAN Sharham (PUPH)

AUDOIN Bertrand (PU-PH)

AZULAY Jean-Philippe (PU-PH)

CECCALDI Mathieu (PU-PH)

EUSEBIO Alexandre (PU-PH)

FELICIAN Olivier (PU-PH)

PELLETIER Jean (PU-PH)

MAAROUF Adil (MCU-PH)

PEDOPSYCHIATRIE; ADDICTOLOGIE 4904

\section{DA FONSECA David (PU-PH)}

POINSO François (PU-PH)

\section{OTO-RHINO-LARYNGOLOGIE 5501}

DESSI Patrick (PU-PH)

FAKHRY Nicolas (PU-PH)

GUIVARCH Jokthan (MCU-PH)

GIOVANNI Antoine (PU-PH)

LAVIEILLE Jean-Pierre (PU-PH)

MICHEL Justin (PU-PH)

NICOLLAS Richard (PU-PH)

TRIGLIA Jean-Michel (PU-PH)

RADULESCO Thomas (MCU-PH)

REVIS Joana (MAST) (Orthophonie) (7ème Section)
PHARMACOLOGIE FONDAMENTALE -

PHARMACOLOGIE CLINIQUE; ADDICTOLOGIE 4803

\author{
BLIN Olivier (PU-PH) \\ FAUGERE Gérard (PU-PH) Surnombre \\ MICALLEF/ROLL Joëlle (PU-PH) \\ SIMON Nicolas (PU-PH)
}

BOULAMERY Audrey (MCU-PH) 


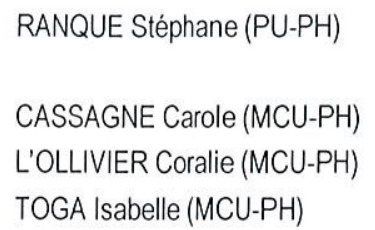

\section{PHYSIOLOGIE 4402}

BARTOLOMEI Fabrice (PU-PH)

BREGEON Fabienne (PU-PH)

GABORIT Bénédicte (PU-PH)

MEYER/DUTOUR Anne (PU-PH)

TREBUCHON/DA FONSECA Agnès (PU-PH)

BARTHELEMY Pierre (MCU-PH)

BONINI Francesca (MCU-PH)

BOULLU/CIOCCA Sandrine (MCU-PH)

DADOUN Frédéric (MCU-PH) (disponibilité)

DELLIAUX Stéphane (MCU-PH)

\section{PSYCHIATRIE D'ADULTES ; ADDICTOLOGIE 4903}

BAILLY Daniel (PU-PH)

LANCON Christophe (PU-PH)

NAUDIN Jean (PU-PH)

PNEUMOLOGIE; ADDICTOLOGIE 5101

CERMOLACCE Michel (MCU-PH)

ASTOUL Philippe (PU-PH)

BARLESI Fabrice (PU-PH)

CHANEZ Pascal (PU-PH)

GREILLIER Laurent (PU PH)

PSYCHOLOGIE - PSYCHOLOGIE CLINIQUE, PCYCHOLOGIE SOCIALE 16

REYNAUD/GAUBERT Martine (PU-PH)

AGHABABIAN Valérie (PR)

TOMASINI Pascale (MCU-PH)

\section{RADIOLOGIE ET IMAGERIE MEDICALE 4302}

\section{RHUMATOLOGIE 5001}

BARTOLI Jean-Michel (PU-PH)

CHAGNAUD Christophe (PU-PH)

CHAUMOITRE Kathia (PU-PH)

GIRARD Nadine (PU-PH)

JACQUIER Alexis (PU-PH)

MOULIN GUY (PU-PH)

PANUEL Michel (PU-PH)

PETIT Philippe (PU-PH)

VAROQUAUX Arthur Damien (PU-PH)

VIDAL Vincent (PU-PH)
GUIS Sandrine (PU-PH) LAFFORGUE Pierre (PU-PH) PHAM Thao (PU-PH) ROUDIER Jean (PU-PH)

THERAPEUTIQUE; MEDECINE D'URGENCE; ADDICTOLOGIE 4804

AMBROSI Pierre (PU-PH) VILLANI Patrick (PU-PH)

DAUMAS Aurélie (MCU-PH)

REANIMATION MEDICALE ; MEDECINE URGENCE 4802

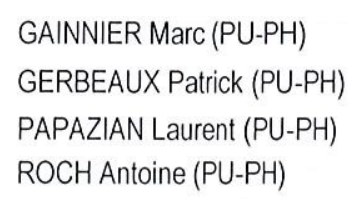

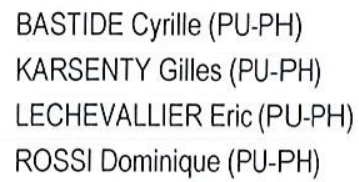




\section{REMERCIEMENTS}

\section{A mon jury de thèse,}

A Monsieur le Pr Dubus, merci de m'avoir fait l'honneur de présider ce jury de thèse. Je vous remercie pour votre patience, votre gentillesse et disponibilité tout au long de mon parcours. Veuillez trouver l'expression de ma plus grande gratitude.

A Madame le Dr Mallet, merci d'avoir accepté de diriger ma thèse.

Merci de t'être si impliquée, de m'avoir épaulée, comprise et motivée pendant ce travail jusqu'au dernier moment. Je te remercie de m'avoir si agréablement transmis ta passion pour la dermato pédiatrie. Travailler à tes côtés avec joie et bonne humeur a été un réel plaisir.

A Madame le Pr Gaudy, merci de m'avoir fait l'honneur d'accepter d'être membre de mon jury de thèse.

Je vous remercie de m'avoir accueillie chaleureusement au sein de votre équipe ce semestre et de l'intérêt que vous portez à mon travail. Soyez assurée de ma profonde gratitude.

A Monsieur le Dr Fabre, merci de m'avoir fait l'honneur d'accepter d'être membre de mon jury de thèse.

Merci pour ton enseignement, ton écoute, ta simplicité et tes innombrables attentions auprès de ton équipe. J'ai beaucoup appris à tes côtés.

A Madame le Dr Morand, de m'avoir fait l'honneur d'accepter de façon si spontanée d'être membre de mon jury de thèse.

Même si nos chemins se sont peu croisés au cours de cet internat, merci pour tes conseils avisés, ton écoute et ton enthousiasme. 


\section{A ma famille,}

\section{A mes parents,}

Merci pour leur soutien sans faille durant ces longues et fastidieuses études dans les bons et moins bons moments. Des textes de sciences-hu des heures entières aux ravitaillements lors des révisions, vous avez toujours su être là pour moi. Vous faites partie intégrante de cette réussite.

A mes sœurs, mon frère, beaux-frères, belle-sœur et les « six nains »,

Merci de m'avoir supportée avec ma bonne humeur, toujours soutenue et appris à avancer coûte que coûte.

J'aurai, je l'espère, plus de temps à partager avec vous !

Je suis fière de faire partie d'une famille si unie.

A ma cousine, qui malgré la distance aurait dû être là en ce jour si important, me touche beaucoup. Je suis fière de voir la belle personne que tu es devenue.

A mon oncle, mes tantes, et marraine merci pour votre soutien depuis toujours. 


\section{A mes ami(e)s,}

Aux éternelles, présentes des cours de récré aux bancs de la faculté.

Musmus, merci pour ton amitié qui brave maintenant les dizaines d'années, pour ton soutien pendant ce long chemin. Je suis toujours aussi heureuse de te retrouver.

Aude (sans oublier Camillou), pour être présente dans les moments qui comptent depuis toujours.

La BDK ou BDT selon l'humeur du groupe! On s'était donné rendez-vous dans dix ans...On y est, après deux mariages, et trois beaux bébés et des milliers d'autres projets ..

Merci de la place que vous m'accordez dans le groupe par votre amitié.

A tous ces moments importants passés avec vous et à venir, merci d'être encore là aujourd'hui.

\section{Aux zouzes du groupe,}

Mar, quelques lignes seraient bien trop courtes pour résumer cette amitié et tu sais à quel point je suis douée pour ce genre de chose. Alors juste merci pour ta présence à mes côtés des TP de P2 à nos nombreuses cohabitations, toujours là pour de bons petits plats et les mots qu'il faut pour remotiver les troupes, merci pour être et rester toi-même tout simplement.

Clairousse, merci d'avoir été à mes côtés depuis si longtemps, pour ta franchise sans condition et tes bons conseils! Tu fais partie de mes amies les plus proches celles qui rentrent et qui ne s'en vont pas.

Noé, merci pour cette colocation quelque peu improbable, pour ton soutien sans faille ces 6 derniers mois, tes conseils avisés, tes relectures sans rechigner et tes petits plats cuisinés. Reste cette même personne aussi dévouée pour les autres!

$\mathrm{Tu}$ vas pouvoir reprendre ta vie d'avant, je vous souhaite tout le bonheur du monde avec ChriChri d'amour et ...mayko. 
Anita, merci d'être l'amie que tu es, celle que l'on peut ne pas voir des mois entiers et être sûr de pouvoir compter sur elle malgré tout et que rien ne change..

Nat (et Basilou), une des dernières recrues du groupe et pas des moindres, merci pour ta gentillesse et joie de vivre de tout instant.

A Alice, Eve, Marie x 2, et Charlotte que je vois moins souvent mais toujours présentes dans les moments importants.

Aux Zouzons du groupe,

A mon Chick Chick, merci pour ton optimisme à toute épreuve ! A toutes ces heures de sous-colle travaillées ensemble, mais surtout à toutes ces soirées enivrées passées et à venir. Merci d'être là à mes côtés et me laisser être ton amie.

A Alex ou bru, le plus beau, merci pour ton humour, ta sensibilité et ta bonne humeur, à ces « ciné dimanche » partagés ces derniers mois !

A tous les autres zouzons du groupe, Jaroume, Antunettu, Chris, Matthieu, Gui, Louis, Greg, PA, et Flo.

A Cédric malgré tout. 


\section{A mes co-internes de pédiatrie,}

Coco et Vic (sans oublier ta princesse Alice) pour être à mes côtés depuis les premières heures de cet internat sans jamais plus se quitter! Même si chacun de nous a pris des chemins différents vous êtes toujours là pour de longues discussions à refaire la vie, comme pour les apéros (pas plus tard que minuit pour mamie Vicou on sait !) Je suis fière de ce que vous êtes devenues !

A Sophie F., pour ton soutien et ta patience sans faille dès le départ, à tous nos fous rires et nos potins partagés, tu fais une partenaire idéale de travail comme de voyages ! Hâte de partager la suite avec toi..

A Sophie B. à la plus belle des futures mariées, merci pour ta bonne humeur, tes attentions, ton écoute dans les bons et moins bons moments.

A Mimo même si tu n'es pas une vraie pédiatre c'est comme ça que je t'ai connu ! Merci de nous avoir reçu sans hésiter et fait rêver lors de cette escapade à Tahiti. Ravie de m'être rapprochée de toi et encore merci pour cette belle rencontre.

A Yoann (j'espère que c'est la bonne écriture!!),

Merci pour tout ces allers-retours sans question, tes multiples attentions, ta simplicité au quotidien et pour toutes les autres choses que j'aime chez toi. Le meilleur reste devant nous.. 


\section{A mes co-internes de dermato,}

Bi, Camille, Shanon, Jess et Anastasia, merci d'avoir été autant mes confidentes que mes enseignantes de dermato, d'avoir supporté mes doutes et mon humeur. Vous m'avez permis et fait sentir comme l'une des vôtres dans cette spécialité. J'ai énormément appris grâce à vous !!

Je vous souhaite pleins pleins de belles choses pour l'avenir. Vous êtes formidables comme dirait une certaine $\mathrm{Bi}$.

A toute l'équipe médicale et paramédicale du service de dermatologie. Un remerciement particulier à Madame le Dr Monestier pour son investissement dans notre enseignement des soirées entières, pour ses connaissances historiques et son amour pour l'anglais.

Je remercie également les assistants du service Max, Marie, Gladys, et le Professeur Amatore pour la confiance qu'ils ont su m'accorder et de m'avoir tant appris ces six derniers mois.

A tous les médecins que j’ai côtoyés pendant mon internat, à l'hôpital, en ville ou à la faculté.

Merci spécialement aux meilleurs assistants de pédiatrie : Dr Marine Juzaud, Dr Stéphanie Clavé et Dr Urbina pour le plaisir d'avoir travaillé à vos côtés, pour m'avoir donné envie de faire cette belle spécialité, pour vos bons conseils et pour m'avoir aidé à devenir le médecin que je suis aujourd'hui.

Un merci particulier à Mme Pauly Vanessa pour son aide précieuse sur les statistiques (on partait de loin !) et sa réactivité.

Une spéciale dédicace au Covid-19 ou Coronavirus, merci d'avoir fait de ce moment qui aurait dû être un partage avec ma famille et mes amis...un huit clos. Je pense me souvenir encore longtemps de toi. 
"La force qui est en chacun de nous est notre plus grand médecin. »

Hippocrate 


\section{Table des matières}

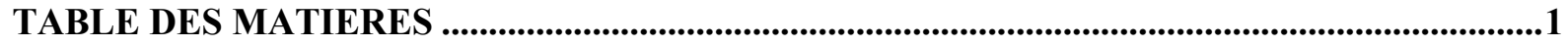

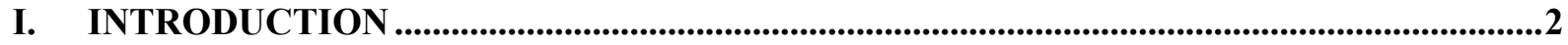

II. MATERIELS ET METHODES..................................................................................................4

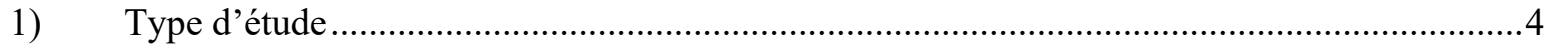

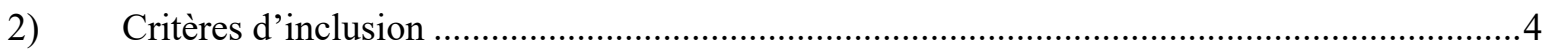

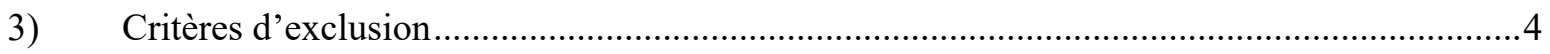

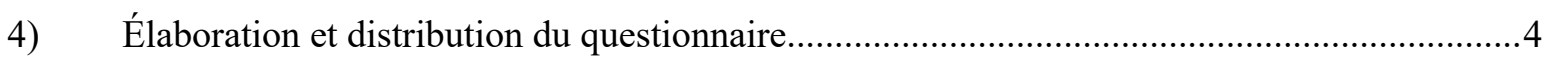

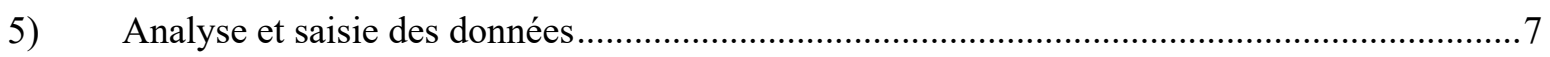

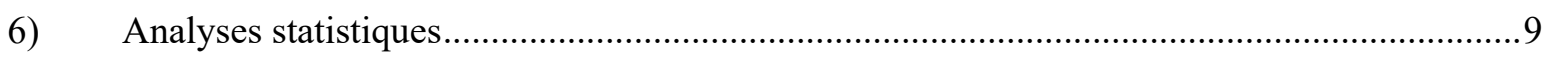

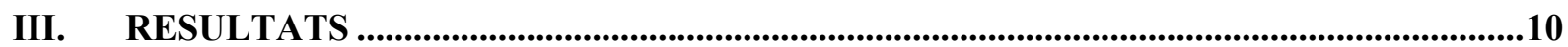

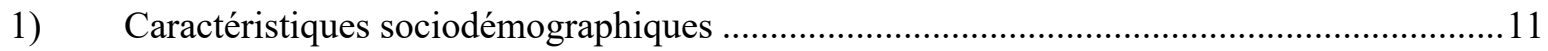

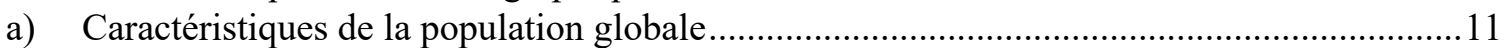

b) Caractéristiques de la population DA et autres pathologies de dermatologie pédiatrique.12

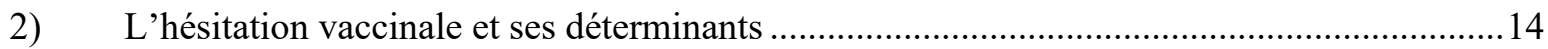

a) Hésitation vaccinale et ses déterminants dans la population globale .....................................14

b) Hésitation vaccinale et ses déterminants dans la population DA .......................................24

3) Lien entre l'HV et la corticophobie dans la population DA..................................................28

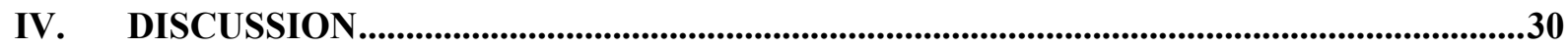

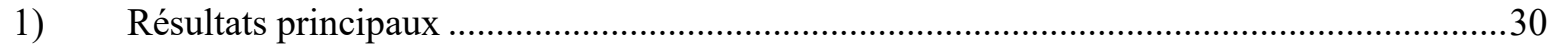

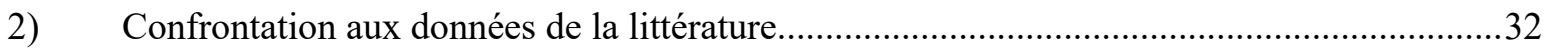

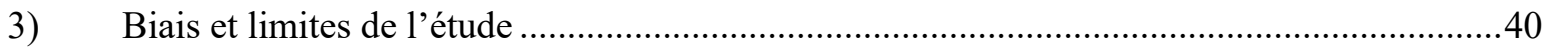

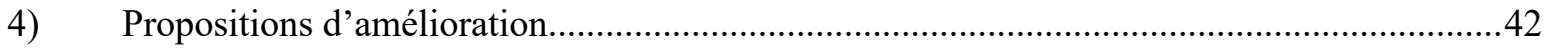

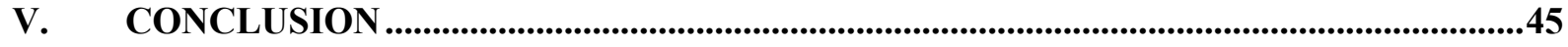

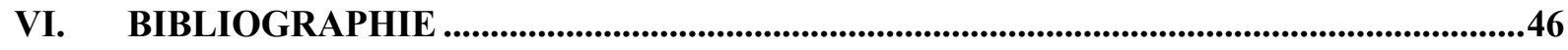

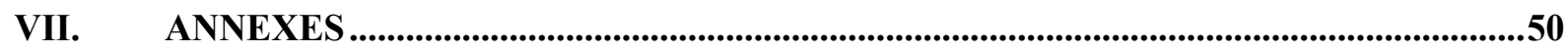

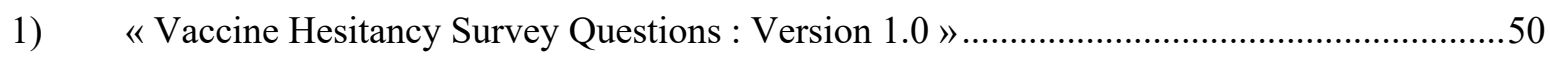

2) Questionnaire : « La vaccination chez les enfants suivis en dermatologie pédiatrique » .........1

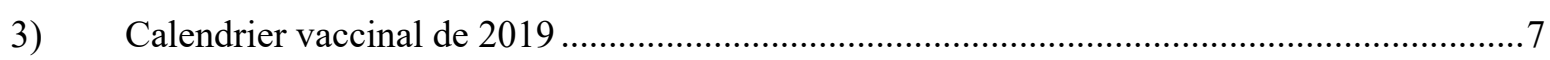

4) Modèle des déterminants de l'hésitation vaccinale mis en place par l'OMS ..........................8

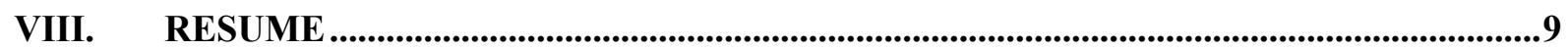




\section{INTRODUCTION}

Au cours de ces dernières années, il s'est développé en France une baisse de la confiance de la population vis-à-vis des vaccins alors qu'ils représentent l'un des aspects les plus importants des soins préventifs pédiatriques (1). L'Organisation Mondiale de la Santé (OMS) estime que les vaccins ont permis d'éviter 2 à 3 millions de décès (2).

La vaccination a toujours fait l'objet de contestations (3), mais aujourd'hui son audience s'est considérablement étendue du fait de l'hypermédiatisation de controverses vaccinales (4).

Aujourd'hui, si plus de trois quarts de la population a une opinion favorable sur la vaccination en général: 75,1 \% en 2016 et 77, 7\% en 2018 d'après les baromètres consécutifs menés par Santé publique France, ces chiffres restent cependant très en deçà des niveaux d'adhésion observés jusqu'au début des années 2000 (5).

En 2012 le groupe stratégique consultatif d'experts sur la vaccination de l'OMS, the Strategic Advisory Group of Experts on immunisation (SAGE) a pris conscience du phénomène et a défini l'hésitation vaccinale (HV) comme le "retard à l'acceptation ou le refus des vaccinations malgré l'existence d'un dispositif d'offre vaccinale »(6)(7). Ainsi, la couverture vaccinale dans une population n'est pas le reflet fidèle de l'HV, mais sa diminution entraine une baisse de l'immunité de groupe et donc l'apparition de nouvelles épidémies. Ce fut le cas pour la rougeole multipliée par quatre au premier trimestre 2019 par rapport à la même période l'an dernier (8), la région Provence Alpes Côte d'Azur (PACA) ayant été une des régions les plus touchées en France (9).

Dans une étude menée en 2016 dans 67 pays, Larson et al. révélaient que l'Europe présentait le taux le plus élevé d'avis négatifs concernant l'importance, la sécurité et l'efficacité des vaccins et que la France était le pays le plus sceptique quant à la sécurité des vaccins avec $41 \%$ d'avis négatifs pour une moyenne mondiale de $13 \%$ (10).

Les réticences à l'égard de la vaccination se retrouvent aussi chez les professionnels de santé, comme les médecins généralistes à l'origine de $90 \%$ des prescriptions vaccinales (11). Entre 16 et $43 \%$ d'entre eux ne recommanderaient jamais ou seulement parfois certains vaccins aux populations cibles (12). Cette hésitation du corps médical peut renforcer celle des parents qui a été évalué par l'enquête du «Baromètre santé » en 2016 à $46 \%$ dans le groupe des parents d'enfants âgés de un à quinze ans (13). 
Parallèlement à cela, la prévalence des maladies atopiques (asthme, eczéma ou dermatite atopique (DA), rhinite et conjonctivite allergique) a considérablement augmenté au cours de ces trente dernières années non seulement dans la plupart des pays occidentaux mais partout dans le monde (14).

La DA est la plus fréquente et la plus précoce des manifestations cliniques associées à l'atopie (15). C'est une dermatose chronique prurigineuse évoluant par poussées, dont l'origine reste multi factorielle, qui a pour conséquences un prurit invalidant retentissant sur le sommeil (16), la qualité de vie de l'enfant et de sa famille (17). C'est un véritable enjeu de santé publique (18).

La fréquence de la DA a doublé voire triplé en 30 ans et toucherait 10 à $25 \%$ des enfants (19)(14). Les raisons de cette tendance ne sont pas entièrement comprises. Cependant, il est évident qu'elle est le résultat de facteurs environnementaux et non d'un changement de disposition génétique (20). La vaccination reste souvent la première incriminée par les parents d'enfants atteints de DA (21)(22).

Ils sont particulièrement préoccupés par la crainte qu'elle puisse favoriser le développement, aggraver la maladie ou encore entrainer des effets secondaires graves comme l'ont suggéré certains rapports (23), ce qui pourrait entrainer une vaccination incomplète ou retardée chez certains enfants.

Le traitement principal de la DA est la corticothérapie locale (24). Les dermocorticoïdes ont prouvé leur efficacité, tolérance, utilité avec un recul depuis les années 1950. Malgré cela ils sont la cible de corticophobie chez les parents qui est la crainte, la peur voire le refus de leur utilisation et est responsable d'une mauvaise observance, source principale d'échec thérapeutique (25)(26). Aucune étude n'a mis en évidence que ces parents étaient plus « vaccinophobes » que la moyenne de la population ni qu'il y avait un lien entre cette crainte des dermocorticoïdes et l'HV. L'amélioration de nos connaissances sur les groupes de population les plus vulnérables à l'HV est une condition préalable à la conception d'interventions adaptées pour y remédier.

Notre étude a pour objectif principal d'évaluer l'HV auprès des parents d'enfants atteints d'une DA par rapport aux autres pathologies de dermatologie pédiatrique.

Les objectifs secondaires sont de connaître les déterminants de cette HV et de rechercher un lien avec la corticophobie dans la population de parents d'enfants atteints de DA. 


\section{MATERIELS ET METHODES}

\section{1) Type d'étude}

Notre étude était une étude observationnelle, prospective, uni centrique réalisée sur une période de trois mois entre le 18 Novembre 2019 et le 14 Février 2020, dans le service de dermatologie du Pr Grob au sein du centre hospitalier universitaire de la Timone à Marseille (APHM) auprès de parents emmenant leurs enfants de moins 18 ans.

\section{2) Critères d'inclusion}

Les critères d'inclusion étaient : être parent ou tuteur légal d'au moins un enfant de moins de 18 ans, suivi ou venant pour la première fois en dermatologie à la Timone dans le service du $\operatorname{Pr}$ Grob, aussi bien en consultation qu'en hospitalisation conventionnelle, hôpital de jour ou atelier d'éducation thérapeutique du patient (ETP).

Avoir donné son consentement oral et être capable de le faire.

Savoir lire et comprendre la langue française.

\section{3) Critères d'exclusion}

Les critères d'exclusion étaient: le refus d'un parent ou tuteur légal de répondre au questionnaire ou ne pas savoir lire et/ou écrire la langue française.

\section{4) Élaboration et distribution du questionnaire}

Nous avions choisi d'utiliser un questionnaire en format papier. Un seul questionnaire était fourni par famille, et rempli par un des deux parents. Si les parents venaient consulter pour plusieurs enfants, il était précisé de répondre uniquement pour le plus jeune d'entre eux.

Tous les participants étaient informés des objectifs de l'étude et de la confidentialité des données. Le consentement était considéré comme implicite chez les parents qui avaient rempli volontairement et anonymement le questionnaire. Un pré-test avait été réalisé sur une dizaine de personnes extérieures au service choisies au hasard. Ce qui nous a permis de corriger et valider notre outil de collecte des données notamment sur le niveau de compréhension de chaque item, l'acceptabilité et le temps nécessaire pour y répondre. 
Il comprenait 37 questions, incluant trois thématiques :

1) Les données épidémiologiques sur les parents avec leur âge, sexe, niveau d'étude et catégorie socio-professionnelle selon la nomenclature des professions et catégories socioprofessionnelles de l'INSEE (PCS-2017) (27).

2) Les données épidémiologiques concernant l'enfant et sa pathologie dermatologique avec l'âge, le sexe, la maladie de peau pour laquelle les parents venaient consulter (DA ou autres) et s'ils avaient eu recours à des médecines alternatives qui est selon l'OMS : « un vaste ensemble de pratiques de soins de santé qui n'appartient pas à la tradition du pays et ne sont pas intégrées dans le système de santé dominant».

Si leur enfant avait une DA, on leur demandait s'ils avaient une autre maladie atopique associée, s'ils avaient participé à un atelier d'ETP qui est selon la définition de l'OMS : « un processus intégré aux soins qui a pour objectif d'améliorer la prise en charge des patients en les aidant à s'autonomiser, à acquérir et à conserver des compétences afin de les aider à vivre de manière optimale leur maladie ».

On évaluait également la corticophobie des parents pour connaitre leur degré d'adhérence visà-vis des corticoïdes locaux grâce à un score multidimensionnel appelé TOPIcal COrticosteroid Phobia (TOPICOP) qui comprenait 12 items dont les 6 premiers évaluaient les croyances des parents vis-à-vis des dermocorticoïdes et les 6 derniers leurs peurs. Ce score a été validé sur le plan national en 2013 (28) et sur le plan international en 2017 (29).

3) Les données concernant la vaccination de leur enfant avec un questionnaire d'HV appelé «Vaccine Hesitancy Survey question » (VHS), crée par le groupe de travail SAGE de l'OMS et validé en 2014 après essais pilotes au niveau mondial et revue de la littérature (6)(30). Les questions ont été adaptées à partir de l'enquête sur les attitudes des parents à l'égard des vaccins de l'enfance («Parent Attitudes about Childhood Vaccines survey » ou PACV), précédemment développée par Opel et al. (31) et se sont avérées valables et fiables dans une population à haut revenu. Nous avons utilisé uniquement le questionnaire à choix fermé comprenant 10 items avec des réponses majoritairement dichotomiques «oui/non » sauf pour 4 questions où si les parents répondaient « oui », ils pouvaient s'exprimer en donnant un exemple. 
Ce questionnaire est présenté en annexe 1.

Il a été traduit de l'anglais au français par un interprète natif du pays et spécialisé dans le langage médical. Quelques modifications ont été apportées afin de préciser le statut vaccinal de l'enfant à la question 26.

Nous avions mis sur la même page le schéma du dernier calendrier vaccinal français en vigueur de 2019 selon Santé publique France, présenté en annexe 3, en faisant un rappel sur les vaccinations obligatoires depuis le $1^{\text {er }}$ janvier 2018 et celles recommandées (32). Les parents pouvaient s'ils le souhaitaient, découper ce schéma et le garder pour mieux comprendre et suivre la vaccination de leurs enfants.

Les autres modifications portaient sur les vaccins énumérés à la question 31 afin de correspondre aux noms commerciaux des vaccins disponibles sur le marché pour faciliter la compréhension des parents.

Les questionnaires étaient distribués par l'agent administratif au moment de l'enregistrement du parent pour la consultation ou hospitalisation de leur enfant. Puis ils étaient recueillis par les agents d'accueil avant la consultation pour ne pas influencer les réponses.

Le questionnaire était auto-déclaratif : aucune vérification des affirmations des patients n'était faite, aucun carnet de vaccination n'était demandé, seulement recommandé à la question 26 concernant le statut vaccinal de l'enfant.

Le questionnaire ainsi modifié est présenté en annexe 2.

Les données des questionnaires ont été collectées sur fichier Excel au fur et à mesure de l'étude. 


\section{5) Analyse et saisie des données}

Les parents dit «hésitants vaccinaux » selon la définition de l'OMS correspondaient aux individus ayant déjà retardé et/ou refusé un vaccin, et donc aux individus ayant répondu « oui » à la question 29 ("Avez-vous hésité à faire vacciner votre enfant? 》) et/ou 30 ("Avez-vous refusé à faire vacciner votre enfant?»).

Les parents non hésitants correspondaient aux individus ayant répondu « non » à la question 29 et à la question 30 .

Pour éviter toute confusion avec les parents dits " hésitants vaccinaux 》 comme d'après la définition de l'HV de l'OMS, le terme « hésité » a été remplacé par « retard à l'acceptation d'un vaccin ».

Le statut vaccinal de l'enfant était considéré « à jour » lorsqu'il était vacciné selon tous les vaccins obligatoires et recommandés du calendrier vaccinal pour son âge (annexe 3). C'est-àdire lorsque les parents ont répondu «Tout ses vaccins obligatoires ou non sont à jour » à la question 26.

Nous avons considéré que l'enfant n'était « pas à jour » de ses vaccins, si les parents avaient répondu : « ses vaccins sont partiellement à jour (il manque au moins une dose pour les vaccins obligatoires) 》 ou « uniquement les vaccins obligatoires ou « il n'est pas vacciné».

Les réponses libres aux questions 34, 35, 36 ont été classées selon le modèle des déterminants de l'HV de l'OMS proposait à l'issue de son rapport en 2014 sur la base d'un examen systématique de la littérature et adapté à la population française (7)(33).

Regroupant trois grands groupes :

- Les influences contextuelles

- Les influences individuelles et de groupe

- Les déterminants liés de façon spécifiques à un vaccin donné ou à la vaccination

Le modèle est décrit en détail en annexe 4.

La corticophobie des parents était évaluée grâce au score TOPICOP. Quatre choix de réponses étaient proposés, de totalement en désaccord à totalement d'accord, avec des points attribués à chacun $(0,1,2$ ou 3$)$, les valeurs plus élevées correspondaient à une corticophobie plus sévère. Le score maximal était de 36 , exprimé en pourcentage. 
Nous avons considéré également que le fait de répondre « oui » à la question numéro 13 :« Avez-vous des craintes ou réticences vis-à-vis des crèmes à base de cortisone ? " était prédictif de corticophobie chez ces parents-là. 


\section{6) Analyses statistiques}

Dans un premier temps, nous avons réalisé une analyse descriptive de l'ensemble de la population puis de la population de parents d'enfants souffrant de DA.

Les données quantitatives ont été décrites via la moyenne et l'écart type : les données qualitatives via les effectifs et pourcentages.

Pour étudier les facteurs liés à l'hésitation vaccinale dans la population générale puis plus spécifiquement dans la population DA, nous avons utilisé 2 modèles de régression logistique binaire multiple. Nous avons utilisé une stratégie de modélisation descendante : les variables significatives au seuil alpha $<0.20$ en régression logistique uni-factorielle ont d'abord été introduites dans le modèle multiple et une procédure d'élimination pas à pas des variables a été réalisée jusqu'à l'obtention de variables significativement liées à l'hésitation vaccinale au seuil de 0.05. Les résultats sont présentés sous formes d'odds ratio (OR) avec leur intervalle de confiance à 95\% (IC 95\%).

Le seuil de significativité a été fixe à alpha $=0.05$ et les statistiques ont été réalisés à l'aide du logiciel SPSS v20.

Nous avons obtenu l'avis favorable du comité d'éthique du centre hospitalier de la Timone de Marseille.

Les participants n'ont reçu aucune forme de rémunération pour leur participation à cette étude. 


\section{RESULTATS}

Entre le 18 novembre 2019 et le 14 février 2020, 330 questionnaires ont été distribués et 322 collectés dans le service de dermatologie de la Timone à Marseille (consultation, hôpital de jour et hospitalisation conventionnelle). Nous avons recensé un total de 452 consultations de dermatologie pédiatrique, 2 hospitalisations, 12 hôpitaux de jour, et 41 ETP durant ces dates. Soit un total de 507 séjours en service de dermatologie chez les enfants de moins de 18 ans.

Huit parents ont refusé de répondre au questionnaire parce qu'ils ne savaient pas lire et/ou écrire la langue Française et dix questionnaires ont été exclus car ils étaient incomplets concernant les données du critère de jugement principal. Le taux global de participation était estimé à 94,5\% . Notre échantillon était réparti selon le flow chart suivant (figure 1).

Figure 1: Flow chart

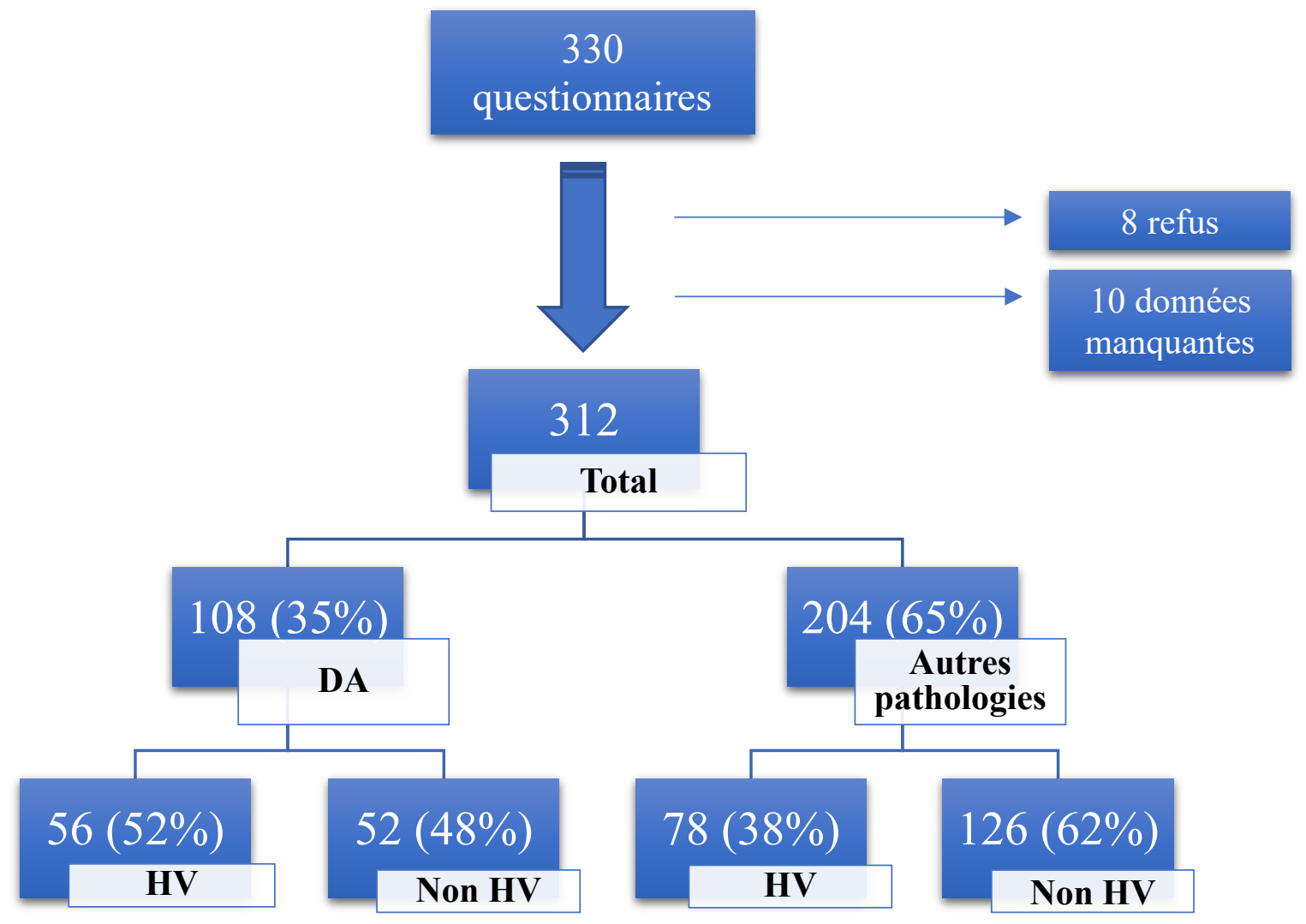

$D A$ : dermatite atopique, $H V$ : hésitants vaccinaux 


\section{1) Caractéristiques sociodémographiques}

\section{a) Caractéristiques de la population globale}

Les caractéristiques sociodémographiques de l'échantillon sont résumées dans le tableau 1.

Tableau 1 : Caractéristiques sociodémographiques de l'échantillon

\begin{tabular}{|c|c|c|c|}
\hline & & $\begin{array}{c}\text { Effectif } \\
\mathrm{N}=312\end{array}$ & Fréquence $(\%)$ \\
\hline \multicolumn{4}{|c|}{ Caractéristiques du parent } \\
\hline \multirow{3}{*}{ Age } & $<20$ ans & 5 & 2 \\
\hline & $20-40$ ans & 195 & 63 \\
\hline & 40-60 ans et plus & 112 & 36 \\
\hline \multirow{2}{*}{ Sexe } & Homme & 68 & 22 \\
\hline & Femme & 244 & 78 \\
\hline \multirow{4}{*}{ Profession } & Employé / ouvrier & 128 & 41 \\
\hline & Cadre / profession intermédiaire & 90 & 29 \\
\hline & Artisans / commerçants / agriculteurs / chef d'entreprise & 30 & 10 \\
\hline & Retraité / sans activité / autre & 64 & 21 \\
\hline \multirow{4}{*}{ Niveau d'étude } & $C A P$-BEP-autres & 70 & 22 \\
\hline & Baccalauréat & 55 & 18 \\
\hline & DEUG-BTS-licence & 99 & 32 \\
\hline & Maitrise ou plus & 88 & 28 \\
\hline \multirow{2}{*}{ Médecine alternative } & Oui & 65 & 21 \\
\hline & Non & 247 & 79 \\
\hline \multirow{2}{*}{ HV } & Oui & 134 & 43 \\
\hline & Non & 178 & 57 \\
\hline \multicolumn{4}{|c|}{ Caractéristiques de l'enfant } \\
\hline \multirow{4}{*}{ Age } & $<2$ ans (avant 01/01/18) & 96 & 31 \\
\hline & 2ans - 6 ans (après 01/01/18) & 72 & 23 \\
\hline & 6 ans -12 ans & 92 & 29 \\
\hline & 12 ans - 18 ans & 52 & 17 \\
\hline \multirow{2}{*}{ Sexe } & Garçon & 142 & 46 \\
\hline & Fille & 170 & 54 \\
\hline \multirow{2}{*}{ Motif de consultation } & $D A$ & 108 & 35 \\
\hline & Autres pathologies dermatologiques & 204 & 65 \\
\hline \multirow{4}{*}{ Statut vaccinal } & À jour & 222 & 71 \\
\hline & Uniquement les obligatoires & 45 & 14 \\
\hline & Pas vacciné / partiellement / ne sais pas & 38 & 12 \\
\hline & $N R$ & 7 & 2 \\
\hline
\end{tabular}

$D A$ : dermatite atopique, $H V$ : hésitants vaccinaux, CAP : certificat d'aptitude professionnelle, BEP : brevet d'étude professionnelle, DEUG : diplôme d'études universitaires générales, BTS : brevet de technicien supérieur, NR : non répondu 
Les répondants étaient essentiellement des mères (78\%). La classe d'âge la plus représentée était celle des 20 à 40 ans (63\%). La catégorie socio-professionnelle la plus représentée était celle des employés et/ou ouvriers (41\%). La plupart avait fait des études supérieures. Une minorité pratiquait des médecines alternatives (21\%).

Les parents étaient classés en fonction de ce qu'ils avaient répondu aux questions 29 et 30, et étaient considérés comme « hésitants vaccinaux » ou «non hésitants ». L'hésitation à l'égard des vaccins était partagée dans l'échantillon puisque la moitié (57\%) des parents déclaraient n’avoir jamais refusé et/ou hésité à faire vacciner leur enfant.

Les enfants qui consultaient étaient âgés de moins de 12 ans pour la plupart (83\%), et le sexratio était équilibré $(0,83)$.

Ils consultaient pour une DA dans un $1 / 3$ des cas.

Les parents déclaraient à $71 \%$ que le statut vaccinal de leur enfant était «à jour » (vaccins recommandés et obligatoires).

\section{b) Caractéristiques de la population DA et autres pathologies de dermatologie pédiatrique}

Les caractéristiques sociodémographiques de la population DA et autres pathologies sont représentées par le tableau 2.

Parmi les parents d'enfants atteints de DA, il n'y avait pas de différence statistiquement significative concernant leur âge, sexe, profession et niveau d'étude par rapport à ceux atteints d'autres pathologies dermatologiques.

En revanche, les garçons étaient plus représentés dans la population DA (56\% versus $41 \%$, $\mathrm{p}=0,0012)$, de même que la classe d'âge $6-12$ ans $(37 \%$ versus $25 \%, p=0,002)$. Ceux souffrant d'autres pathologies de dermatologie pédiatrique étaient plus jeunes, puisque plus d'un tiers était âgé de moins de 2 ans (38\% versus $18 \%, \mathrm{p}=0,002)$.

La pratique de médecine alternative était plus fréquente dans la population DA (38\% versus $12 \%, \mathrm{p}<0,001)$.

On ne retrouvait pas de différence significative concernant le statut vaccinal de l'enfant entre les deux populations. 


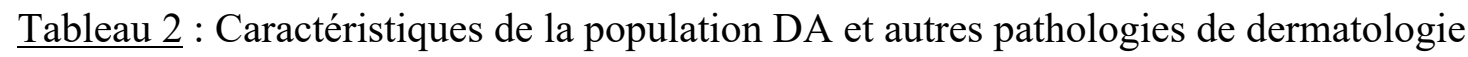

\begin{tabular}{|c|c|c|c|c|c|c|}
\hline & & \multicolumn{2}{|c|}{$\begin{array}{l}\text { Échantillon global } \\
\qquad \mathbf{N}=\mathbf{3 1 2}\end{array}$} & \multirow{2}{*}{$\begin{array}{c}\text { DA } \\
\mathbf{N}=108 \\
\text { n (\%) }\end{array}$} & \multirow{2}{*}{$\begin{array}{c}\begin{array}{c}\text { Autres } \\
\text { Pathologies } \\
\text { N=204 }\end{array} \\
\mathrm{n}(\%)\end{array}$} & \multirow{2}{*}{ p-value } \\
\hline & & $\mathrm{n}$ & $\%$ & & & \\
\hline \multicolumn{7}{|c|}{ Caractéristiques du parent } \\
\hline \multirow{3}{*}{ Age } & $<20$ ans & 5 & 2 & $2(2)$ & $3(1,5)$ & \multirow{3}{*}{0,199} \\
\hline & $20-40$ ans & 195 & 63 & $61(57)$ & $134(66)$ & \\
\hline & 40-60 ans et plus & 112 & 36 & $45(42)$ & $67(33)$ & \\
\hline \multirow{2}{*}{ Sexe } & Homme & 68 & 22 & $26(24)$ & $42(21)$ & \multirow{2}{*}{0,478} \\
\hline & Femme & 244 & 78 & $82(76)$ & $162(79)$ & \\
\hline \multirow{4}{*}{ Profession } & Employé / ouvrier & 128 & 41 & $47(44)$ & $81(40)$ & \multirow{4}{*}{0,559} \\
\hline & Cadre / profession intermédiaire & 90 & 29 & $28(26)$ & $62(30)$ & \\
\hline & $\begin{array}{l}\text { Artisans / commerçants / } \\
\text { agriculteurs / chef d'entreprise }\end{array}$ & 30 & 10 & $13(12)$ & $17(8)$ & \\
\hline & Retraité / sans activité / autre & 64 & 21 & $20(19)$ & $44(22)$ & \\
\hline \multirow{4}{*}{ Niveau d'étude } & $C A P$ - BEP-autre & 70 & 22 & $26(24)$ & $44(22)$ & \multirow{4}{*}{0,803} \\
\hline & Baccalauréat & 55 & 18 & $21(19)$ & $34(17)$ & \\
\hline & DEUG-BTS-licence & 99 & 32 & $31(29)$ & $68(33)$ & \\
\hline & Maitrise ou plus & 88 & 28 & $30(28)$ & $58(28)$ & \\
\hline \multirow{2}{*}{$\begin{array}{l}\text { Médecine } \\
\text { alternative }\end{array}$} & Oui & 65 & 21 & $41(38)$ & $24(12)$ & \multirow{2}{*}{$<0,001^{*}$} \\
\hline & Non & 247 & 79 & $67(62)$ & $180(88)$ & \\
\hline \multicolumn{7}{|c|}{ Caractéristiques de l'enfant } \\
\hline \multirow{4}{*}{ Age } & $<2$ ans (avant 01/01/18) & 96 & 31 & $19(18)$ & $77(38)$ & \multirow{4}{*}{$0,002 *$} \\
\hline & 2ans - 6 ans (après 01/01/18) & 72 & 23 & $29(27)$ & $43(21)$ & \\
\hline & 6 ans -12 ans & 92 & 29 & $40(37)$ & $50(25)$ & \\
\hline & 12 ans -18 ans & 52 & 17 & $20(19)$ & $32(16)$ & \\
\hline \multirow{2}{*}{ Sexe } & Garçon & 142 & 46 & $60(56)$ & $82(41)$ & \multirow{2}{*}{$0,012 *$} \\
\hline & Fille & 170 & 54 & $48(44)$ & $120(59)$ & \\
\hline \multicolumn{7}{|c|}{ Attitudes des parents vis-à-vis des vaccins } \\
\hline \multirow{4}{*}{$\begin{array}{l}\text { Statut vaccinal de } \\
\text { l'enfant }\end{array}$} & $\grave{A}$ jour & 222 & 71 & $75(70)$ & $147(72)$ & \multirow{4}{*}{0,553} \\
\hline & Uniquement les obligatoires & 45 & 14 & $19(18)$ & $26(13)$ & \\
\hline & $\begin{array}{l}\text { Pas vacciné / partiellement / ne } \\
\text { sais pas }\end{array}$ & 38 & 12 & $13(12)$ & $25(13)$ & \\
\hline & $N R$ & 7 & 2 & $1(1)$ & $6(2)$ & \\
\hline \multirow{4}{*}{ HV } & Oui & 134 & 43 & $56(52)$ & $78(38)$ & $0,021^{*}$ \\
\hline & Ont retardé l'acceptation & 125 & 41 & $52(48)$ & $73(37)$ & 0,055 \\
\hline & Ont refusé & 69 & 23 & $33(31)$ & $36(18)$ & $0,014 *$ \\
\hline & Non & 178 & 57 & $52(48)$ & $126(62)$ & $0,021 *$ \\
\hline
\end{tabular}

$D A$ : dermatite atopique, $H V$ : hésitants vaccinaux, $C A P$ : certificat d'aptitude professionnelle, BEP : brevet d'étude professionnelle, DEUG : diplôme d'études universitaires générales, BTS : brevet de technicien supérieur, NR : non répondu 


\section{2) L'hésitation vaccinale et ses déterminants}

Au total $43 \%$ des parents interrogés ont déclaré être « hésitants vaccinaux » c'est-à-dire ayant déjà refusé et/ou retardé à accepter un vaccin pour leur enfant (tableau 2).

Cinquante-deux pour cent des parents d'enfants atteints de DA étaient hésitants, contre $38 \%$ des parents d'enfants atteints d'une autre pathologie dermatologique. Cette différence était statistiquement significative $(\mathrm{p}=0,0021)$. Les parents d'enfants souffrants de DA exprimaient plus de refus catégorique ( $31 \%$ versus $18 \%, \mathrm{p}=0,0014)$.

Les déterminants de l'HV sont représentés par les tableaux 3 et 4 pour les deux populations.

\section{a) Hésitation vaccinale et ses déterminants dans la population globale}

L'HV n'était pas associée de façon significative au niveau d'étude des parents ni sur leurs autres caractéristiques sociodémographiques. En revanche, elle était associée à leurs professions $(\mathrm{p}=<0,001)$ (tableau 3).

On notait plus d'HV s'ils pratiquaient une médecine alternative telle que l'homéopathie, naturopathie, phytothérapie, acupuncture, réflexologie avec une association statistiquement significative $(28 \%$ versus $15 \%, \mathrm{p}=0,006)$.

Parmi les enfants de parents dits « hésitants vaccinaux », seulement $61 \%$ étaient à jour de leurs vaccinations (obligatoires et recommandés) contre 79\% chez les parents dits «non hésitants » $(\mathrm{p}<0,001)$, avec une plus grande proportion d'enfants vaccinés uniquement par les vaccins obligatoires (25\% versus $7 \%)$.

Les autres déterminants de l'HV ont été analysés à partir des questions 27, 28 et à partir de la 31 (tableau 4).

A la question 27 «Pensez-vous que les vaccins peuvent protéger les enfants de maladies graves ? », près de $90 \%$ des parents au total ont répondu « oui », on note cependant un plus fort taux de réponse négative chez les hésitants $(19 \%$ versus $6 \%, \mathrm{p}=0,001)$.

Concernant la question 28 «Pensez-vous que la majorité des parents vaccine ses enfants selon le calendrier vaccinal, c'est-à-dire avec tous les vaccins recommandés? ", sur les 134 parents hésitants 61\% avaient répondu « oui » comme la plupart de l'échantillon (65\%). Le fait de percevoir l'immunisation comme norme sociale n'influençait pas l'hésitation à faire vacciner son enfant dans cette étude. 
$\underline{\text { Tableau } 3}$ : Déterminants de l'hésitation vaccinale dans la population globale et DA

\begin{tabular}{|c|c|c|c|c|c|c|c|c|}
\hline & & $\begin{array}{c}\text { Échantillon } \\
\text { global } \\
\mathbf{N}=312\end{array}$ & $\begin{array}{c}\text { HV } \\
\mathrm{N}=134\end{array}$ & $\begin{array}{c}\text { Non } \\
\text { HV } \\
\mathrm{N}=178\end{array}$ & \multirow[t]{2}{*}{ p-value } & $\begin{array}{c}\text { HV chez } \\
\text { DA } \\
\mathbf{N}=\mathbf{5 6}\end{array}$ & $\begin{array}{c}\text { Non HV } \\
\text { DA } \\
\mathbf{N}=\mathbf{5 2}\end{array}$ & \multirow[t]{2}{*}{ p-value } \\
\hline & & n(\%) & $\mathbf{n}(\%)$ & n(\%) & & $\mathbf{n}(\%)$ & n(\%) & \\
\hline \multicolumn{9}{|c|}{ Caractéristiques du parent } \\
\hline \multirow{3}{*}{ Age } & $<20$ ans & $5(2)$ & $0(0)$ & $5(3)$ & \multirow{3}{*}{0,078} & $0(0)$ & $2(4)$ & \multirow{3}{*}{0,298} \\
\hline & $20-40$ ans & $195(63)$ & $81(60)$ & $114(64)$ & & $30(54)$ & $31(60)$ & \\
\hline & 40-60 ans et plus & $112(36)$ & $53(40)$ & $59(33)$ & & $26(47)$ & $19(37)$ & \\
\hline \multirow{2}{*}{ Sexe } & Homme & $68(22)$ & $27(20)$ & $41(23)$ & \multirow{2}{*}{0,541} & $14(25)$ & $12(23)$ & \multirow{2}{*}{0,815} \\
\hline & Femme & $244(78)$ & 107(80) & 137(77) & & $42(75)$ & $40(77)$ & \\
\hline \multirow{4}{*}{ Profession } & Employé / ouvrier & $128(41)$ & $61(46)$ & $67(38)$ & \multirow{4}{*}{$<0,001^{*}$} & $29(52)$ & $18(35)$ & \multirow{4}{*}{$0,003 *$} \\
\hline & $\begin{array}{l}\text { Cadre / profession } \\
\text { intermédiaire }\end{array}$ & $90(29)$ & $28(21)$ & $62(35)$ & & $10(18)$ & $18(35)$ & \\
\hline & $\begin{array}{l}\text { Artisans / } \\
\text { commerçants / } \\
\text { agriculteurs / chef } \\
\text { d'entreprise }\end{array}$ & $30(10)$ & 21(16) & $9(5)$ & & $11(20)$ & $2(4)$ & \\
\hline & $\begin{array}{l}\text { Retraité / sans } \\
\text { activité / autre }\end{array}$ & $64(21)$ & $24(18)$ & $40(22)$ & & $6(11)$ & $14(27)$ & \\
\hline \multirow{4}{*}{$\begin{array}{l}\text { Niveau } \\
\text { d'étude }\end{array}$} & $C A P$ - BEP-autre & $70(22)$ & $27(20)$ & $43(24)$ & \multirow{4}{*}{0,609} & $12(21)$ & $13(25)$ & \multirow{4}{*}{0,654} \\
\hline & Baccalauréat & $55(18)$ & $26(19)$ & $29(16)$ & & $13(23)$ & $8(15)$ & \\
\hline & DEUG-BTS-licence & $99(32)$ & $46(34)$ & $53(30)$ & & $17(30)$ & $14(27)$ & \\
\hline & Maitrise ou plus & $88(28)$ & $35(26)$ & $53(30)$ & & $14(25)$ & $17(15)$ & \\
\hline \multirow{2}{*}{$\begin{array}{l}\text { Médecine } \\
\text { alternative }\end{array}$} & Oui & $65(21)$ & $38(28)$ & $27(15)$ & \multirow{2}{*}{$0,006^{*}$} & $25(45)$ & $16(31)$ & \multirow{2}{*}{0,138} \\
\hline & Non & $247(79)$ & $97(72)$ & $150(84)$ & & $31(55)$ & $36(69)$ & \\
\hline \multicolumn{9}{|c|}{ Caractéristiques de l'enfant } \\
\hline \multirow{4}{*}{ Age } & $\begin{array}{l}<2 \text { ans (avant } \\
01 / 01 / 18 \text { ) }\end{array}$ & $96(31)$ & $38(28)$ & $58(33)$ & \multirow{4}{*}{0,752} & $9(16)$ & $10(19)$ & \multirow{4}{*}{0,158} \\
\hline & $\begin{array}{l}2 a n s-6 \text { ans (après } \\
01 / 01 / 18)\end{array}$ & $72(23)$ & $30(22)$ & $42(24)$ & & $11(20)$ & $18(35)$ & \\
\hline & 6 ans -12 ans & $90(29)$ & $42(31)$ & $48(27)$ & & $22(39)$ & $18(35)$ & \\
\hline & 12 ans -18 ans & $52(17)$ & $24(18)$ & $28(16)$ & & $14(25)$ & $18(35)$ & \\
\hline \multirow{2}{*}{ Sexe } & Garçon & $142(46)$ & $56(42)$ & $86(48)$ & \multirow{2}{*}{0,257} & $28(50)$ & $32(62)$ & \multirow{2}{*}{0,228} \\
\hline & Fille & $168(54)$ & $77(57)$ & $91(51)$ & & $28(50)$ & $20(38)$ & \\
\hline \multirow{4}{*}{$\begin{array}{l}\text { Statut } \\
\text { vaccinal de } \\
\text { l'enfant }\end{array}$} & $\grave{A}$ jour & $222(71)$ & $82(61)$ & $140(79)$ & \multirow{4}{*}{$<0,001^{*}$} & $32(57)$ & $43(83)$ & \multirow{4}{*}{$0,009 *$} \\
\hline & $\begin{array}{l}\text { Uniquement les } \\
\text { obligatoires }\end{array}$ & $45(14)$ & $33(25)$ & $12(7)$ & & $14(25)$ & $5(10)$ & \\
\hline & $\begin{array}{l}\text { Pas vacciné / } \\
\text { partiellement / ne } \\
\text { sais pas }\end{array}$ & $38(12)$ & $19(14)$ & $19(11)$ & & $11(20)$ & $3(6)$ & \\
\hline & $N R$ & $7(2)$ & $0(0)$ & $7(4)$ & & $0(0)$ & $1(2)$ & \\
\hline
\end{tabular}

DA : dermatite atopique, $H V$ : hésitants vaccinaux, CAP : certificat d'aptitude professionnelle, BEP : brevet d'étude professionnelle, DEUG : diplôme d'études universitaires générales, BTS : brevet de technicien supérieur, ETP : éducation thérapeutique du patient, $N R$ : non répondu 
$\underline{\text { Tableau } 4}$ : Suite des déterminants de l'HV dans la population globale et DA

\begin{tabular}{|c|c|c|c|c|c|c|c|c|}
\hline & & $\begin{array}{c}\text { Échantillon } \\
\text { global } \\
\mathbf{N}=312\end{array}$ & $\begin{array}{c}\text { HV } \\
\mathrm{N}=134\end{array}$ & $\begin{array}{c}\text { Non } \\
\text { HV } \\
\text { N=178 }\end{array}$ & \multirow[t]{2}{*}{ p-value } & $\begin{array}{c}\text { HV } \\
\text { chez } \\
\text { DA } \\
\text { N=56 }\end{array}$ & $\begin{array}{c}\text { Non HV } \\
\text { DA } \\
\mathbf{N}=\mathbf{5 2}\end{array}$ & \multirow[t]{2}{*}{$\begin{array}{c}p- \\
\text { value }\end{array}$} \\
\hline & & $n(\%)$ & $n(\%)$ & $n(\%)$ & & $n(\%)$ & $n(\%)$ & \\
\hline \multirow{3}{*}{$\begin{array}{l}\text { Pense que les vaccins protègent } \\
\text { des maladies graves }\end{array}$} & Oui & $270(87)$ & $109(82)$ & $161(91)$ & \multirow{3}{*}{$0,001 *$} & $48(86)$ & $51(98)$ & \multirow{3}{*}{$0,033 *$} \\
\hline & Non & $36(12)$ & $25(19)$ & $11(6)$ & & $8(14)$ & $1(2)$ & \\
\hline & $N R$ & $6(2)$ & $0(0)$ & $6(3)$ & & $0(0)$ & $0(0)$ & \\
\hline \multirow{4}{*}{$\begin{array}{l}\text { Pense que la majorité des } \\
\text { parents vaccinent leurs enfants } \\
\text { selon les recommandations }\end{array}$} & Oui & & & & \multirow{4}{*}{0,116} & & & \multirow{4}{*}{0,918} \\
\hline & & $202(05)$ & $02(01)$ & $120(0 /)$ & & $5 J(02)$ & $55(05)$ & \\
\hline & Non & $104(34)$ & $52(39)$ & $52(29)$ & & $21(38)$ & $19(37)$ & \\
\hline & $N R$ & $6(2)$ & $0(0)$ & $6(3)$ & & $0(0)$ & $0(0)$ & \\
\hline \multirow{3}{*}{$\begin{array}{l}\text { La distance, les horaires } \\
\text { d'ouverture, le temps d'attente, } \\
\text { ou le coût du transport vous } \\
\text { ont-ils empêché de faire } \\
\text { vacciner votre enfant? }\end{array}$} & Oui & $4(1)$ & $1(1)$ & $3(2)$ & \multirow{3}{*}{0,442} & $\begin{array}{c}56 \\
(100)\end{array}$ & $52(100)$ & \multirow{3}{*}{ - } \\
\hline & Non & $301(96)$ & 133(99) & $168(94)$ & & $0(0)$ & $0(0)$ & \\
\hline & $N R$ & $7(2)$ & $0(0)$ & $7(4)$ & & $0(0)$ & $0(0)$ & \\
\hline \multirow{3}{*}{$\begin{array}{l}\text { Autres difficultés dans votre } \\
\text { vie qui retardent ou ont retardé } \\
\text { la vaccination? }\end{array}$} & Oui & $27(9)$ & $19(14)$ & $8(4)$ & \multirow{3}{*}{$0,004 *$} & $9(16)$ & $6(12)$ & \multirow{3}{*}{0,496} \\
\hline & Non & 278(89) & $115(86)$ & 163(93) & & 47 (84) & $46(88)$ & \\
\hline & $N R$ & $7(2)$ & $0(0)$ & $7(4)$ & & $0(0)$ & $0(0)$ & \\
\hline \multirow{3}{*}{$\begin{array}{l}\text { Autres raisons pour lesquelles } \\
\text { les enfants ne devraient pas } \\
\text { être vaccinés? }\end{array}$} & Oui & $31(10)$ & $27(20)$ & $4(2)$ & \multirow{3}{*}{$<0,001^{*}$} & $12(21)$ & $1(2)$ & \multirow{3}{*}{$0,002 *$} \\
\hline & Non & $274(88)$ & $107(80)$ & $167(94)$ & & 44 (79) & $51(98)$ & \\
\hline & $N R$ & $7(2)$ & $0(0)$ & $7(4)$ & & $0(0)$ & $0(0)$ & \\
\hline \multirow{3}{*}{$\begin{array}{l}\text { Avez-vous déjà reçu ou } \\
\text { entendu des informations } \\
\text { négatives sur les vaccins? }\end{array}$} & Oui & $201(64)$ & 106(79) & $95(53)$ & \multirow{3}{*}{$<0,001^{*}$} & $46(82)$ & $32(63)$ & \multirow{3}{*}{$0,043 *$} \\
\hline & Non & $103(33)$ & $28(21)$ & $75(42)$ & & $10(20)$ & $20(37)$ & \\
\hline & $N R$ & $8(3)$ & $0(0)$ & $8(4)$ & & $0(0)$ & $0(0)$ & \\
\hline \multirow{2}{*}{$\begin{array}{l}\text { Les avez-vous quand même } \\
\text { vaccinés après avoir entendu } \\
\text { ces informations? }\end{array}$} & Oui & 173(86) & $83(78)$ & $90(95)$ & \multirow{2}{*}{$<0,001^{*}$} & 31(69) & 31 (96) & \multirow{2}{*}{$0,002 *$} \\
\hline & Non & $28(14)$ & $23(22)$ & $5(5)$ & & $14(31)$ & $2(4)$ & \\
\hline \multirow{16}{*}{$\begin{array}{l}\text { Les représentants de l'autorité } \\
\text { dans votre ville encouragent-ils } \\
\text { la vaccination? }\end{array}$} & Religieux & & & & & & & \\
\hline & Oui & $69(22)$ & $29(22)$ & $40(22)$ & \multirow{3}{*}{0,696} & $13(23)$ & $14(27)$ & \\
\hline & Non & $235(75)$ & $105(78)$ & $130(73)$ & & $43(77)$ & $38(73)$ & 0,660 \\
\hline & $N R$ & $8(3)$ & $0(0)$ & $8(4)$ & & $0(0)$ & $0(0)$ & \\
\hline & Politiques & & & & & & & \\
\hline & Oui & $102(33)$ & $41(31)$ & $61(34)$ & & $20(35)$ & $24(46)$ & \\
\hline & Non & $202(65)$ & $93(69)$ & $109(61)$ & 0,696 & $36(64)$ & $28(54)$ & 0,270 \\
\hline & $N R$ & $8(3)$ & $0(0)$ & $8(4)$ & & $0(0)$ & $0(0)$ & \\
\hline & Enseignants & & & & & & & \\
\hline & Oui & $144(46)$ & $64(48)$ & $80(45)$ & & $26(46)$ & $30(57)$ & \\
\hline & Non & $160(51)$ & $70(52)$ & $90(51)$ & 0,903 & $30(54)$ & $22(42)$ & 0,240 \\
\hline & $N R$ & $8(3)$ & $0(0)$ & $8(4)$ & & $0(0)$ & $0(0)$ & \\
\hline & Professionne & de santé & & & & & & \\
\hline & Oui & $224(72)$ & $100(75)$ & $124(70)$ & & $42(75)$ & $39(75)$ & \\
\hline & Non & $80(26)$ & $34(25)$ & $46(26)$ & 0,740 & $14(25)$ & $13(25)$ & 0,990 \\
\hline & $N R$ & $8(2)$ & $0(0)$ & $8(4)$ & & $0(0)$ & $0(0)$ & \\
\hline
\end{tabular}


L'HV selon les différents vaccins du calendrier en vigueur 2019 (annexe 3) est représentée par la figure 2. Le vaccin le plus accepté par les parents était le vaccin combiné contre la diphtérie, le tétanos et la poliomyélite (DTP) associé aux autres valences : haemophilius influenzae (Hib) et coqueluche $(\mathrm{Ca})$. Ils n'observaient aucun refus et un faible taux de retard à l'acceptation : $8 \%, 10 \%$ et $10 \%$ respectivement. En revanche le vaccin qui présentait la plus grande proportion d'HV était celui contre l'hépatite B (VHB) avec 22\% de parents qui ont retardé l'acceptation et $11 \%$ qui ont refusé. En deuxième position, le vaccin contre la grippe saisonnière, non obligatoire en France chez l'enfant sans comorbidité, qui présente 14\% de retard à l'acceptation et $22 \%$ de refus. Suivi du vaccin contre le rotavirus avec $12 \%$ de retard à 1 'acceptation et $20 \%$ de refus. Dans une moindre mesure, le vaccin contre le papillomavirus (HPV) présentait 14\% de retard à 1 'acceptation et $10 \%$ de refus.

Figure 2: Nombre de répondants ayant refusé ou retardé à accepter un vaccin pour leur enfant selon les vaccins du calendrier vaccinal 2019 , dans la population globale $(n=134)$

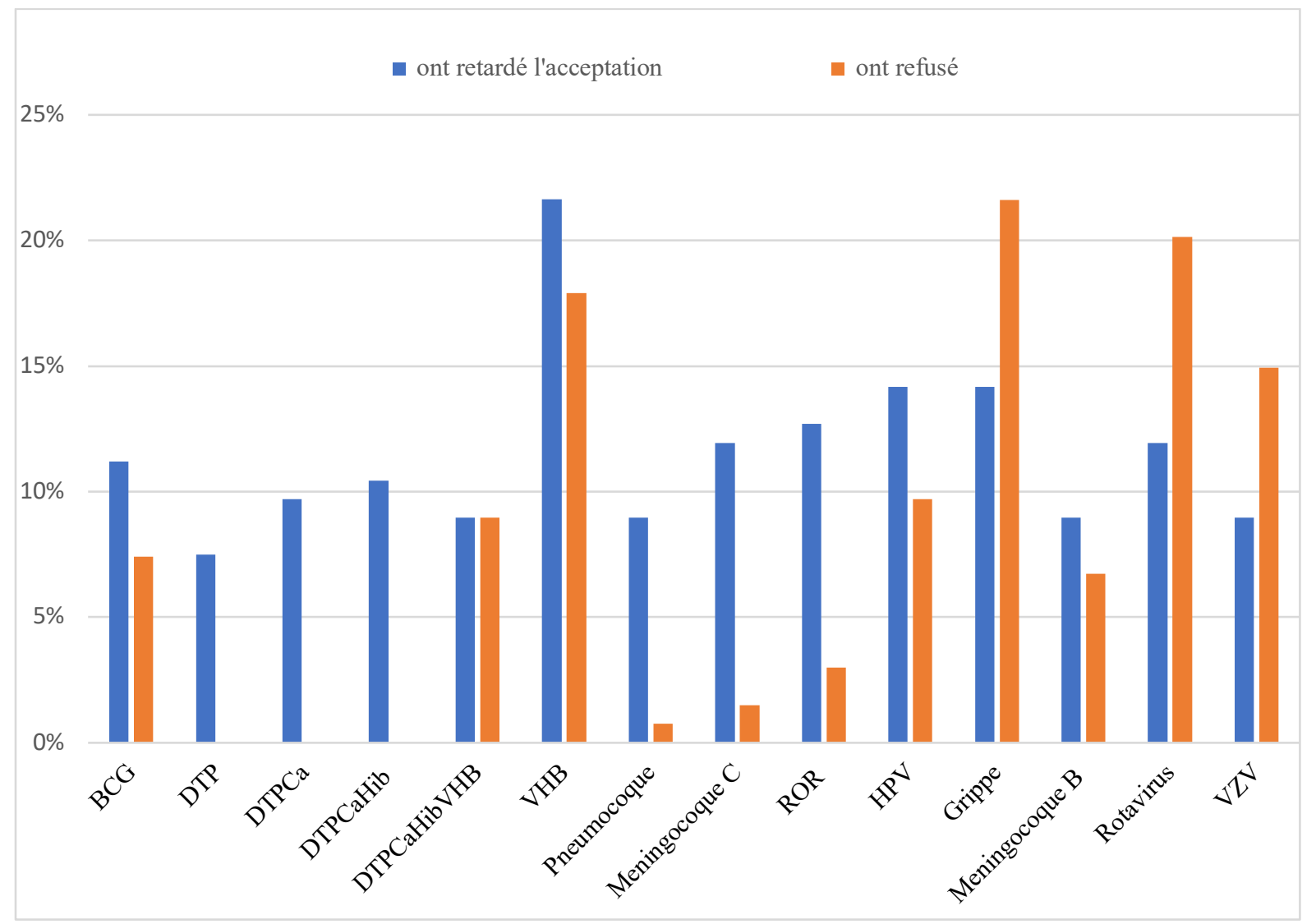

BCG : vaccin bilié de Calmette et Guérin, DTP : vaccin diphtérie -tétanos- polomyélite, Ca : coqueluche, Hib :haemophilius influenzae, VHB : hépatite B, ROR:rubéole-oreillon-rougeole, HPV :papillomavirus:, VZV:varicelle 
Les réponses à la question 32 («Pourquoi avez-vous hésité à faire ce (ou ces) vaccin(s) ou refusé ce (ou ces) vaccin(s) ? ») sont présentées par le tableau 5. Il s'agissait d'une question fermée à choix multiple.

Les préoccupations principales à la vaccination étaient : le manque de sûreté et la peur des effets indésirables (46\%), les informations négatives véhiculées par les médias (27\%) et le manque d'utilité du vaccin (20\%).

Tableau 5 : Les déterminants de l'HV dans la population globale et DA, selon les réponses à la question 32

\begin{tabular}{|c|c|c|c|c|}
\hline \multirow[t]{2}{*}{$\begin{array}{l}\text { Pourquoi avez-vous hésité à faire ce (ou ces) } \\
\text { vaccin(s) ou refusé ce (ou ces) vaccins? }\end{array}$} & $\begin{array}{c}\text { Échantillon } \\
\text { global } \\
\mathbf{N}=134\end{array}$ & $\begin{array}{c}\text { HV } \\
\text { chez les DA } \\
\mathbf{N}=56\end{array}$ & $\begin{array}{c}\text { HV } \\
\text { autres } \\
\text { pathologies } \\
\mathbf{N}=\mathbf{5 2}\end{array}$ & \multirow[t]{2}{*}{$\begin{array}{c}p- \\
\text { value }\end{array}$} \\
\hline & $\mathrm{n}(\%)$ & $\mathrm{n}(\%)$ & $\mathrm{n}(\%)$ & \\
\hline Ce vaccin n'est pas utile & $27(20)$ & $10(18)$ & $17(22)$ & 0,575 \\
\hline Je ne savais pas où aller pour se faire vacciner & $1(1)$ & $1(2)$ & $0(0)$ & 0,236 \\
\hline Je ne pouvais pas quitter mon travail/domicile & $0(0)$ & $0(0)$ & $0(0)$ & l \\
\hline Manque d'information claire et fiable & $16(12)$ & $8(14)$ & $8(10)$ & 0,478 \\
\hline Ce vaccin n'est pas efficace & $6(4)$ & $4(7)$ & $2(3)$ & 0,235 \\
\hline Il n'est pas sur / peur des effets indésirables & $62(46)$ & $27(48)$ & $35(45)$ & 0,702 \\
\hline Informations négatives dans les médias & $36(27)$ & $15(27)$ & $21(27)$ & 0,986 \\
\hline Mauvaise expérience / réaction personnelle & $10(7)$ & $7(13)$ & $3(4)$ & 0,093 \\
\hline Mauvaise réaction autre enfant / connaissance & $13(10)$ & $6(11)$ & $7(9)$ & 0,737 \\
\hline $\begin{array}{l}\text { Mauvaise expérience avec médecin ou structure } \\
\text { de vaccination }\end{array}$ & $3(2)$ & $1(2)$ & $2(3)$ & 0,99 \\
\hline Vaccin dangereux & $19(14)$ & $5(9)$ & $14(18)$ & 0,14 \\
\hline Raisons religieuses & $0(0)$ & $0(0)$ & $0(0)$ & 1 \\
\hline Autres croyances personnelles & $2(1)$ & $1(2)$ & $1(1)$ & 0,999 \\
\hline Peur des aiguilles & $3(2)$ & $1(2)$ & $2(3)$ & 0,999 \\
\hline
\end{tabular}

$D A$ : dermatite atopique, $H V$ : hésitants vaccinaux

Aucun parent n'avait déclaré avoir eu des difficultés à faire vacciner leur enfant à cause de la distance, les horaires d'ouverture, le temps d'attente ou le coût du transport, en rapport à la question 33 . 
Un nombre plus élevé de parents hésitants ont déclaré avoir «d'autres difficultés qui retardent ou ont retardé la vaccination de leurs enfants $»(\mathrm{p}=0,04)$ en réponse à la question 34 , ces raisons sont détaillées dans le tableau 6.

Parmi les $14 \%$ de parents ayant répondus « oui » à la question, la principale raison était la présence d'une contre-indication médicale réelle ou perçue (opération, allergie, maladie grave) (47\%), suivie d'une maladie bégnine présentée par l'enfant (virose ou fièvre) (21\%).

Tableau 6 : Autres raisons qui retardent ou ont retardé la vaccination dans la population globale et DA, selon les réponses à la question 34

\begin{tabular}{|c|c|c|c|c|c|c|c|c|c|c|}
\hline \multirow{3}{*}{$\begin{array}{l}\text { Existe-t-il d'autres } \\
\text { difficultés dans votre } \\
\text { vie qui retardent ou } \\
\text { ont retardé la } \\
\text { vaccination de vos } \\
\text { enfants? }\end{array}$} & \multirow{2}{*}{\multicolumn{2}{|c|}{$\begin{array}{c}\text { Échantillon } \\
\text { global } \\
\mathbf{N}=\mathbf{3 1 2}\end{array}$}} & \multicolumn{2}{|c|}{ Population } & \multirow{3}{*}{$\begin{array}{c}p- \\
\text { value }\end{array}$} & \multirow{2}{*}{\multicolumn{2}{|c|}{$\underset{\mathbf{N}=134}{\mathbf{H V}}$}} & \multirow{2}{*}{\multicolumn{2}{|c|}{$\begin{array}{c}\text { Non HV } \\
\mathbf{N}=\mathbf{1 7 8}\end{array}$}} & \multirow{3}{*}{$\begin{array}{c}p- \\
\text { value }\end{array}$} \\
\hline & & & $\begin{array}{l}\text { DA } \\
N=108\end{array}$ & $\begin{array}{l}\text { Autres } \\
N=176\end{array}$ & & & & & & \\
\hline & $\mathrm{n}$ & $\%$ & $\mathrm{n}(\%)$ & $\mathrm{n}(\%)$ & & $\mathrm{n}$ & $\%$ & $\mathrm{n}$ & $\%$ & \\
\hline Oui & 27 & 9 & $15(14)$ & $12(6)$ & \multirow{3}{*}{0,496} & 19 & 14 & 8 & 5 & \multirow{3}{*}{$0,004^{*}$} \\
\hline Non & 278 & 91 & $93(86)$ & $185(94)$ & & 115 & 86 & 163 & 95 & \\
\hline NR & 7 & 2 & $0(0)$ & $7(3)$ & & 0 & 0 & 7 & 4 & \\
\hline $\begin{array}{l}\text { Contre-indication } \\
\text { médicale réelle ou } \\
\text { perçue (opération, } \\
\text { allergie, maladie grave) }\end{array}$ & 9 & 33 & 4 & 5 & - & 9 & 47 & 0 & 0 & - \\
\hline $\begin{array}{l}\text { Maladie bégnine de } \\
\text { l'enfant }\end{array}$ & 6 & 22 & 2 & 4 & - & 4 & 21 & 2 & 25 & - \\
\hline $\begin{array}{l}\text { Rupture de stock du } \\
\text { vaccin }\end{array}$ & 4 & 15 & 4 & 0 & - & 0 & 0 & 4 & 50 & - \\
\hline Oubli des rappels & 2 & 7 & 1 & 1 & - & 2 & 11 & 0 & 0 & - \\
\hline $\begin{array}{l}\text { Non disponibilité du } \\
\text { médecin }\end{array}$ & 2 & 7 & 2 & 0 & - & 1 & 5 & 1 & 13 & - \\
\hline $\begin{array}{l}\text { Évènements de vie } \\
\text { (conflit dans le couple) }\end{array}$ & 1 & 4 & 0 & 1 & - & 1 & 5 & 0 & 0 & - \\
\hline
\end{tabular}

$D A$ : dermatite atopique, $H V$ : hésitants vaccinaux, $N R$ : non répondu 
D’après les réponses à la question 35 ("Pensez-vous qu'il y ait d'autres raisons pour lesquelles les enfants ne devraient pas être vaccinés?»),10\% des répondants avait répondu « oui ». Ils étaient constitués pour la quasi-totalité de parents hésitants $(20 \%, p<0,001)$. Les raisons détaillées par les parents sont représentées par le tableau 7 et organisées selon les déterminants de l'HV par l'OMS (annexe 2). On retrouve en première ligne une balance bénéfice/risque en défaveur de la vaccination par leurs « effets secondaires à long terme », et le « déclenchement de maladies » $(37 \%)$ suivi du « rôle des professionnels de santé » $(23 \%)$ et de la peur des nouveaux vaccins mentionnée par 13\% des hésitants.

Tableau 7 : Autres raisons pour lesquelles les enfants ne devraient pas être vaccinés dans la population globale, selon les réponses à la question 35

\begin{tabular}{|c|c|c|c|c|c|c|c|}
\hline \multirow{2}{*}{$\begin{array}{l}\text { Pensez-vous qu'il y ait d'autres raisons pour lesquelles les enfants } \\
\text { ne devraient pas être vaccinés? }\end{array}$} & \multicolumn{2}{|c|}{$\begin{array}{l}\text { global } \\
\mathbf{N}=312\end{array}$} & \multicolumn{2}{|c|}{$\mathrm{N}=134$} & \multicolumn{2}{|c|}{$N=178$} & \\
\hline & n & $\%$ & n & $\%$ & $\mathbf{n}$ & $\%$ & \\
\hline Oui & 31 & 10 & 27 & 20 & 4 & 2 & \\
\hline Non & 274 & 88 & 107 & 80 & 167 & 94 & $<0,001 *$ \\
\hline NR & 7 & 2 & 0 & 0 & 7 & 4 & \\
\hline
\end{tabular}

\begin{tabular}{|c|c|c|c|c|c|c|}
\hline \multicolumn{7}{|l|}{ Influence Contextuelle : } \\
\hline Rôles des politiques vaccinales (majoration des statistiques) & 1 & 3 & 1 & 4 & 0 & 0 \\
\hline Rôle de l'industrie pharmaceutique (intérêt financier) & 3 & 10 & 3 & 12 & 0 & 0 \\
\hline \multicolumn{7}{|l|}{ Influence Individuelle : } \\
\hline Antécédents vaccinaux (allergie) & 3 & 10 & 3 & 12 & 0 & 0 \\
\hline Croyances et attitudes face à la santé et à la prévention & 2 & 7 & 2 & 8 & 0 & 0 \\
\hline Sur vaccination des enfants & 1 & - & - & - & - & - \\
\hline Laisser le système immunitaire se construire naturellement & 1 & - & - & - & - & - \\
\hline Rapport bénéfice/risque (perçu, heuristique) & 12 & 39 & 10 & 37 & 2 & 50 \\
\hline Peur des effets secondaires futurs & 7 & - & - & - & - & - \\
\hline Déclenchement de maladie & 3 & - & - & - & - & - \\
\hline Infection aigue/maladie bénigne de l'enfant & 2 & - & - & - & - & - \\
\hline \multicolumn{7}{|l|}{ Spécificité vaccinale : } \\
\hline Introduction d'un nouveau vaccin & 4 & 13 & 4 & 15 & & \\
\hline Manque de recul & 1 & - & - & - & & \\
\hline Vaccin non obligatoire & 3 & - & - & - & & \\
\hline Le rôle des professionnels de santé & 8 & 27 & 6 & 23 & 2 & 50 \\
\hline CI maladie grave/rare & 4 & - & - & - & - & - \\
\hline CI prédisposition familiale & 1 & - & - & - & - & - \\
\hline Non proposé par le médecin & 1 & - & - & - & - & - \\
\hline Refus du médecin & 2 & - & - & - & - & - \\
\hline
\end{tabular}

$H V$ : hésitants vaccinaux, $N R$ : non répondu 
Parmi les 312 parents répondants, plus de la moitié (64\%) déclaraient avoir déjà reçu ou entendu des informations négatives au sujet de la vaccination.

L'influence de ces informations dites négatives était prépondérante chez les parents hésitants avec $79 \%$ de réponses positives contre $53 \%$ chez les non hésitants $(p<0,001)$. Ce lien persistait après ajustement sur d'autres facteurs.

Au total, seulement $78 \%$ des hésitants avaient quand même vacciné leurs enfants malgré avoir reçu ces informations (contre 95\% de non hésitants, $p<0,001$ ).

Cela signifie que moins d'un tiers des parents hésitants vaccinent leurs enfants après avoir entendu des informations négatives $(21 \%)$.

Le détail des réponses est représenté par le tableau 8. La plupart des informations négatives reçues par les parents hésitants concernaient le rapport bénéfice/risque perçu de façon individuel (29) comme les « effets secondaires à long terme », les « effets indésirables graves », et le fait que les « vaccins sont dangereux pour la santé », mais également l'impact des controverses et polémiques sur certains vaccins comme la « sclérose en plaque et VHB », « autisme et ROR », « handicap neurologique à long terme » (49), diffusée en grande partie par les médias (10). 
Tableau 8 : Informations négatives reçues ou entendues au sujet des vaccins selon les réponses à la question 36

\begin{tabular}{|c|c|c|c|c|c|c|c|}
\hline \multirow{2}{*}{$\begin{array}{l}\text { Avez-vous déjà reçu ou entendu des informations négatives } \\
\text { au sujet des vaccins? }\end{array}$} & \multicolumn{2}{|c|}{$\begin{array}{c}\text { Échantillon } \\
\text { global } \\
\mathbf{N}=312 \\
\end{array}$} & \multicolumn{2}{|c|}{$\begin{array}{c}\text { HV } \\
\mathbf{N}=134\end{array}$} & \multicolumn{2}{|c|}{$\begin{array}{c}\text { Non HV } \\
\mathbf{N}=178\end{array}$} & \multirow{2}{*}{ p-value } \\
\hline & $\mathbf{n}$ & $\%$ & $\mathbf{n}$ & $\%$ & $\mathbf{n}$ & $\%$ & \\
\hline Oui & 201 & 64 & 106 & 79 & 95 & 53 & \multirow{3}{*}{$<0,001^{*}$} \\
\hline Non & 103 & 33 & 28 & 21 & 75 & 42 & \\
\hline NR & 8 & 3 & 0 & 0 & 8 & 5 & \\
\hline
\end{tabular}

Avez-vous quand même vacciné vos enfants après avoir reçu cette information?

\begin{tabular}{|c|c|c|c|c|c|c|c|}
\hline Oui & 173 & 86 & 83 & 78 & 90 & 95 & \multirow{2}{*}{$<0,001^{*}$} \\
\hline Non & 28 & 14 & 23 & 22 & 5 & 5 & \\
\hline \multicolumn{8}{|l|}{ Nature des informations négatives : } \\
\hline \multicolumn{8}{|l|}{ Influence Contextuelle : } \\
\hline Communication et médias & 18 & & 10 & & 8 & & \\
\hline Rôle de l'industrie pharmaceutique (intérêt financier) & 1 & & 0 & & 1 & & \\
\hline \multicolumn{8}{|l|}{ Influence Individuelle : } \\
\hline $\begin{array}{l}\text { Croyances et attitudes face à la santé et à la prévention } \\
\text { (immunité) }\end{array}$ & 2 & & 0 & & 2 & & \\
\hline Laisser le système immunitaire se construire naturellement & 2 & & 0 & & 2 & & \\
\hline Rapport bénéfice/risque (perçu, heuristique) & 53 & & 29 & & 24 & & \\
\hline Effets secondaires à long terme & 26 & & 16 & & 10 & & \\
\hline Effets indésirables graves (malaise, décès) & 5 & & 1 & & 4 & & \\
\hline Vaccins dangereux pour la santé & 13 & & 11 & & 2 & & \\
\hline Vaccin inutile/inefficace & 7 & & 0 & & 7 & & \\
\hline L'immunisation comme norme sociale & 3 & & 2 & & 1 & & \\
\hline \multicolumn{8}{|l|}{ Spécificité vaccinale : } \\
\hline Rapport bénéfice/risque (données scientifiques) & 73 & & 49 & & 24 & & \\
\hline Allergie & 2 & & 2 & & 0 & & \\
\hline$V H B$ & 9 & & 1 & & 8 & & \\
\hline Sclérose en plaque & 1 & & 1 & & 0 & & \\
\hline Sclérose en plaque et $V H B$ & 12 & & 9 & & 3 & & \\
\hline Autisme et ROR & 8 & & 4 & & 4 & & \\
\hline Syndrome de Guillain Barré et Grippe & 1 & & 1 & & 0 & & \\
\hline Handicap neurologique au long terme & 8 & & 3 & & 5 & & \\
\hline Composition toxique (adjuvant) & 8 & & 6 & & 2 & & \\
\hline \multicolumn{8}{|l|}{ Introduction d'un nouveau vaccin } \\
\hline Manque de recul vaccin $H P V$ & 7 & & 5 & & 2 & & \\
\hline Rotavirus & 8 & & 8 & & 0 & & \\
\hline Grippe saisonnière & 9 & & 9 & & 0 & & \\
\hline Le rôle des professionnels de santé & 2 & & 2 & & 0 & & \\
\hline
\end{tabular}


Concernant la question 37 ("Les représentants de l'autorité dans votre ville encouragent-ils la vaccination?»), les parents ont répondu en quasi-totalité que les professionnels de santé encouragent la vaccination (74\%). Aucune différence significative a été mise en évidence entre les populations.

Une régression logistique modélisant l'HV dans la population globale est représentée par le tableau 9. Cela confirmait que l'HV était associée à la DA de façon significative : $p=0,037$ (OR $1,78 ;$ IC $95[1,06 ; 3,05])$.

Les parents de filles étaient plus hésitants ( $p=0,027$, OR 0,55; IC95 [0,33;0,93]).

La profession des parents était également associée de façon significative au fait d'être hésitant $(p=0,001)$, ainsi ceux qui exerçaient en tant qu'artisans/commerçants/agriculteurs/chefs d'entreprise étaient plus hésitants que les employés/ouvriers ( $p=0,015$, OR 3,30 ; IC95 [1,24 ; $8,77])$. Les cadres/professions intermédiaires était une catégorie socio-professionnelle moins hésitante $(\mathrm{p}=0,008$, OR 0,$43 ;$ IC95 $[0,23 ; 0,78])$.

Penser que les vaccins protègent des maladies graves diminue le risque d'être hésitant vaccinal $(\mathrm{p}<0,001$, OR 0,203; IC95 [0,085;0,486]). L'influence des informations dites négatives était prépondérante chez les parents dits hésitants (p<0,001, OR 4,00; IC95 [2,20; 7,26]).

Tableau 9: Déterminants de l'HV dans la population globale après régression logistique (OR ajustés) $(\mathrm{n}=312)$

\begin{tabular}{|c|c|c|}
\hline & p-value & OR [IC-95\%] \\
\hline DA & $0,037 *$ & $1,78[1,06 ; 3,05]$ \\
\hline Sexe enfant : Fille & $0,027 *$ & $0,55[0,33 ; 0,93]$ \\
\hline Profession parent & $0,001 *$ & \\
\hline Cadre / profession intermédiaire versus Employé / ouvrier & $0,008^{*}$ & $0,43[0,23 ; 0,78]$ \\
\hline Artisans / commerçants / agriculteurs / chef d'entreprise versus Employé / ouvrier & $0,015^{*}$ & $3,30[1,24 ; 8,77]$ \\
\hline Retraités versus Employé / ouvrier & 0,875 & $0,95[0,47 ; 1,92]$ \\
\hline Pense que les vaccins protègent des maladies graves & $<0,001^{*}$ & $0,203[0,09 ; 0,49]$ \\
\hline Avez-vous déjà reçu ou entendu des informations négatives sur les vaccins? & $<0,001^{*}$ & $4[2,20 ; 7,26]$ \\
\hline
\end{tabular}

$D A$ : dermatite atopique 


\section{b) Hésitation vaccinale et ses déterminants dans la population DA}

Les caractéristiques et déterminants de l'HV dans la population DA sont représentées par les tableaux 3 et 4 .

Les caractéristiques propres de l'enfant atteint de DA, sont représentées par le tableau 11.

Les enfants atteints de DA souffraient à $46 \%$ d'une autre pathologie du terrain atopique, principalement d'asthme (30\%), d'allergie alimentaire (26\%), de rhinite (19\%) et de conjonctivite allergique (12\%). Seulement $22 \%$ avait un frère ou une sœur atteinte de DA. Il n’y avait pas de différence significative entre les hésitants et non hésitants.

Tableau 10 : Déterminants de l'HV spécifiques de la population DA

\begin{tabular}{|c|c|c|c|c|c|}
\hline & & $\underset{\substack{\text { DA } \\
\mathbf{N}=108}}{ }$ & $\begin{array}{c}\text { HV chez } \\
\text { DA } \\
\mathbf{N}=\mathbf{5 6}\end{array}$ & $\begin{array}{c}\text { Non HV } \\
\text { DA } \\
\mathbf{N}=\mathbf{5 2} \\
\end{array}$ & p-value \\
\hline & & $n(\%)$ & $n(\%)$ & $n(\%)$ & \\
\hline \multirow{3}{*}{ Autre(s) maladie(s) associée(s) } & Oui & $50(46)$ & $24(44)$ & $26(51)$ & \multirow{3}{*}{0,503} \\
\hline & Non & $55(51)$ & $30(56)$ & $25(49)$ & \\
\hline & $N R$ & $3(1)$ & $2(3)$ & $1(2)$ & \\
\hline \multirow{4}{*}{$\begin{array}{l}\text { Si oui, autres pathologies du terrain } \\
\text { atopique }\end{array}$} & Asthme & $32(30)$ & $16(29)$ & $16(31)$ & 0,578 \\
\hline & Allergie alimentaire & $28(26)$ & $12(21)$ & $16(31)$ & 0,268 \\
\hline & Rhinite allergique & $20(19)$ & $14(25)$ & $6(12)$ & 0,072 \\
\hline & $\begin{array}{l}\text { Conjonctivite } \\
\text { allergique }\end{array}$ & $13(12)$ & $7(13)$ & $6(12)$ & 0,878 \\
\hline \multirow{2}{*}{ Autres DA dans la fratrie } & Oui & $24(22)$ & $12(21)$ & $12(23)$ & \multirow{2}{*}{0,837} \\
\hline & Non & $84(78)$ & $44(79)$ & $40(77)$ & \\
\hline \multirow{2}{*}{ Participation à un ETP } & Oui & 21(19) & $12(21)$ & $9(17)$ & \multirow{2}{*}{0,589} \\
\hline & Non & $87(81)$ & $44(79)$ & $43(83)$ & \\
\hline
\end{tabular}

$D A$ : dermatite atopique, $H V$ : hésitants vaccinaux, ETP : éducation thérapeutique du patient 
Les parents hésitants vaccinaux d'enfants atteints de DA étaient pour moitié des employés/ouvriers (52\%). Les cadres / professions intermédiaires et retraités étaient beaucoup moins représentés (18\% versus 35\%, $\mathrm{p}=0,003)$ (tableau 3 ).

Ce lien persistait après ajustement sur d'autres facteurs. L'analyse multivariée est représentée par le tableau 10.

La profession des parents était significativement associée au fait d'être hésitant ou non ( $\mathrm{p}=0,001)$ avec une hésitation plus forte chez les employés/ouvriers que chez les retraités/sans activité professionnel/autre $\mathrm{p}=0,005$ (OR 0,16; IC95 [0,04;0,57]). Le fait que l'enfant présente une autre pathologie atopique majorait l'HV chez les parents, en particulier s'il était atteint d'une rhinite allergique $\mathrm{p}=0,017$ (OR 4,82; IC95[1,33; 17,4]), à la différence de ceux dont l'enfant était atteint d'une allergie alimentaire qui étaient moins hésitants $p=0,012$ (OR 0,24 ; IC95 $[0,08 ; 0,73])($ tableau11)

Tableau 11 : Déterminants de 1'HV dans la population DA après régression logistique (OR ajustés) $(\mathrm{n}=108)$

\section{p-value OR [IC-95\%]}

\begin{tabular}{|c|c|c|}
\hline Allergie alimentaire & $0,012 *$ & $0,24[0,08 ; 0,73]$ \\
\hline Rhinite allergique & $0,017^{*}$ & $4,82[1,33 ; 17,4]$ \\
\hline Profession & $0,001^{*}$ & \\
\hline Cadre / profession intermédiaire versus Employé / ouvrier & 0,09 & $0,24[0,08 ; 0,69]$ \\
\hline $\begin{array}{l}\text { Artisans / commerçants / agriculteurs / chef d'entreprise } \\
\text { versus Employé / ouvrier }\end{array}$ & 0,168 & $3,29[0,60 ; 17,9]$ \\
\hline Retraités versus Employé / ouvrier & $0,05^{*}$ & $0,16[0,04 ; 0,57]$ \\
\hline
\end{tabular}


Les principaux vaccins pour lesquels les parents étaient le plus réticents sont représentés par la figure 3. Ils s'agissaient des mêmes vaccins que les hésitants dans la population globale avec une proportion plus élevée de retard à l'acceptation concernant le vaccin antiHPV $(20 \%)$ et de refus $(11 \%)$ contre $10 \%$ et $9 \%$ respectivement dans les autres pathologies dermatologiques.

Figure 3: Nombre de répondants ayant refusé ou retardé à accepter un vaccin pour leur enfant selon les vaccins du calendrier vaccinal 2019 dans la population DA $(n=108)$

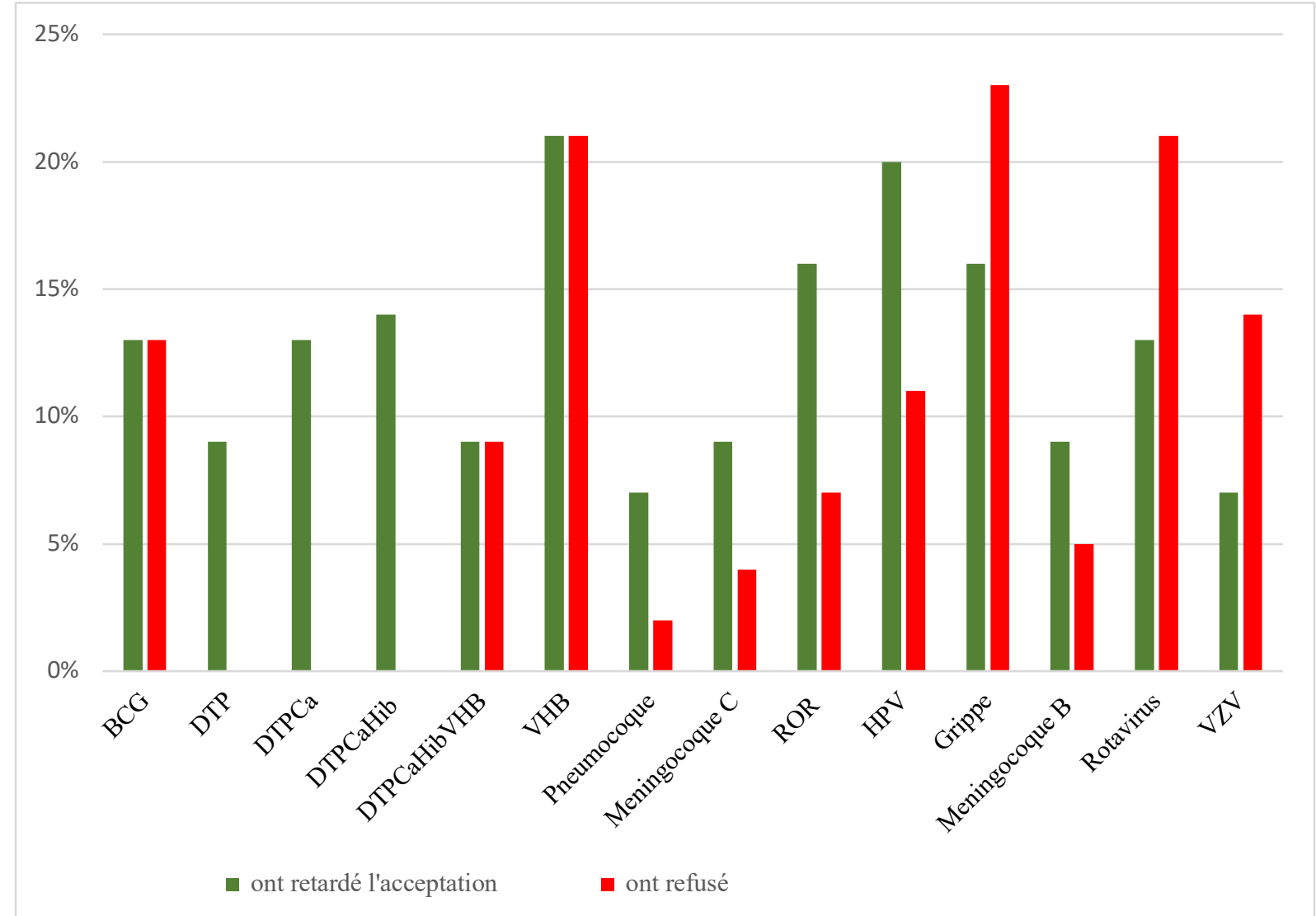

BCG : vaccin bilié de Calmette et Guérin, DTP : vaccin diphtérie -tétanos- polomyélite, Ca : coqueluche, Hib : Haemophilus influenzae, VHB : hépatite B, ROR:rubéole-oreillon-rougeole, HPV :papillomavirus:, VZV:varicelle 
Les principales préoccupations à la vaccination chez les hésitants vaccinaux, en réponse à la question 32 ("Pourquoi avez-vous hésité à faire ce (ou ces) vaccin(s) ou refusé ce (ou ces) vaccins? »), sont représentées par le tableau 5 et repris par la figure 4. Les causes étaient similaires à celles retrouvées dans la population globale. Nous notons une différence sur le fait que plus de parents d'enfants souffrant de DA ont eu une mauvaise expérience ou réaction avec un vaccin chez leur enfant ( $13 \%$ versus $4 \%, p=0,0093)$.

Figure 4 : Déterminants de 1'HV dans la population DA, selon les réponses de la question 32 $(\mathrm{N}=134)$

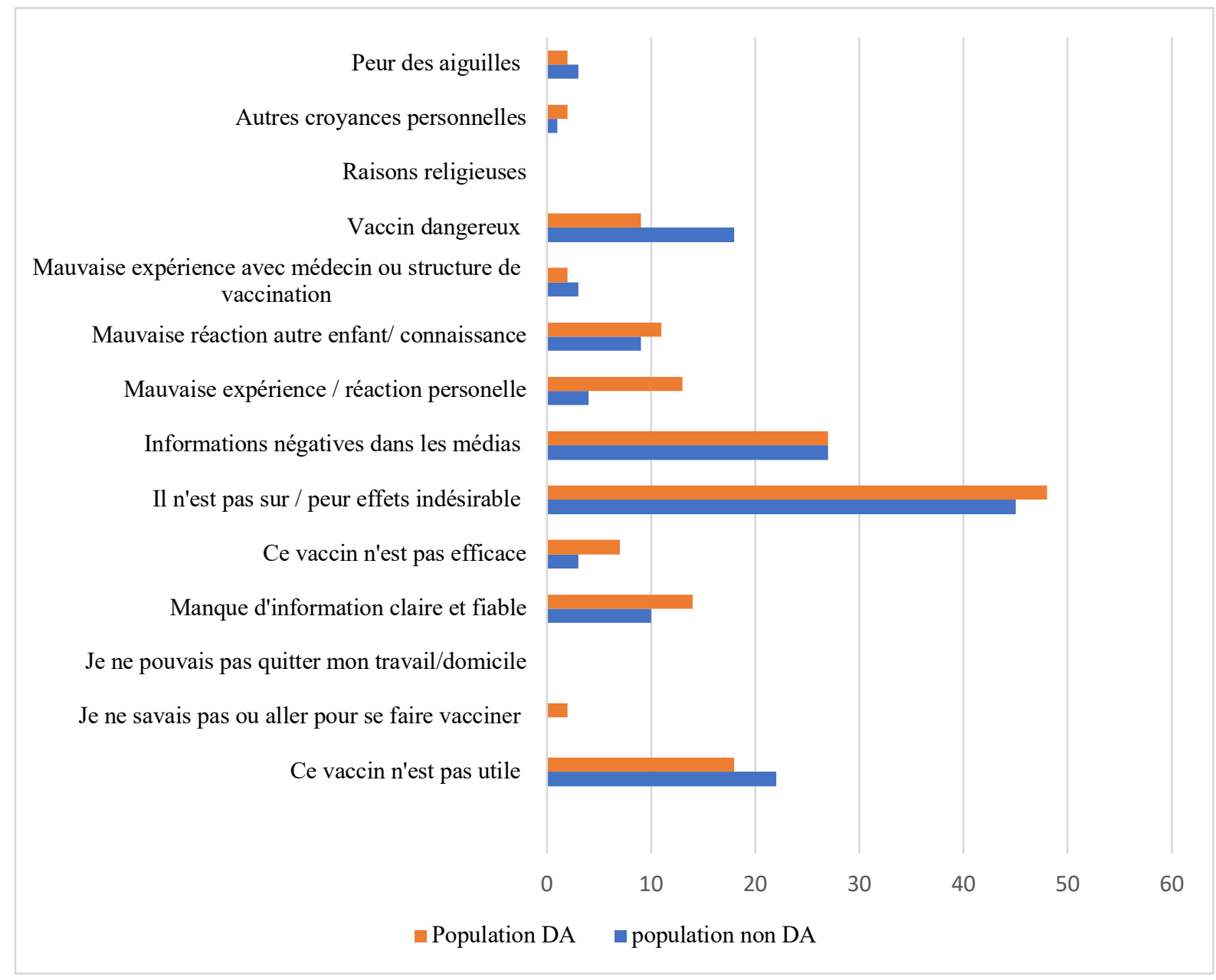

$D A$ : dermatite atopique

Concernant les autres questions évaluant les déterminants de l'HV dans la population DA, elles étaient similaires à celles de la population globale. Il n'y avait donc pas de différence significative sur les autres déterminants de l'HV dans cette population. 


\section{3) Lien entre l'HV et la corticophobie dans la population DA}

On notait plus de craintes ou réticences vis-à-vis des dermocorticoïdes chez les parents d'enfants atteints de DA (tableau 12). Cependant, il y a beaucoup de données manquantes dans la population d'enfants atteints d'autres pathologies dermatologiques, probablement expliquées par le fait que cette population ne se sente pas concernée devant le peu d'expérience sur les dermocorticoïdes (13\% seulement ont eu une prescription de crèmes à base de cortisone plus d'une 1 semaine). Ces résultats sont donc difficilement interprétables.

Concernant les hésitants vaccinaux avec un enfant atteint de DA, ils avaient tendance à avoir plus de craintes et de réticences à utiliser des crèmes à base de cortisone que les non-hésitants $(\mathrm{p}=0,053)$ (tableau 12).

Tableau 12: Attitudes des parents sur les crèmes à base de cortisone dans la population DA

\begin{tabular}{|c|c|c|c|c|c|c|c|}
\hline & & $\begin{array}{c}\text { Échantillon } \\
\text { DA } \\
\mathbf{N}=\mathbf{1 0 8}\end{array}$ & $\begin{array}{c}\text { Autres } \\
\text { pathologies } \\
\text { dermato } \\
\mathbf{N}=\mathbf{2 0 4}\end{array}$ & p-value & $\begin{array}{c}\text { HV chez } \\
\text { DA } \\
\mathbf{N}=\mathbf{5 6}\end{array}$ & $\begin{array}{c}\text { Non HV } \\
\text { DA } \\
\mathbf{N}=52\end{array}$ & p-value \\
\hline & & n(\%) & $\mathbf{n}(\%)$ & & $\mathbf{n}(\%)$ & $\mathbf{n}(\%)$ & \\
\hline \multirow{3}{*}{$\begin{array}{l}\text { Craintes / réticences } \\
\text { crèmes corticoïdes }\end{array}$} & Oui & $58(54)$ & $35(22)$ & \multirow{3}{*}{$<0,001$} & $35(63)$ & $23(44)$ & \multirow{3}{*}{0,053} \\
\hline & Non & $50(46)$ & $121(78)$ & & $21(37)$ & $29(56)$ & \\
\hline & NR & $0(0)$ & $48(23)$ & & $0(0)$ & $0(0)$ & \\
\hline \multirow{3}{*}{$\begin{array}{l}\text { Crèmes corticoïdes > } \\
1 \text { semaine }\end{array}$} & Oui & $95(88)$ & $27(13)$ & \multirow{3}{*}{$<0,001$} & - & - & \\
\hline & Non & $13(12)$ & $175(86)$ & & - & - & \\
\hline & NR & $0(0)$ & $2(1)$ & & - & - & \\
\hline
\end{tabular}


Notre étude retrouvait un score global de corticophobie, évaluée avec le score TOPICOP, de $41 \%$ chez les parents d'enfants atteints de DA. Il n'y avait pas de lien significatif entre la corticophobie globale et l'HV, par contre il y avait un lien entre les fausses croyances concernant les dermocorticoïdes et l'hésitation à vacciner ( $40 \%$ versus $32 \%, \mathrm{p}=0,048)$ (tableau 13).

$\underline{\text { Tableau } 13}$ : Score TOPICOP moyen $(\%)$ dans la population DA $(\mathrm{n}=108)$

Score TOPICOP moyen \% Échantillon DA HV chez DA Non HV DA p-value

\begin{tabular}{ccccc}
\hline Croyances & 36 & 40 & 32 & $0,048^{*}$ \\
\hline Peurs & 47 & 49 & 44 & 0,267 \\
\hline Global & 41 & 45 & 38 & 0,105 \\
\hline
\end{tabular}

$D A$ : dermatite atopique, $H V$ : hésitants vaccinaux

Le score TOPICOP a été évalué initialement dans notre questionnaire chez tous les parents d'enfants souffrant aussi bien de DA que d'une autre pathologie dermatologique. Le nombre de données manquantes dans la population de parents d'enfants non atteints de DA était conséquente (54 non-réponses). Nous avons donc décidé de ne pas analyser le score TOPICOP dans cette catégorie de parents, probablement moins concernés que les parents d'enfants atteints de DA. 


\section{DISCUSSION}

\section{1) Résultats principaux}

L'objectif de notre travail était d'évaluer l'HV des parents d'enfants atteints de DA par rapport aux autres pathologies dermatologiques, d'en définir les déterminants et de rechercher un lien avec la corticophobie dans cette population de parents d'enfants atteints de DA. $\mathrm{Au}$ terme de notre travail et sur la base d'un nombre conséquent de réponses ( $\mathrm{n}=312$ ), nous avons mis en évidence un risque plus élevé d'être hésitant à l'égard de la vaccination dans les familles d'enfants atteints de DA ( $52 \%$ versus $38 \%, p=0,0021)$. Ce lien persistait après ajustement sur d'autres facteurs ( $p=0,037$, OR 1,78 ; IC $95[1,06 ; 3,05])$. Cette hésitation était encore plus marquée si les enfants souffraient d'une autre comorbidité atopique comme une une rhinite allergique $(\mathrm{p}=0,017,4,82[1,33 ; 17,4])$.

A notre connaissance, c'est la première étude ayant évalué les attitudes et pratiques en matière de vaccination chez les parents d'enfants atteints de DA avec un outil récemment développé et validé par l'OMS tel que le questionnaire VHS, et à mettre en évidence un surrisque d'HV dans cette population.

Cette proportion d'hésitants vaccinaux semble d'autant plus inquiétante qu'elle a pour conséquence un retard vaccinal chez ces enfants. En effet seulement $57 \%$ des parents hésitants d'enfants atteints de DA déclaraient être «à jour » des vaccins de leurs enfants contre $83 \%(\mathrm{p}=0,009)$.

Leurs principales réticences portaient sur les vaccins contre l'hépatite B (VHB), la grippe saisonnière et le rotavirus probablement perçues, à tort, comme maladies « bénignes » par cette catégorie de parents. En effet nous avons démontré que le fait de croire que « les vaccins protègent des maladies graves » diminuait le risque d'être hésitant ( $\mathrm{p}<0,001$, OR 0,203 ; IC95 $[0,085 ; 0,486])$. 
Les raisons évoquées de cette HV concernaient en très grande partie la sécurité du produit et la peur d'effets indésirables (45\%), la proportion d'informations négatives relayées par les médias (22\%), et leur manque d'utilité (18\%). On notait une proportion plus élevée de mauvaises expériences et réactions dans le passé chez les parents d'enfants atteints de DA $(13 \%$ contre $4 \%)$.

Nous avons également établi un lien entre la catégorie socio-professionnelle des parents et leur hésitation à faire vacciner leurs enfants dans la population DA avec une proportion plus importante chez les employés/ouvriers $(\mathrm{p}=0,001)$ et moindre chez les cadres/professions intermédiaires ( $\mathrm{p}=0,009$, OR 0,24; IC95 [0,08;0,69]).

La part des controverses et polémiques au sujet des principaux vaccins était prépondérante dans les réponses ouvertes des parents concernant les informations négatives reçues ou entendues. De même qu'une balance bénéfice/risque en défaveur de la vaccination était le plus souvent rapportée comme autre raison pour laquelle les enfants ne devaient pas être vaccinés. En effet le fait d'avoir entendu des informations négatives à ce sujet augmentait l'HV (p<0,001, OR 4 ; IC95 [2,20;7,26]).

$\mathrm{Au}$ terme de notre travail, nous avons pu remarquer que les déterminants qui marquaient l'HV chez les parents d'enfants atteints de DA étaient les mêmes que ceux de notre population générale.

Les professionnels de santé représentaient en grande majorité l'autorité qui encourage le plus la vaccination (74\%). Ces résultats confirment le rôle crucial des professionnels de santé pour influencer le choix des parents en matière de vaccination.

Notre travail nous a permis également de confirmer que les parents d'enfants atteints de DA étaient plus corticophobes et que ceux qui hésitaient à faire vacciner leurs enfants avaient tendance à avoir plus de craintes ou réticences à l'application de dermocorticoïdes (63\% versus $44 \%, p=0,053)$, avec un score de corticophobie plus élevé sur les fausses croyances (40\% versus $32 \%, p=0,048)$. Ces constatations mettent en lumière un lien entre cette crainte excessive des dermocorticoïdes et celle de faire vacciner son enfant jusque-là jamais exploré à notre connaissance. 


\section{2) Confrontation aux données de la littérature}

Dans notre échantillon, $43 \%$ des répondeurs hésitaient à faire vacciner leurs enfants, nos résultats concordent avec ceux de l'étude de Larson et al. qui retrouvait un plus grand scepticisme à l'égard des vaccins en France avec $41 \%$ de réponses négative à la question « Je pense que les vaccins sont sûrs » contre 13\% dans le reste du monde. Cette étude portait sur environ 66000 personnes interrogées dans 67 pays différents (10). Cette variation peut être due aux différentes populations étudiées dans différents contextes (c'est-à-dire zones urbaines contre zones rurales, pays développés contre pays en développement, pays pratiquant des calendriers de vaccination différents).

Concernant les études portant sur une population plus proche de la nôtre à savoir sur des parents d'enfants de moins de 18 ans dans des pays uniquement développés, nos résultats concordent avec deux enquêtes menées respectivement au Canada en 2014 et Australie en 2012. Selon ces enquêtes, $40 \%$ et $52 \%$, respectivement, ont déclaré avoir des doutes quant à la vaccination de leur enfant (34)(35). Les différences entre les études concernant la définition de l'HV et les populations étudiées rendent les comparaisons difficiles.

Le statut vaccinal des enfants de parents hésitants était moins «à jour» que ceux qui n'hésitaient pas $(61 \%$ contre $79 \%, \mathrm{p}<0,001)$ avec une part plus importante pour les vaccins « uniquement obligatoires » $(25 \%$ versus $7 \%, \mathrm{p}<0,001)$. Ces chiffres sont bien en deçà de l'objectif fixé par la loi relative à la politique de santé publique qui est d'obtenir une couverture vaccinale de $>95 \%$ pour une immunité de groupe (36). Les préoccupations à l'égard des vaccins contribuent donc à une sous immunisation des enfants, ceci a été rapporté dans l'étude américaine de Gust DA et al. qui compare les ménages avec des enfants sous immunisés sur au moins deux vaccins avec ceux ayant un statut vaccinal à jour (37). L'étude canadienne de Dubbé E et al. le confirme avec $58 \%$ d'enfants « à jour » chez les parents hésitants contre $95,3 \%$ chez les parents non hésitants $(\mathrm{p}<0,001)$ (34). Le statut vaccinal peut donc être la conséquence d'attitudes, de croyances et de comportements néfastes dont il faut se préoccuper. Les personnes peuvent être entièrement vaccinées mais avoir des inquiétudes quant à la vaccination, c'est ce qu'Enkel et al. ont décrit en 2018 comme des « hésitants compliants » (38). 
L'adhésion envers la vaccination est un phénomène complexe mettant en jeu plusieurs déterminants sociodémographiques à prendre en compte pour lever les réticences et redonner confiance dans la vaccination (7).

Dans notre étude, la répartition des catégories socio professionnelles était représentative de la population française (39).

La profession était liée significativement au statut d'hésitant vaccinal $(p<0,001)$. Les parents exerçant en tant qu'ouvriers/employés étaient plus préoccupés et plus sceptiques à l'égard de la vaccination que les cadres et professions intermédiaires $(p=0,008)$ et étaient donc plus susceptibles de demander des exemptions. En revanche nous n'avons pas trouvé de lien avec le niveau d'éducation qui est plus étudié et rapporté dans les autres études à ce sujet. Nous pouvons par extension rapporter le fait qu'être ouvrier et/ou employé est associé à un niveau d'étude plus bas que les cadres ou professions intermédiaires et donc que la propension à exprimer des attitudes défavorables à l'égard des vaccins diminue à mesure que le niveau d'instruction augmente. En effet, $68 \%$ des cadres et à moindre degrés les professions intermédiaires ont un diplôme supérieur à un bac +2 en comparaison de $2 \%$ et $8 \%$ des ouvriers et employés (40). La littérature rapporte que l'éducation supérieure peut être associée à des niveaux d'acceptation du vaccin à la fois plus faibles (13) et plus élevés (1). Ces données mitigées ne peuvent être prise isolément car de multiples influences entrent en jeu (30)(7). Cependant, en tant que déterminant social de la santé, l'éducation est un facteur qui n'a qu'un sens : plus d'éducation entraîne de meilleurs résultats en matière de santé (41).

Une autre caractéristique socio démographique était associée à l'HV dans notre échantillon, celle d'être un parent de fille plutôt que de garçon $(p=0,0027)$. Ce résultat montre le probable impact de la réticence face au vaccin contre le papillomavirus (HPV) où $14 \%$ des parents hésitants ont retardé l'acceptation et $10 \%$ ont refusé la vaccination pour leurs filles comme le démontre l'étude suédoise de Byström et al. qui retrouve une plus grande proportion de parents de filles et d'enfants âgés de 11 à 15 ans dans le groupe « refuseurs sélectif » ce qui pouvait refléter la différence d'acceptation du vaccin anti-HPV (42). Le vaccin anti-HPV ne figure pas sur la liste des vaccins obligatoires (32), ce fait peut conduire les parents à penser à tort que la menace d'infection par le HPV est moindre que celle d'autres maladies et que la vaccination contre le HPV n'est pas nécessaire. 
Cette hésitation est en partie due à la crainte de ses effets secondaires (43). En France, lors des récentes controverses sur le vaccin anti-papillomavirus, certains médecins ont même avancé des arguments contre son utilisation (44).

Dans notre étude, la majorité des hésitants vaccinaux faisait appel à la pratique de médecines alternatives (28\% versus 15\%). Les médecines douces ou alternatives comme l'homéopathie, la naturopathie ont également toujours fait partie du mouvement anti-vaccinal. Dans le climat sociétal actuel, les comportements éco responsables sont promus (45). Ces circonstances amènent à une certaine responsabilisation des choix de consommation. La vaccination rentre dans ce cadre, les patients cherchent à s'autonomiser dans leurs comportements de santé. Plusieurs études montrent une corrélation entre l'HV et l'adoption de médecines alternatives (35)(46).

Ce résultat est conforme à ceux de Verger et al.qui ont observé de plus fortes proportions de médecins pratiquant des médecines alternatives parmi les médecins les plus réticents à la vaccination (47). De même que les utilisateurs de médecines complémentaires et alternatives ont refusé la vaccination plus fréquemment que les autres $(\mathrm{p}<0,001)$ dans l'étude de Zuzak et al., 2008 (45). La population de parents dont les enfants sont atteints de DA est également très sensible à ces pratiques $(38 \%, \mathrm{p}<0,001)$, et cela est largement reconnue (48). Les raisons évoquées par les parents sont de trouver un remède durable sans la peur d'effets secondaires. Ils se tournent donc vers des thérapeutiques dites plus «naturelles » au prix d'une sécurité et efficacité douteuse (49) et parfois grave (50).

La perception de la vaccination par un individu est complexe, elle repose non seulement sur des preuves et des faits scientifiques, mais aussi sur le contexte en termes de politiques économiques, ainsi que sur des facteurs psychologiques et des déterminants sociaux (7)(51).

Les déterminants de l'HV les plus souvent avancés sont semblables dans les deux populations et sont essentiellement ceux concernant la sécurité des vaccins, ce qu'avait également trouvé l'étude de Larson et al.(10), l'absence d'utilité du produit et la présence d'informations négatives relayées par les réseaux sociaux où la course au scoop a également créé un environnement fertile aux idées anti-vaccins (22). Ces facteurs sont des mesures différentes de la perception qu'ont les parents de la vaccination, et ont également été identifiées comme facteurs déterminants de la décision de vaccination dans de nombreuses études (42)(34)(11). 
Il n'est pas surprenant que les problèmes de sécurité soient la principale raison invoquée pour avoir hésité et/ou refusé la vaccination, confirmant ainsi d'autres constatations figurant dans le la littérature (46)(42).

La crainte des maladies à prévention vaccinale a été peu à peu supplantée par la crainte des effets adverses des vaccins et la balance bénéfice-risque s'est totalement inversée dans la société. Au niveau mondial, les risques et les avantages, y compris les problèmes de sécurité des vaccins et les effets indésirables ont été identifiés comme les principales raisons de l'HV des pays ayant répondu à cette section du rapport annuel de l'OMS (52). La sécurité a été également la préoccupation la plus souvent citée comme motif d'hésitation dans les études menées en Europe (11).

Prendre la décision de faire vacciner son enfant résulte de la balance entre la perception du risque des maladies et celle du risque de la vaccination. La perception du risque est cognitive mais également affective et émotionnelle, et cette appréciation subjective semble avoir un impact plus important sur la prise de décision que la connaissance réelle et scientifique du risque $(51)$.

Selon l'étude de Nugier et al., les arguments religieux, fréquents sur les sites anglo-saxons, sont peu présents en France, ce que confirme notre enquête (53).

Les parents ont déclaré que les vaccins les plus soumis à l'HV étaient ceux contre l'hépatite B (VHB) et la grippe. Notre étude met très largement en avant le doute des parents concernant le vaccin contre le VHB avec un parent sur 5 ayant retardé l'acceptation ou refusé, avec un lien pour les affections neurologiques auto-immunes.

Notre enquête montre bien l'impact des polémiques dans notre pays au cours des deux dernières décennies : celle de l'hépatite B en 1998, bien qu'aucune étude n'ait montré de relation causale entre cette vaccination et des maladies neurologiques démyélinisantes (54). Celle de la grippe A(H1N1) en 2009 (1) et celles des infections à papillomavirus humains (HPV) en 2013, conduisant parfois à de réelles crises sanitaires et médiatiques, où l'innocuité des vaccins est mise en doute par la population contribuant à augmenter le refus et l'hésitation des parents. Par exemple, en France, l'intention de refuser la vaccination contre la grippe pandémique est passée de $45 \%$ à $78 \%$ au cours des quatre derniers mois de 2009 (55). 
En revanche, le vaccin ayant eu la meilleure acceptabilité est celui contre le DTP, seul vaccin obligatoire avant la loi du 1 er janvier 2018, mais recommandé qu'à partir de 6 ans, ce qui prouve que les parents ne savent pas forcément les valences présentes dans les vaccins, et de la nécessité d'élargir le nombre de vaccins obligatoires (56).

Deux tiers des parents (67\%) déclarent être aujourd'hui favorables à cette extension des 11 vaccinations obligatoires, parmi lesquels 10\% (selon leur souvenir) ne l'étaient pas en décembre 2017. Les efforts d'informations doivent continuer, puisqu'en dépit de la communication mise en œuvre autour de la mesure près de la moitié des parents indiquent ne pas savoir encore ce qu'elle recouvre et un tiers s'estime mal informé sur ces obligations (57). Ces impressions positives se font également ressentir chez les professionnels de santé, une majorité (69\%) estime que l'obligation s'est accompagnée d'une diminution de la réticence des parents vis-à-vis de la vaccination (57).

Ces vaccins pourraient également être perçus par les parents comme étant contre des maladies "bénignes", par rapport aux vaccins contre des maladies perçues comme "graves" et mettant la vie en danger. La disparition des grandes pandémies rend moins visible les effets positifs des vaccins. La perception du risque occupe une place centrale en matière de comportement santé comme le confirme la méta analyse de Brewer et al.(58). Dans notre étude nous mettons en lien le fait de penser que les vaccins protègent des maladies graves et le moindre risque d'être hésitant vaccinal ( $\mathrm{p}=0,001)$, ce que démontre l'étude de Dubé et al. menée au Qubec (Canada) où les personnes ayant accepté les vaccins présentaient la plus grande proportion de gravité perçue (34). Il est donc urgent de rétablir la confiance des parents dans le vaccin.

La population de parents d'enfants atteints de DA était comparable à la population globale hormis sur les caractéristiques des enfants. Ils étaient pour la plupart âgés de plus de deux ans, or on sait que la DA est une maladie qui se développe vers le sixième mois de vie en moyenne (19), notre étude se déroulait en milieux hospitalo universitaire donc plus à même de recenser des cas graves de dermatologie pédiatrique et donc des DA résistantes aux traitements de première intention et trainante dans le temps avec des enfants plus âgés (59). 
En effet nous retrouvions chez plus d'un quart des enfants atteints de DA d'autres maladies atopiques associées ce qui suggèrent des patients plus compliqués à prendre en charge, étant donné le lien présumé entre la gravité de la DA et le développement de comorbidités atopiques notamment pour l'asthme (15). Comme le montrent les résultats d'une enquête nationale menée aux États-Unis : la prévalence de l'asthme était de 36,9\% chez les patients avec DA sévère alors qu'elle n'était que de $24,3 \%$ en cas de DA légère à modérée $(p=0,02)(60)$.

La même proportion de ces maladies atopiques était retrouvée dans les autres études : 30\% d'asthme, $26 \%$ d'allergie alimentaire, $19 \%$ rhinite allergique et $12 \%$ de conjonctivite allergique ce qui fournit la base du concept de « marche atopique » (15).

Les garçons étaient plus représentés dans la population DA dans notre étude $(p=0,0012)$. Le sex ratio est variable selon les études, on retrouve plus de garçons également dans la série de Kay et al. (19).

Les facteurs génétiques dans les manifestations de la DA ne font aucun doute, en effet on retrouve $22 \%$ ayant un frère ou une sœur atteinte de DA également. Les études familiales retrouvent un diagnostic d'eczéma atopique plus fréquent pour ceux dont le frère ou la sœur souffrent également d'eczéma atopique que pour ceux qui n'en souffraient pas $(36 \%$ versus $24 \%, p=0,05)(20)$. Ces facteurs font même partis des critères diagnostiques simplifiés définis par le groupe UK Working Party's (61).

Le désir des parents de trouver une cause externe à l'état de leur enfant peut entraîner une focalisation sur l'allergie et expliquer probablement aussi la recherche acharnée de cette cause "externe" (22)(20). Dans notre étude, les déterminants de l'HV dans la population DA se distinguaient de la population globale sur une part plus importante de « mauvaise expérience, réaction dans le passé avec le vaccin » dans la question à choix multiple. Les vaccins sont souvent incriminés par les familles, même si plusieurs études en démontrent le contraire (62). Les programmes de vaccination n'expliquent pas la prévalence croissante des maladies atopiques, et il est rare que des enfants développent une réaction grave à un vaccin $(62)(21)$. Toutefois, les risques de ne pas vacciner les enfants dépassent de loin le risque d'allergie. Les parents doivent être encouragés à ne pas retarder la vaccination efficace de leurs enfants parce qu'ils soupçonnent que les vaccins pourraient favoriser les allergies au cours des premières années de la vie. 
Le nombre de consultations pour une DA chez le moins de 18 ans représentait plus d'un tiers des activités du secteur de dermatologie pédiatrique, ce qui suggère un impact socioéconomique non négligeable (18). Ce chiffre de consultations pour DA est important car notre service regroupe un programme d'ETP avec une file active d'une centaine de patients sur ces dernières années.

Une étude épidémiologique suisse retrouvait une fréquence de consultation pour DA en dermatologie similaire ( $26 \%$ chez les moins de 16 ans) avec plus de trois quarts des patients adressés par les pédiatres. La DA constituait le motif de consultation le plus fréquent dans toutes les tranches d'âge (59).

Malgré des recommandations et les stratégies de prise en charge thérapeutique (61), les pratiques diffèrent encore fortement. La discordance des discours entre les soignants, les nombreuses croyances populaires autour de cette maladie et la corticophobie des malades et des soignants rendent la prise en charge difficile (63).

Nous avons montré que les parents d'enfants atteints de DA avaient plus de craintes ou réticences envers les dermocorticoïdes que ceux dont l'enfant avait une autre pathologie dermatologique $(54 \%$ versus $22 \%, \mathrm{p}<0,001)$. Cela a été évalué par la simple question binaire : "Avez-vous des craintes ou réticences concernant les crèmes à base de cortisone? 》. Cela constitue une force de notre étude car ce lien est également retrouvé dans des études antérieures réalisées sur de plus gros effectifs (28)(29)(64).

Notre étude est à notre connaissance la première à établir, chez les familles d'enfants atteints de DA un lien entre le fait d'être corticophobe et hésitant vaccinal selon la définition de l'OMS. Ce lien a été réalisé grâce au score TOPICOP (28)(29). C'est un score multidimensionnel, un outil pratique et efficace pour dépister et prendre en charge la corticophobie au quotidien tant chez les professionnels de santé que chez les parents d'enfants atteints de DA (63). Nous avons choisi de le généraliser initialement à l'ensemble des parents d'enfants suivis en dermatologie, comme ce fut le cas dans l'étude de Moawad et al. pour les parents d'enfants atteints de psoriasis (64), mais le nombre de données manquantes chez les parents d'enfants souffrant d'une autre pathologie dermatologique était trop élevé pour que l'analyse soit comparative. Ils ne devaient probablement pas se sentir concernés par le sujet. 
Il n'y a pas de seuil défini de corticophobie avec ce score, la récente étude internationale a publié un score moyen mondial de 44,7\%+/- 20,5\% et l'étude nationale de 2013 avait retrouvé un score de 43,9\%+/-19,6\%, chez les patients atteints de DA.

Nous avons retrouvé dans notre étude un score TOPICOP global de corticophobie plus faible que ceux retrouvés dans ces deux études (28)(29), cela est peut-être dû à une population de parents d'enfants suivis à la fois à l'hôpital et en ville et donc peut-être moins grave et moins informée que la nôtre. Toutefois la dimension évaluant les peurs des crèmes à base de cortisone était similaire $(47 \%$ versus $46,4 \%)$. Il peut y avoir plusieurs raisons à cela : expérience personnelle, conseils divergents des pharmaciens, médecins, et amis, les informations trouvées sur Internet et dans la presse (50)(25)(65). Les origines de ces craintes sont rarement explorées (26). Il semblerait que ce phénomène soit plus lié à une désinformation plutôt qu'à une irrationalité. Il s'ensuivrait donc que l'éducation ciblée des patients, des parents et des médecins pourrait diminuer la phobie et améliorer l'adhésion au traitement et ainsi diminuer les échecs thérapeutiques dans la DA (65). 


\section{3) Biais et limites de l'étude}

Cette étude a quelques limites. Premièrement, les résultats peuvent être biaisés en raison de la petite taille de l'échantillon et ne peuvent donc pas refléter les caractéristiques de l'ensemble de la population.

Les personnes échantillonnées appartenaient géographiquement à la région Provence Alpes Côte d'Azur (PACA) ce qui a pu surestimer l'hésitation dans notre population par rapport à la population française, dans la mesure où l'on note dans cette région une plus faible adhésion vaccinale corrélée à une moins bonne couverture vaccinale. En effet seulement $72,1 \%$ des habitants de la région se déclarent favorables (plutôt ou très) à la vaccination contre $76 \%$ en France métropolitaine avec une hésitation plus marquée que celle retrouvée dans notre étude estimée à 48,9\% selon la définition de l'OMS (66). Cela peut constituer une limite de notre étude puisque non représentative de l'opinion globale des Français.

Le caractère déclaratif des données recueillies sans vérification du carnet de santé a pu sur ou sous-estimer le statut vaccinal en raison d'un biais de mémoire et/ou de désirabilité sociale. En effet dans une enquête menée du baromètre de 2016 en région PACA, les parents d'enfants de 1 à 15 ans déclarent avoir fait vacciner leurs enfants à hauteur de $90 \%$ pour le vaccin ROR et de seulement $38 \%$ pour le vaccin contre l'hépatite $\mathrm{B}$. Ces chiffres sont bien inférieurs à ceux de la couverture vaccinale au niveau national chez ces jeunes enfants (36)(66). Cependant la confidentialité des données recueillies a quelque peu atténué le biais de désirabilité sociale.

Cette question du statut vaccinal peut traduire une certaine confusion dans les notions de vaccins « obligatoires» et « recommandés ». La coexistence dans le calendrier vaccinal d'obligations et de recommandations vaccinales peut-être source de confusion malgré notre rappel dans le questionnaire. Les vaccins recommandés, pourtant prioritaires, sont parfois considérés comme facultatifs. Selon une étude de l'INPES en 2004, 53 \% des patients pensent que les vaccins recommandés sont moins nécessaires que les vaccins obligatoires (67). 
Dans notre étude, la parité n'était pas respectée, les mères consultaient plus souvent que les pères pour leurs enfants ( $78 \%$ versus $22 \%)$, cependant elles n'étaient pas plus hésitantes. Ce résultat était comparable à l'étude italienne de Giambi et al. qui retrouvait près de $73 \%$ de mères répondantes (46). Cela pourrait refléter l'attention accrue accordée aux questions liées à la vaccination par les mères, qui prennent plus souvent les décisions concernant la santé de leur enfant (68). Nous doutons cependant que cela exprime un biais car nous sommes convaincus que, bien que les mères emmènent généralement l'enfant se faire vacciner, la décision est prise par les deux parents.

Le recueil des données s'est constitué sur les différentes activités du service de dermatologie permettant un plus grand nombre de participations et une meilleure représentativité. En revanche il portait uniquement sur un seul centre. Les parents qui étaient interrogés au sein de la structure, avaient été précédemment adressés par un autre médecin (hospitalier ou non) ce qui a pu engendrer un biais en estimant que ces patients-là étaient plus graves (maladie chronique) ou avaient déjà été sensibilisés par diverses intervenants et informations, et donc peut-être mieux vaccinés que la population générale. En effet notre hôpital étant un centre hospitalo-universitaire, il regroupe une fréquence plus élevée de visites d'enfants souffrant de troubles cutanés chroniques qui sont peut-etre mieux informés car notre centre dispose également d'une école de l'atopie (59).

L'utilisation d'un questionnaire écrit uniquement en Français et qui devait être entièrement rempli par les participants eux-mêmes a également déterminé un autre biais : celui de l'alphabétisation. Il excluait la participation des nouveaux immigrants, qui peuvent également être sous-représentés et avoir des attitudes différentes à l'égard des vaccinations (52). Cette question a été identifiée dans des études précédentes comme un obstacle à l'accès aux soins de santé et un facteur qui contribue à ce qu'ils perçoivent les établissements de santé comme une menace, ce qui entrave leur l'observance des traitements (41). 


\section{4) Propositions d'amélioration}

« La façon d'informer est aussi importante que l'information elle-même » (69).

Pour Holt, nous sommes à l'ère de la " post confiance » et une information issue des autorités ne doit plus être délivrée sur un mode paternaliste descendant mais bien être adaptée aux demandes de prise de décision des individus qui sont informés (70).

L'influence considérable des recommandations des prestataires de soins sur l'acceptation des vaccins est bien documentée dans la littérature (46), ce que confirme notre étude avec $72 \%$ de parents qui estiment que les professionnels de santé encouragent la vaccination loin devant les politiques, enseignants et religieux. Benin et al. ont constaté que la confiance était essentiel pour que les nouvelles mères prennent des décisions concernant la vaccination de leurs enfants, compte tenu de leur manque de compréhension des maladies évitables par la vaccination (69).

Les parents hésitants aux vaccins constituent un groupe beaucoup plus large que ceux qui refusent complètement les vaccins et sont potentiellement plus aptes à accepter des changements de comportement (38).

D’une façon générale, l'ensemble du corps médical et des soignants doit avoir des réponses concertées et approfondies issues d'une source d'information bien établie. Car si l'information du médecin fait défaut, elle est comblée par des sources non fiables telles que les médias ou les conseils communautaires avec les conséquences qu'on leurs connaît. La santé en France est le troisième recours à internet, sachant qu'un Français sur deux utilise internet pour s'informer sur un sujet de santé (22). Les médias devraient donc être mieux utilisés et servir à la diffusion d'une information de qualité car bien souvent ressentie comme délétère à la relation médecin/patient et responsable d'une discréditation de la parole médicale. L'étude de Nugier et al. montre que, lors d'une recherche « neutre » ou « orientée» sur la vaccination, entre 11 et 24 $\%$ des sites suggérés par Google France sont des sites anti-vaccinaux (53).

Pour y pallier, certains réseaux comme Facebook, Amazon et Youtube ont d'ailleurs haussé le ton contre les contenus « antivax » en supprimant les groupes anti-vaccins de leur moteur de recherche (71).

Il a également été prouvé dans une étude réalisée aux États-Unis et en Australie, l'importance de blogs de parents et de groupes de discussion pro-vaccins qui poussent à un changement de politique plutôt qu'à une confrontation publique (72). 
Utiliser ces médias pour diffuser une information de qualité est possible et certainement une des clefs pour répondre à cette défiance.

Ces méthodes de communication et les connaissances à transmettre aux patients devraient faire l'objet d'une formation universitaire et continue en vaccinologie des professionnels de santé. La vaccinologie a longtemps été méconnue et un retard a été pris dans la formation des médecins comme des professionnels de santé amenés à vacciner ou à conseiller dans ce domaine, les rendant vulnérables face aux parents (12). Une étude démontre ce manque de formation des jeunes médecins puisque $66 \%$ et $90 \%$ des étudiants de dernière année du 2 ème cycle d'études de médecine se sentaient insuffisamment préparés pour faire face aux questions sur les potentiels effets indésirables des vaccins (73). Cela pourrait être à l'occasion de la réforme des $1^{\text {er }}$ et $2^{\text {ème }}$ cycle des études médicales ou encore via la stratégie nationale de santé, d'instaurer pour les étudiants en santé un stage de trois mois dans les écoles et entreprises pour y accomplir des missions de prévention, dépistage, et de sensibilisation (36).

Le parcours de soin reste compliqué en France avec de nombreuses étapes chronophages. Les solutions pourraient être : un stock de vaccins chez les médecins, la possibilité de se faire vacciner à la pharmacie directement ou à l'école ? (74)

Impliquer l'école dans le cadre des « parcours éducatifs de santé » pour sensibiliser dès le plus jeune âge est pratiqué dans les pays d'Europe du Nord et au Royaume Uni et obtient des résultats remarquables en multipliant les opportunités de vacciner. Instaurer en France cette vaccination dans les écoles reste un projet ambitieux et risqué à en juger par l'expérience de la vaccination contre l'hépatite B (4).

Il pourrait également être intéressant de développer des stratégies éducatives pour le patient. En médecine libérale où le temps est plus limité, une revue de la littérature démontre l'efficacité de l'entretien motivationnel même pour des consultations durant moins de 20 minutes (75). Il a été testé avec succès dans de nombreux domaines de prévention (alcool, nutrition, activité physique..) (74). Un entretien dédié à la vaccination permettrait via une session didactique d'améliorer la qualité de la communication. 
Il a été démontré précédemment que la multidisciplinarité des équipes et des groupes de soutien mis en place spécifiquement autour de l'éducation et la qualité de vie a réussi à réduire l'anxiété chez les parents touchés par la DA (76).En effet la prise en charge de la DA de l'enfant est partagée entre les médecins généralistes, les pédiatres et les dermatologues en fonction de la gravité de la maladie et de l'âge des enfants. Les problèmes dermatologiques sont si fréquents dans l'enfance que le médecin de premier recours et le pédiatre seront confrontés à un grand nombre d'entre eux. Une formation ciblée et orientée des pédiatres sur cette pathologie et sa thérapeutique, ainsi qu'une meilleure sensibilisation des dermatologues aux vaccins permettrait une prise en charge optimale de l'enfant. Une consultation mixte entre pédiatres et dermatologues pourrait peut-être avoir un intérêt dans le cas de cette maladie chronique (59).

Dans une étude évaluant l'influence de l'éducation des patients sur la corticophobie, une séance d'éducation de 10 à 15 minutes dirigée par un dermatologue avec des instructions écrites a entraîné une diminution de 43,2 \% du score de l'indice de phobie (65).

Des données récentes suggèrent même que les infirmières sont aussi compétentes que les médecins dans la DA pour un coût moindre et une satisfaction des patients supérieure (77).

Les séances d'ETP pourraient en fait constituer d'excellents contextes pour aborder ces peurs avec les familles participantes, améliorant ainsi leurs connaissances sur la sécurité et l'efficacité des dermocorticoïdes et des vaccins, avec une réduction ultérieure et possible de la corticophobie et vaccinophobie. Des recherches plus poussées ainsi que des scores adaptés à cette population seront nécessaires pour mieux comprendre quand, comment et pourquoi ces croyances se forment afin de prévenir l'apparition de cette HV.

Dans un contexte international et français de doutes à l'encontre de la vaccination, notre travail s'inscrit seulement quelques mois avant le début de l'épidémie de Covid-19 qui frappe aujourd'hui le monde entier, et où une course contre la montre s'organise parmi les plus grands laboratoires et groupes pharmaceutiques à la recherche d'un vaccin sûr et efficace. Fera-t-il reprendre conscience aux Français de la gravité de maladies possiblement circulantes et de la réémergence de certaines? 


\section{CONCLUSION}

Au terme de notre travail, nous avons montré pour la première fois que la prévalence de l'HV est plus importante dans les familles d'enfants atteints de DA et qu'elle a pour conséquence inquiétante un retard vaccinal. Nous avons aussi montré que cette catégorie de parents hésitants vaccinaux avait tendance à avoir plus de craintes vis-à-vis des dermocorticoïdes avec un score de corticophobie plus élevé concernant les fausses croyances de ces topiques. Ainsi, il semble que l'adhésion thérapeutique soit un réel problème chez ces parents-là. Les déterminants de cette HV sont cependant similaires avec la population globale de notre échantillon.

Les parents d'enfants atteints de DA ont tendance à établir un lien de cause à effet entre des événements survenant à peu près au même moment dans la vie de leur enfant, comme l'apparition de la dermatite atopique et la primovaccination.

Leurs attitudes et convictions inspirent une certaine méfiance à l'égard de la médecine. Ils constituent un sous-groupe important pour lequel des interventions ciblées de santé publique sont nécessaires. Des travaux supplémentaires sont donc utiles, à la fois pour confirmer les liens entre corticophobie et vaccinophobie et suivre ce phénomène plus global de «défiance médicale » qui, par définition, évolue dans le temps. Une bonne communication afin de convaincre et de rassurer ces familles fait partie d'un ensemble de mesures nécessaires pour maintenir une bonne couverture vaccinale chez l'enfant et une prise en charge optimale de leur DA. 


\section{BIBLIOGRAPHIE}

1. Peretti-Watel P, Verger P, Raude J, Constant A, Gautier A, Jestin C, et al. Dramatic change in public attitudes towards vaccination during the 2009 influenza A(H1N1) pandemic in France. Euro Surveill. 31 oct 2013;18(44).

2. Plan d'action mondial pour les vaccins.pdf [Internet]. [cité 17 nov 2019]. Disponible sur: https://apps.who.int/iris/bitstream/handle/10665/79315/9789242504989_fre.pdf?sequence=1

3. Darmon P. Les premiers vaccinophobes. Sciences Sociales et Santé. 1984;2(3):127-34.

4. André FE. Vaccinology: past achievements, present roadblocks and future promises. Vaccine. 30 janv 2003;21(7-8):593-5.

5. Baromètre santé 2016 [Internet]. [cité 24 déc 2019]. Disponible sur: /etudes-etenquetes/barometres-de-sante-publique-france/barometre-sante-2016

6. Report working group vaccine heitancy final [Internet]. [cité 2 nov 2019]. Disponible sur: https://www.who.int/immunization/sage/meetings/2014/october/1_Report_WORKING_GROUP_vacc ine_hesitancy_final.pdf

7. MacDonald NE, SAGE Working Group on Vaccine Hesitancy. Vaccine hesitancy: Definition, scope and determinants. Vaccine. 14 août 2015;33(34):4161-4.

8. Les cas de rougeole ont bondi de $300 \%$ dans le monde au premier trimestre 2019. Le Monde.fr [Internet]. 15 avr 2019 [cité 25 mars 2020]; Disponible sur:

https://www.lemonde.fr/societe/article/2019/04/15/les-cas-de-rougeole-ont-bondi-de-300-dans-lemonde-au-premier-trimestre-2019_5450513_3224.html

9. Dans toute l'Europe, des flambées épidémiques compromettent l'élimination de la rougeole [Internet]. 2017 [cité 24 déc 2019]. Disponible sur: http://www.euro.who.int/fr/mediacentre/sections/press-releases/2017/measles-outbreaks-across-europe-threaten-progress-towardselimination

10. Larson HJ, de Figueiredo A, Xiahong Z, Schulz WS, Verger P, Johnston IG, et al. The State of Vaccine Confidence 2016: Global Insights Through a 67-Country Survey. EBioMedicine. 13 sept 2016;12:295-301.

11. Yaqub O, Castle-Clarke S, Sevdalis N, Chataway J. Attitudes to vaccination: a critical review. Soc Sci Med. juill 2014;112:1-11.

12. Verger P, Fressard L, Collange F, Gautier A, Jestin C, Launay O, et al. Vaccine Hesitancy Among General Practitioners and Its Determinants During Controversies: A National Cross-sectional Survey in France. EBioMedicine. août 2015;2(8):891-7.

13. Rey D, Fressard L, Cortaredona S, Bocquier A, Gautier A, Peretti-Watel P, et al. Vaccine hesitancy in the French population in 2016, and its association with vaccine uptake and perceived vaccine risk-benefit balance. Euro Surveill [Internet]. 26 avr 2018 [cité 3 nov 2019];23(17).

Disponible sur: https://www.ncbi.nlm.nih.gov/pmc/articles/PMC5930729/

14. Asher MI, Montefort S, Björkstén B, Lai CK, Strachan DP, Weiland SK, et al. Worldwide time trends in the prevalence of symptoms of asthma, allergic rhinoconjunctivitis, and eczema in childhood: ISAAC Phases One and Three repeat multicountry cross-sectional surveys. The Lancet. 26 août 2006;368(9537):733-43.

15. Bantz SK, Zhu Z, Zheng T. The Atopic March: Progression from Atopic Dermatitis to Allergic Rhinitis and Asthma. J Clin Cell Immunol [Internet]. avr 2014 [cité 2 avr 2020];5(2). Disponible sur: https://www.ncbi.nlm.nih.gov/pmc/articles/PMC4240310/

16. Fishbein AB, Mueller K, Kruse L, Boor P, Sheldon S, Zee P, et al. Sleep disturbance in children with moderate/severe atopic dermatitis: A case-control study. J Am Acad Dermatol. 2018;78(2):336-41.

17. Beattie PE, Lewis-Jones MS. A comparative study of impairment of quality of life in children with skin disease and children with other chronic childhood diseases. Br J Dermatol. juill 2006;155(1):145-51. 
18. Su JC, Kemp AS, Varigos GA, Nolan TM. Atopic eczema: its impact on the family and financial cost. Arch Dis Child. févr 1997;76(2):159-62.

19. Kay J, Gawkrodger DJ, Mortimer MJ, Jaron AG. The prevalence of childhood atopic eczema in a general population. J Am Acad Dermatol. janv 1994;30(1):35-9.

20. Harris JM, Cullinan P, Williams HC, Mills P, Moffat S, White C, et al. Environmental associations with eczema in early life. Br J Dermatol. avr 2001;144(4):795-802.

21. Grüber C, Warner J, Hill D, Bauchau V. Early atopic disease and early childhood immunization - is there a link? Allergy. 2008;63(11):1464-72.

22. Heininger U. An internet-based survey on parental attitudes towards immunization. Vaccine. 11 sept 2006;24(37):6351-5.

23. Flohr C, Pascoe D, Williams HC. Atopic dermatitis and the 'hygiene hypothesis': too clean to be true? British Journal of Dermatology. 2005;152(2):202-16.

24. Sulzberger MB, Witten VH. The effect of topically applied compound F in selected dermatoses. J Invest Dermatol. août 1952;19(2):101-2.

25. Li AW, Yin ES, Antaya RJ. Topical Corticosteroid Phobia in Atopic Dermatitis: A Systematic Review. JAMA Dermatol. 01 2017;153(10):1036-42.

26. Fischer G. Compliance problems in paediatric atopic eczema. Australasian Journal of Dermatology. 1996;37(s1):S10-3.

27. pcsese2017-1-Agriculteurs | Insee [Internet]. [cité 22 mars 2020]. Disponible sur: https://www.insee.fr/fr/metadonnees/pcsese2017/categorieSocioprofessionnelleAgregee/1?champRech erche $=$ true

28. Moret L, Anthoine E, Aubert-Wastiaux H, Le Rhun A, Leux C, Mazereeuw-Hautier J, et al. TOPICOPC: a new scale evaluating topical corticosteroid phobia among atopic dermatitis outpatients and their parents. PLoS ONE. 2013;8(10):e76493.

29. Stalder J-F, Aubert H, Anthoine E, Futamura M, Marcoux D, Morren M-A, et al. Topical corticosteroid phobia in atopic dermatitis: International feasibility study of the TOPICOP score. Allergy. nov 2017;72(11):1713-9.

30. Larson HJ, Jarrett C, Eckersberger E, Smith DMD, Paterson P. Understanding vaccine hesitancy around vaccines and vaccination from a global perspective: a systematic review of published literature, 2007-2012. Vaccine. 17 avr 2014;32(19):2150-9.

31. Opel DJ, Taylor JA, Mangione-Smith R, Solomon C, Zhao C, Catz S, et al. Validity and reliability of a survey to identify vaccine-hesitant parents. Vaccine. 2 sept 2011;29(38):6598-605.

32. Tableau des vaccins existants en France [Internet]. [cité 3 avr 2020]. Disponible sur: https://vaccination-info-service.fr/Les-vaccins-existants-en-France/Tableau-des-vaccins-existants-enFrance

33. summary_of_sage_vaccinehesitancy_2pager_French.pdf [Internet]. [cité 3 avr 2020].

Disponible sur:

http://www.who.int/immunization/programmes_systems/summary_of_sage_vaccinehesitancy_2pager French.pdf

34. Dubé E, Gagnon D, Zhou Z, Deceuninck G. Parental Vaccine Hesitancy in Quebec (Canada). PLoS Curr. 7 mars 2016;8.

35. My C, Danchin M, Willaby HW, Pemberton S, Leask J. Parental attitudes, beliefs, behaviours and concerns towards childhood vaccinations in Australia: A national online survey. Aust Fam Physician. mars 2017;46(3):145-51.

36. politique-vaccinale-Tome-1.pdf [Internet]. [cité 26 mars 2020]. Disponible sur: https://www.ccomptes.fr/sites/default/files/2018-01/06-politique-vaccinale-Tome-1.pdf

37. Gust DA, Strine TW, Maurice E, Smith P, Yusuf H, Wilkinson M, et al. Underimmunization among children: effects of vaccine safety concerns on immunization status. Pediatrics. juill 2004;114(1):e16-22.

38. Enkel SL, Attwell K, Snelling TL, Christian HE. « Hesitant compliers »: Qualitative analysis of concerned fully-vaccinating parents. Vaccine. 22 2018;36(44):6459-63.

39. Catégorie socioprofessionnelle selon le sexe et l'âge | Insee [Internet]. [cité 22 mars 2020]. Disponible sur: https://www.insee.fr/fr/statistiques/2489546 
40. Le niveau de diplôme des catégories sociales [Internet]. Observatoire des inégalités. [cité 28 mars 2020]. Disponible sur: https://www.inegalites.fr/Le-niveau-de-diplome-des-categoriessociales?id_theme $=20$

41. Marmot M, World Health Organization, UCL Institute of Health Equity, éditeurs. Review of social determinants and the health divide in the WHO European Region: final report. Copenhagen: World Health Organization, Regional Office for Europe; 2014. 188 p.

42. Byström E, Lindstrand A, Bergström J, Riesbeck K, Roth A. Confidence in the National Immunization Program among parents in Sweden 2016 - A cross-sectional survey. Vaccine. 11 févr 2020 ;

43. Darden PM, Thompson DM, Roberts JR, Hale JJ, Pope C, Naifeh M, et al. Reasons for not vaccinating adolescents: National Immunization Survey of Teens, 2008-2010. Pediatrics. avr 2013;131(4):645-51.

44. Collange F, Fressard L, Pulcini C, Sebbah R, Peretti-Watel P, Verger P. General practitioners' attitudes and behaviors toward HPV vaccination: A French national survey. Vaccine. 3 févr 2016;34(6):762-8.

45. Zuzak TJ, Zuzak-Siegrist I, Rist L, Staubli G, Simoes-Wüst AP. Attitudes towards vaccination: users of complementary and alternative medicine versus non-users. Swiss Med Wkly. 29 nov 2008;138(47-48):713-8.

46. Giambi C, Fabiani M, D’Ancona F, Ferrara L, Fiacchini D, Gallo T, et al. Parental vaccine hesitancy in Italy - Results from a national survey. Vaccine. 01 2018;36(6):779-87.

47. Verger P, Collange F, Fressard L, Bocquier A, Gautier A, Pulcini C, et al. Prevalence and correlates of vaccine hesitancy among general practitioners: a cross-sectional telephone survey in France, April to July 2014. Euro Surveill. 24 nov 2016;21(47).

48. Hughes R, Ward D, Tobin AM, Keegan K, Kirby B. The use of alternative medicine in pediatric patients with atopic dermatitis. Pediatr Dermatol. avr 2007;24(2):118-20.

49. Cheng H-M, Chiang L-C, Jan Y-M, Chen G-W, Li T-C. The efficacy and safety of a Chinese herbal product (Xiao-Feng-San) for the treatment of refractory atopic dermatitis: a randomized, double-blind, placebo-controlled trial. Int Arch Allergy Immunol. 2011;155(2):141-8.

50. Smith SD, Stephens AM, Werren JC, Fischer GO. Treatment failure in atopic dermatitis as a result of parental health belief. Med J Aust. 7 oct 2013;199(7):467-9.

51. Vaccination and trust: How concerns arise and the role of communication in mitigating crises.

$: 50$.

52. Lane S, MacDonald NE, Marti M, Dumolard L. Vaccine hesitancy around the globe: Analysis of three years of WHO/UNICEF Joint Reporting Form data-2015-2017. Vaccine. 18 2018;36(26):3861-7.

53. Nugier A, Limousi F, Lydié N. Vaccine criticism: Presence and arguments on Frenchspeaking websites. Médecine et Maladies Infectieuses. 1 févr 2018;48(1):37-43.

54. Mikaeloff Y, Caridade G, Rossier M, Suissa S, Tardieu M. Hepatitis B vaccination and the risk of childhood-onset multiple sclerosis. Arch Pediatr Adolesc Med. déc 2007;161(12):1176-82.

55. Chanel O, Luchini S, Massoni S, Vergnaud J-C. Impact of information on intentions to vaccinate in a potential epidemic: Swine-origin Influenza A (H1N1). Soc Sci Med. janv 2011;72(2):142-8.

56. Cohen R, Gaudelus J, Leboucher B, Stahl J-P, Denis F, Subtil D, et al. Impact of mandatory vaccination extension on infant vaccine coverages: Promising preliminary results. Med Mal Infect. févr 2019;49(1):34-7.

57. dossier_de_presse_-_semaine_europeenne_vaccination.pdf [Internet]. [cité 3 avr 2020]. Disponible sur: $\bar{h}$ ttps://solidarites-sante.gouv.fr/IMG/pdf/190418_-_dossier_de_presse__semaine_europeenne_vaccination.pdf

58. Brewer NT, Chapman GB, Gibbons FX, Gerrard M, McCaul KD, Weinstein ND. Metaanalysis of the relationship between risk perception and health behavior: the example of vaccination. Health Psychol. mars 2007;26(2):136-45.

59. Wenk C, Itin PH. Epidemiology of pediatric dermatology and allergology in the region of Aargau, Switzerland. Pediatr Dermatol. déc 2003;20(6):482-7. 
60. Silverberg JI, Simpson EL. Association between severe eczema in children and multiple comorbid conditions and increased healthcare utilization. Pediatr Allergy Immunol. août 2013;24(5):476-86.

61. Williams HC, Jburney PG, Pembroke AC, Hay RJ. The U.K. Working Party's Diagnostic Criteria for Atopic Dermatitis. III. Independent hospital validation. British Journal of Dermatology. 1994;131(3):406-16.

62. Grüber C, Nilsson L, Björkstén B. Do early childhood immunizations influence the development of atopy and do they cause allergic reactions? Pediatr Allergy Immunol. déc 2001;12(6):296-311.

63. Bos B, Antonescu I, Osinga H, Veenje S, de Jong K, de Vries TW. Corticosteroid phobia (corticophobia) in parents of young children with atopic dermatitis and their health care providers. Pediatr Dermatol. janv 2019;36(1):100-4.

64. Moawad S, Mahé E, Aubert-Wastiaux H, Phan A, Maruani A, Chiaverini C, et al. Topical Corticosteroid Concerns Among Parents of Children with Psoriasis versus Atopic Dermatitis: A French Multicenter Cross-Sectional Study. Am J Clin Dermatol. avr 2018;19(2):261-5.

65. Lee JY, Her Y, Kim CW, Kim SS. Topical Corticosteroid Phobia among Parents of Children with Atopic Eczema in Korea. Ann Dermatol. oct 2015;27(5):499-506.

66. Verger et al. La perception des vaccins barométre de santé 2016 PACA. 2016;12.

67. Étude Canvac sur la perception de la vaccination auprès d'un échantillon représentatif de 957 personnes. Enquête BVA/Inpes, septembre 2004. Étude non publiée.

68. Cresson G. La production familiale de soins et de santé. La prise en compte tardive et inachevée d'une participation essentielle. Recherches familiales. 2006; $\mathrm{N}^{\circ} 3(1): 6-15$.

69. Benin AL, Wisler-Scher DJ, Colson E, Shapiro ED, Holmboe ES. Qualitative analysis of mothers' decision-making about vaccines for infants: the importance of trust. Pediatrics. mai 2006;117(5):1532-41.

70. Holt D, Bouder F, Elemuwa C, Gaedicke G, Khamesipour A, Kisler B, et al. The importance of the patient voice in vaccination and vaccine safety-are we listening? Clin Microbiol Infect. 1 déc 2016;22 Suppl 5:S146-53.

71. Les activistes antivaccins utilisent les réseaux sociaux pour propager leurs idées [Internet]. [cité 11 avr 2020]. Disponible sur: https://www.lemonde.fr/pixels/article/2019/03/29/avec-les-reseauxsociaux-le-mouvement-antivax-etend-sa-viralite_5442819_4408996.html

72. Vanderslott S. Exploring the meaning of pro-vaccine activism across two countries. Soc Sci Med. 2019;222:59-66.

73. Kernéis S, Jacquet C, Bannay A, May T, Launay O, Verger P, et al. Vaccine Education of Medical Students: A Nationwide Cross-sectional Survey. Am J Prev Med. sept 2017;53(3):e97-104. 74. Dutilleul A, Morel J, Schilte C, Launay O. Comment améliorer l'acceptabilité vaccinale (évaluation, pharmacovigilance, communication, santé publique, obligation vaccinale, peurs et croyances). /data/revues/00405957/v74i1/S004059571830252X/ [Internet]. 23 févr 2019 [cité 12 avr 2020]; Disponible sur: https://www.em-consulte.com/en/article/1278021

75. McClure CC, Cataldi JR, O'Leary ST. Vaccine Hesitancy: Where We Are and Where We Are Going. Clin Ther. août 2017;39(8):1550-62.

76. Ersser SJ, Cowdell F, Latter S, Gardiner E, Flohr C, Thompson AR, et al. Psychological and educational interventions for atopic eczema in children. Cochrane Database Syst Rev. 7 janv 2014;(1):CD004054.

77. Schuttelaar MLA, Vermeulen KM, Drukker N, Coenraads PJ. A randomized controlled trial in children with eczema: nurse practitioner vs. dermatologist. Br J Dermatol. janv 2010;162(1):162-70. 


\section{ANNEXES}

\section{1) «Vaccine Hesitancy Survey Questions : Version 1.0»}

Source : Larson HJ, Jarrett C, Schulz WS, Chaudhuri M, Zhou Y, Dube E, et al. Measuring vaccine hesitancy: The development of a survey tool. Vaccine. $2015 ; 33(34): 4165-75$

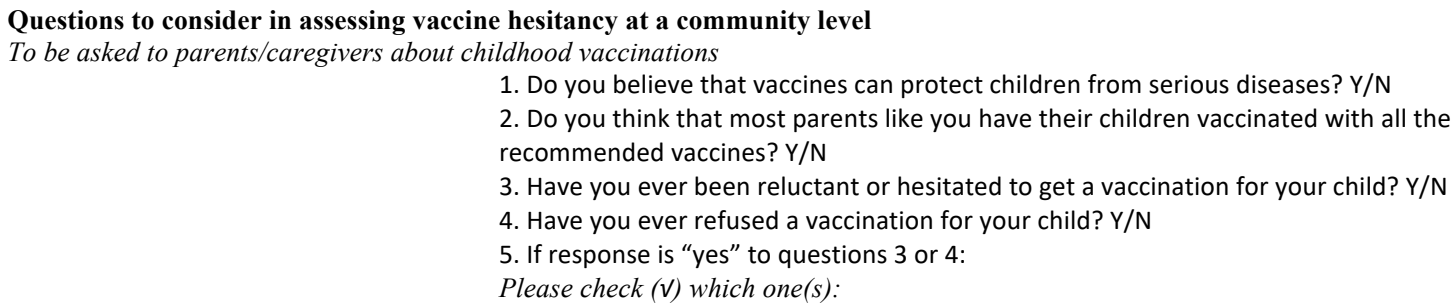

Please check $(v)$ which one (s).

\section{Vaccine names}

Hesitated

Refused

Chickenpox vaccine

Haemophilus influenzae b (Hib) Vaccine

Hepatitis $B$ vaccine

Human papilloma virus (HPV) vaccine

Influenza vaccine

Polio vaccine

Measles vaccine

Meningococcal vaccine

Check ( $v$ if applicable

Mumps vaccine

Rubella vaccine

"Pentavalent" or other combination infant vaccine

Pneumococcal vaccine

Rotavirus vaccine

Tetanus, diphtheria, pertussis vaccine

What was/were the reason(s)? (Use list below to code response)

Reason

Did not think it was needed

Did not know where to get vaccination

Did not know where to get good/reliable information

Heard or read negative media

Did not think the vaccine was effective

Did not think the vaccine was safe/concerned about side effects

Someone else told me that the vaccine was not safe

$\mathrm{Had}$ a bad experience with previous vaccinator/health clinic

Had a bad experience or reaction with previous vaccination

Someone else told me they/their child had a bad reaction

Fear of needles

Not possible to leave other work (at home or other)

Religious reasons

Other beliefs/traditional medicine

Other (please specify):

Leaders : Check $(v)$ if applicable Religious,

Political,
6. Has distance, timing of clinic, time needed to get to clinic or wait at clinic, and/or costs in getting to clinic prevented you from getting your child immunized? $\mathrm{Y} / \mathrm{N}$ If yes, please explain:

7. Are there other pressures in your life that prevent you from getting your child immunized on time? $\mathrm{Y} / \mathrm{N}$

If yes, please specify:

8. Are there any reasons you can think of why children should not be vaccinated? $\mathrm{Y} / \mathrm{N}$

If yes, please specify:

9. Have you ever received or heard negative information about vaccinations? $\mathrm{Y} / \mathrm{N}$

If yes,

a. Please give an example:

ab. Did you still take your child to get vaccinated after you heard the negative information? Y/N

11. Do leaders (religious or political leaders, teachers, health care workers) in your community support vaccines for infants and children? Please indicate below:

Teachers,

Health care worker, Other, please specify 
2) Questionnaire : « La vaccination chez les enfants suivis en dermatologie pédiatrique »

\section{La vaccination chez les enfants suivis pour un eczéma} atopique ou autre maladie de peau

Chers parents,

Je suis interne en dernière année de pédiatrie, je réalise pour ma Thèse, avec l'aide du

Dr S. Mallet et du service de dermatologie de la Timone, une enquête concernant la vaccination afin de comprendre les craintes et réticences des parents d'enfants atteints d'eczéma atopique ou d'une autre maladie de peau. Il est anonyme, individuel et confidentiel. Il a été créé par un groupe de travail de l'Organisation Mondiale de la Santé (OMS) en 2014. Si vous avez besoin de plus de renseignements au sujet des vaccins, merci de contacter le service au 04.91.38.75.95 et de demander Bertille MIANE.

\section{1ère partie : A propos de vous, parent}

1. Quel âge avez-vous ?

$<20$ ans

20-40ans inclus

40-60ans

$>60$ ans

2. Vous êtes :

Un homme

Une femme

3. Quelle est votre profession?

Employé

Ouvrier

Cadre et professions intellectuels supérieurs

Agriculteur, exploitant

Artisan, commerçant et chef d'entreprise

Profession intermédiaire

Retraité

Sans activité professionnelle

Autre, précisez :

4. Quel est votre plus haut niveau d'étude ?

CAP, BEP

Baccalauréat

DEUG, BTS, DUT, DEUST

Licence, licence LMD, Licence professionnelle

Maîtrise

Master, DEA, DESS, diplôme ingénieur

Doctorat, habilitation à diriger des recherches

Autre, précisez : 
Si vous venez avec plusieurs enfants, veuillez répondre pour le plus jeune d'entre eux, merci.

\section{2nde partie : A propos de votre enfant et de sa maladie de peau}

5. Votre enfant $a$ :

0-2 ans (né avant le $1^{\text {er }}$ janvier 2018)

$>2$ ans (né après le $1^{\mathrm{er}}$ janvier 2018) -6 ans

$>6$ ans -12 ans

$>12$ ans -18 ans

6. Quel est le sexe de votre enfant ?

Garçon

Fille

7. De quelle(s) maladie(s) de peau souffre votre enfant ?

Eczéma atopique

Urticaire ou allergie

Autre problème dermatologique

8. S'il est atteint d'eczéma atopique, a-t-il d'autre(s) maladie(s) associée(s) :

Asthme

Allergie alimentaire

Rhinite allergique

Conjonctivite allergique

9. Avez-vous d'autre(s) enfant(s) atteint(s) d'eczéma atopique ?

Oui Non

10. Votre enfant a-t-il déjà participé à des ateliers d'éducation thérapeutique sur l'eczéma atopique ?

Oui Non

11. Avez-vous déjà eu recours pour votre enfant à des médecines alternatives ou

complémentaires (par exemple : homéopathie, naturopathie, phytothérapie, acupuncture,

réflexologie) ?

Oui Non

12. A-t-on déjà prescrit à votre enfant un traitement par crème à base de cortisone (par exemple : diprosone, locapred, betneval..) de plus d'une semaine ?

Oui Non 
13. Avez-vous des craintes ou réticences vis-à-vis des crèmes à base de cortisone ?

Oui Non

Pensez-vous que :

14. Ces crèmes passent dans le sang :

$\square$ pas du tout d'accord $\quad \square$ pas vraiment d'accord $\quad \square$ presque d'accord $\quad \square$ tout à fait d'accord

15. Ces crèmes favorisent les infections

$\square$ pas du tout d'accord $\square$ pas vraiment d'accord $\quad \square$ presque d'accord $\quad \square$ tout à fait d'accord

16. Ces crèmes font grossir :

$\square$ pas du tout d'accord $\square$ pas vraiment d'accord $\square$ presque d'accord $\square$ tout à fait d'accord

17. Ces crèmes abîment la peau :

$\square$ pas du tout d'accord $\square$ pas vraiment d'accord $\square$ presque d'accord $\square$ tout à fait d'accord

18. Ces crèmes ont des effets sur la santé future de votre enfant :

$\square$ pas du tout d'accord $\square$ pas vraiment d'accord $\quad$ presque d'accord $\quad$ tout à fait d'accord

19. Ces crèmes favorisent l'asthme :

$\square$ pas du tout d'accord $\square$ pas vraiment d'accord $\quad \square$ presque d'accord $\quad \square$ tout à fait d'accord

20. Je n'en connais pas les effets secondaires mais j'ai peur de ces crèmes :

$\square$ pas du tout d'accord $\square$ pas vraiment d'accord $\square$ presque d'accord $\square$ tout à fait d'accord

21. J'ai peur d'utiliser une dose de crème trop importante :

$\square$ jamais $\quad \square$ parfois $\quad \square$ souvent $\quad \square$ toujours

22. J'ai peur d'en mettre sur certaines zones où la peau est plus fine comme les paupières :

$\square$ jamais $\square$ parfois $\square$ souvent $\square$ toujours

23. Je traite mon enfant le plus tard possible :

$\square$ jamais $\square$ parfois $\square$ souvent $\square$ toujours

24. Je le traite le moins longtemps possible :

$\square$ jamais $\square$ parfois $\square$ souvent $\square$ toujours

25. J'ai besoin d'être rassuré vis-à-vis du traitement par corticoïde :

$\square$ jamais $\square$ parfois $\square$ souvent $\square$ toujours 


\section{3ème partie : A propos de votre enfant et de ses vaccinations}

26. Quel est le statut vaccinal de votre enfant ? (A l'aide du carnet de santé de votre enfant)

Ses vaccins obligatoires ou non sont à jour

Ses vaccins sont partiellement à jour (il manque au moins une dose pour les vaccins obligatoires (Cf.infra))

Uniquement les vaccins obligatoires

Il n'est pas vacciné

Je ne sais pas

\section{POUR RAPPEL :}

-Les enfants nés à partir du 1er janvier 2018, les vaccinations contre la diphtérie, la poliomyélite, le tétanos, l'Haemophilus $b$, l'hépatite $B$, la coqueluche, la rougeole, les oreillons, la rubéole, le pneumocoque et le méningocoque sont OBLIGATOIRES avant l'âge de 2 ans.

- Pour les enfants nés avant le 1 er janvier 2018, les vaccinations contre la diphtérie, le tétanos et la poliomyélite sont obligatoires aux âges de 2, 4 et 11 mois.

Pour vous aider nous vous rappelons le Calendrier, à découper et à garder ! (;)

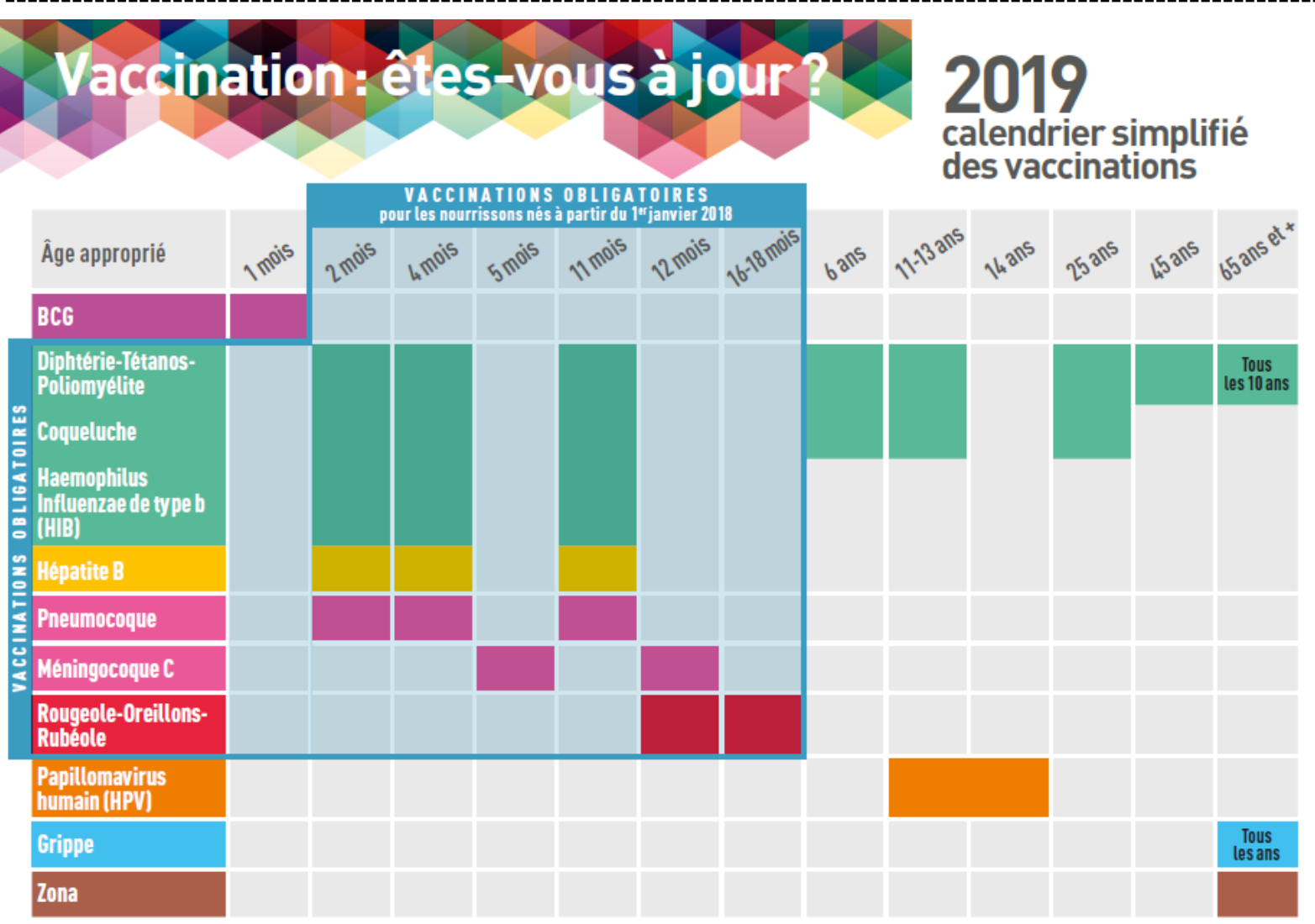


28. Pensez-vous que la majorité des parents vaccine ses enfants selon le calendrier vaccinal, c'est-àdire avec tous les vaccins recommandés?

Oui Non

29. Avez-vous déjà hésité à faire vacciner votre enfant ?

Oui Non

30. Avez-vous déjà refusé de faire vacciner votre enfant ?

Oui Non

31. Si vous avez répondu « oui » aux questions 29 et 30 , pour quel(s) vaccin(s) étai(en)t-ce ?

\begin{tabular}{|l|l|l|}
\hline & $\begin{array}{l}\text { J'ai hésité à ce } \\
\text { vaccin }\end{array}$ & $\begin{array}{l}\text { J'ai refusé à } \\
\text { faire ce vaccin }\end{array}$ \\
\hline BCG (contre la Tuberculose) & & \\
\hline $\begin{array}{l}\text { Diphtérie + Tétanos + Polyomyélite } \\
\text { (REVAXIS) }\end{array}$ & & \\
\hline $\begin{array}{l}\text { Diphtérie + Tétanos + Polyomyélite + Coqueluche } \\
\text { (BOOSTRIXTETRA, INFANRIX TETRA, TETRAVAC- } \\
\text { ACCELLULAIRE, REPEVAX) }\end{array}$ & & \\
\hline $\begin{array}{l}\text { Diphtérie + Tétanos + Polyomyélite + Coqueluche } \\
\text { +Haemophilius influenzae type b } \\
\text { (INFANRIX QUINTA, PENTAVAC) }\end{array}$ & & \\
\hline $\begin{array}{l}\text { Diphtérie + Tétanos + Polyomyélite + Coqueluche } \\
\text { +Haemophilius influenzae type b + Hépatite B } \\
\text { (HEXYON, INFANRIX HEXA, VAXELIS) }\end{array}$ & & \\
\hline $\begin{array}{l}\text { Hépatite B } \\
\text { (ENGERIX B, HBVAXPRO) }\end{array}$ & & \\
\hline $\begin{array}{l}\text { Pneumocoque } \\
\text { (PREVENAR 13, PNEUMO 23, PNEUMOVAX) }\end{array}$ & & \\
\hline $\begin{array}{l}\text { Méningocoque C } \\
\text { (MENJUGATE, MENVEO, NEISVAC, NIMENRIX) }\end{array}$ & & \\
\hline $\begin{array}{l}\text { Rougeole-Oreillons-Rubéole } \\
\text { (M-M-RVAXPRO, PRIORIX) }\end{array}$ & & \\
\hline $\begin{array}{l}\text { Papillomavirus Humain (HPV) } \\
\text { (GARDASIL, CERVARIX) }\end{array}$ & & \\
\hline $\begin{array}{l}\text { Grippe saisonnière } \\
\text { (VAXIGRIP, IMMUGRIP, INFLUVAC, INFLUVAC TETRA) }\end{array}$ & \\
\hline $\begin{array}{l}\text { Méningocoque B } \\
\text { (BEXSERO) }\end{array}$ & & \\
\hline $\begin{array}{l}\text { Gastro entérite à Rotavirus } \\
\text { (ROTARIX, ROTATEQ) }\end{array}$ & \\
\hline $\begin{array}{l}\text { Varicelle } \\
\text { (VARILRIX, VARIVAX) }\end{array}$ & \\
\hline
\end{tabular}


32. Pourquoi avez-vous hésité à faire ce (ou ces) vaccin(s) ou refusé ce (ou ces) vaccins ?

Ce vaccin n'est pas utile

Je ne savais pas où aller pour faire vacciner mes enfants

Je ne savais pas où trouver une information claire et fiable sur ce vaccin

Je ne pouvais pas quitter mon travail (mon domicile ou autre)

Ce vaccin n'est pas efficace

Ce vaccin n'est pas sûr / J'ai peur des effets indésirables

J'ai entendu ou lu des informations négatives à son sujet dans les médias

J'ai eu une mauvaise expérience/réaction avec un vaccin dans le passé

Quelqu'un m'a dit que son enfant a fait une mauvaise réaction à ce vaccin

J'ai eu une mauvaise expérience avec le médecin ou la structure qui m'a vacciné ou vacciné

mes enfants dans le passé

Quelqu'un m'a dit que ce vaccin était dangereux

Raisons religieuses

Autres croyances personnelles

Peur des aiguilles

Autre :

33. La distance, les horaires d'ouverture, le temps d'attente ou le coût du transport pour vous rendre chez votre médecin ou dans un centre de vaccination, vous ont-ils déjà empêché de faire vacciner vos enfants?

Oui Non Si Oui merci de préciser votre réponse :

34. Existe-t-il d'autres raisons dans votre vie qui retardent ou ont retardé la vaccination de vos enfants?

Oui Non Si oui merci de préciser votre réponse :

35. Pensez-vous qu'il y ait d'autres raisons pour lesquelles les enfants ne devraient pas être vaccinés ?

Oui Non Si oui merci de préciser votre réponse :

36. Avez-vous déjà reçu ou entendu des informations négatives au sujet des vaccins ?

Oui Non Si oui merci de donner un exemple :

Avez-vous quand même vacciné vos enfants après avoir reçu cette information ?

Oui Non

37. Les représentants de l'autorité (religieuse, politique, les enseignants et professionnels de santé) dans votre ville encouragent-ils la vaccination des enfants ?

Oui Non

Religieux

Politiques

Enseignants

Professionnels de santé

Autre, précisé : 
Source : Noms commerciaux des vaccins issus du tableau des vaccins actuellement commercialisé présents dans les recommandations vaccinales générales (hors voyages et situations particulières) disponibles en ligne : https://vaccination-info-service.fr/Les-vaccins-existants-en-France/Tableau-desvaccins-existants-en-France

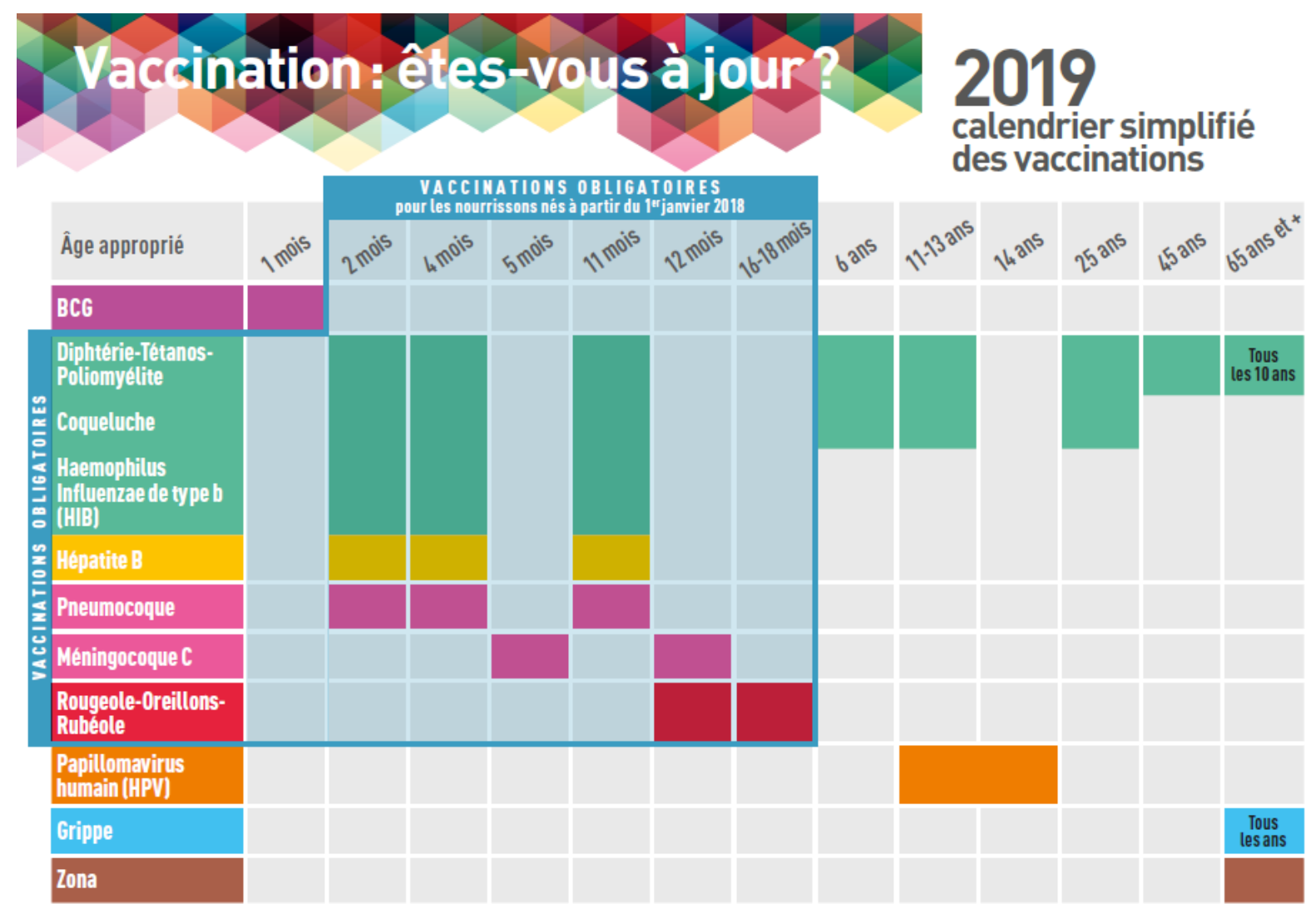




\section{4) Modèle des déterminants de l'hésitation vaccinale mis en place par l'OMS}

Source: World Health organization. Report of the SAGE Working Group on Vaccine Hesitancy. 2014 (issue de l'enquête qualitative sur la vaccination auprès du grand public : Institut français d'OpinionPublique. Etude qualitative sur la vaccination auprès du grand public Rapport d'analyse qualitative. 2016)

\begin{tabular}{|c|c|}
\hline $\begin{array}{l}\text { LES INFLUENCES CONTEXTUELLES: } \\
\text { Influences issues de facteurs historiques, } \\
\text { socio-culturels, environnementaux, } \\
\text { économique ou politique. }\end{array}$ & $\begin{array}{l}\text { a. Communication and médias } \\
\text { b. Rôle d'éventuels leaders influents } \\
\text { c. Les antécédents sanitaires ou controverses non } \\
\text { vaccinales } \\
\text { d. Influences religieuses et culturelles } \\
\text { e. Rôles des politiques vaccinales } \\
\text { f. Obstacles géographiques } \\
\text { g. Rôle de l'industrie pharmaceutique }\end{array}$ \\
\hline $\begin{array}{l}\text { LES INFLUENCES INDIVIDUELLES ET DE } \\
\text { GROUPES: } \\
\text { Influences issues des perceptions } \\
\text { individuelles ou de l'entourage. }\end{array}$ & $\begin{array}{l}\text { a. Antécédents vaccinaux } \\
\text { b. Croyances et attitudes face à la santé et à la } \\
\text { prévention } \\
\text { c. Connaissances et informations } \\
\text { d. Rapport personnel et confiance dans le système } \\
\text { et les professionnels de santé. } \\
\text { e. Rapport bénéfice/risque (perçu, heuristique) } \\
\text { f. L'immunisation comme norme sociale }\end{array}$ \\
\hline $\begin{array}{l}\text { DETERMINANTS LIÉS DE FACON } \\
\text { SPECIFIQUE Á UN VACCIN DONNÉ OU Á LA } \\
\text { VACCINATION : } \\
\text { Influences directement liées à un vaccin ou } \\
\text { au geste vaccinal. }\end{array}$ & $\begin{array}{l}\text { a. Rapport bénéfice/risque (données scientifiques) } \\
\text { b. Introduction d'un nouveau vaccin } \\
\text { c. Mode d'administration } \\
\text { d. Conception des programmes de vaccination et } \\
\text { parcours vaccinal } \\
\text { e. Fiabilité de I'approvisionnement et/ou origine } \\
\text { des vaccins. } \\
\text { f. Calendrier Vaccinal } \\
\text { g. Le coût } \\
\text { h. Le rôle des professionnels de santé }\end{array}$ \\
\hline
\end{tabular}




\section{RESUME}

Introduction : La défiance dans les vaccins et la baisse de la couverture vaccinale qui en découle sont un réel problème de santé publique. Selon notre expérience en dermatologie pédiatrique, ce phénomène d'hésitation vaccinale (HV) défini par le « retard à l'acceptation ou le refus des vaccinations malgré l'existence d'un dispositif d'offre vaccinale », est particulièrement important chez les parents d'enfants atteints de dermatite atopique (DA). Ils y voient, entre autres, un facteur aggravant ou déclenchant de la maladie. L'objectif de notre étude était d'évaluer l'HV auprès des parents d'enfants atteints d'une DA, d'en connaître les déterminants et de rechercher un lien entre vaccinophobie et corticophobie.

Méthode : Nous avons mené une étude observationnelle, unicentrique sur 3 mois auprès des parents d'enfants consultant dans le service de dermatologie de la Timone (Marseille, APHM). Un des 2 parents remplissait un questionnaire anonyme en format papier évaluant 1'HV (Vaccine Hesitancy Survey question) et la corticophobie (score TOPICOP).

Résultats : Sur 312 questionnaires analysés, nous avons mis en évidence un risque plus élevé d'être hésitant vaccinal dans les familles d'enfants atteints de DA ( $52 \%$ versus 38\%, p=0,0021) et ce lien persistait après ajustement $(\mathrm{p}=0,037)$. La conséquence étant un retard vaccinal $(20 \%$ versus $6 \%, \mathrm{p}=0,009)$. Cette $\mathrm{HV}$ était plus marquée si les enfants souffraient d'une autre manifestation atopique comme une rhinite allergique $(p=0,017)$. Dans les familles avec DA, les hésitants avaient tendance à avoir plus de craintes ou réticences à l'application de dermocorticoïdes ( $63 \%$ versus $44 \%, \mathrm{p}=0,053)$, avec un score de corticophobie plus élevé sur les fausses croyances ( $40 \%$ versus $32 \%, \mathrm{p}=0,048)$.

Discussion : Notre étude montre que l'adhésion thérapeutique est un réel problème chez les parents d'enfants atteints de DA. Nous avons montré qu'ils étaient plus vaccinophobes, plus mal vaccinés et qu'il y avait un lien entre vaccinophobie et corticophobie. Les déterminants de cette HV sont similaires. La peur des effets indésirables, la perte de confiance dans la sécurité du produit, la perception d'une balance bénéfice/risque défavorable ainsi que la conviction que les vaccins ne protègent pas de maladies graves sont les principales préoccupations des parents hésitants vaccinaux, relayées en grande partie par les informations négatives des médias.

Conclusion : L'identification des populations à risque d'HV et la compréhension des mécanismes conduisant à une défiance médicale en général est une condition préalable à la conception d'interventions de santé publique.

Mots clés : hésitation vaccinale ; dermatite atopique ; corticophobie ; vaccinophobie ; adhésion thérapeutique 


\section{SERMENT D'HIPPOCRATE}

Au moment d'être admis(e) à exercer la médecine, je promets et je jure d'être fidèle aux lois de l'honneur et de la probité.

Mon premier souci sera de rétablir, de préserver ou de promouvoir la santé dans tous ses éléments, physiques et mentaux, individuels et sociaux.

Je respecterai toutes les personnes, leur autonomie et leur volonté, sans aucune discrimination selon leur état ou leurs convictions. J'interviendrai pour les protéger si elles sont affaiblies, vulnérables ou menacées dans leur intégrité ou leur dignité. Même sous la contrainte, je ne ferai pas usage de mes connaissances contre les lois de I'humanité.

J'informerai les patients des décisions envisagées, de leurs raisons et de leurs conséquences.

Je ne tromperai jamais leur confiance et n'exploiterai pas le pouvoir hérité des circonstances pour forcer les consciences.

Je donnerai mes soins à l'indigent et à quiconque me les demandera. Je ne me laisserai pas influencer par la soif du gain ou la recherche de la gloire.

Admis(e) dans l'intimité des personnes, je tairai les secrets qui me seront confiés. Reçu(e) à l'intérieur des maisons, je respecterai les secrets des foyers et ma conduite ne servira pas à corrompre les mœurs.

Je ferai tout pour soulager les souffrances. Je ne prolongerai pas abusivement les agonies. Je ne provoquerai jamais la mort délibérément.

Je préserverai l'indépendance nécessaire à l'accomplissement de ma mission. Je n'entreprendrai rien qui dépasse mes compétences. Je les entretiendrai et les perfectionnerai pour assurer au mieux les services qui me seront demandés.

J'apporterai mon aide à mes confrères ainsi qu'à leurs familles dans l'adversité.

Que les hommes et mes confrères m'accordent leur estime si je suis fidèle à mes promesses ; que je sois déshonoré(e) et méprisé(e) si j'y manque.

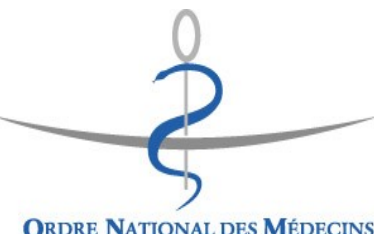


